\title{
A Tale of Two Mysteries in Interstellar Astrophysics: The $2175 \AA$ Extinction Bump and Diffuse Interstellar Bands
}

\author{
F.Y. Xiang ${ }^{1,2}$, Aigen $\mathrm{Li}^{2}$ and J.X. Zhong ${ }^{1,2}$
}

\begin{abstract}
The diffuse interstellar bands (DIBs) are ubiquitous absorption spectral features arising from the tenuous material in the space between stars - the interstellar medium (ISM). Since their first detection nearly nine decades ago, over 400 DIBs have been observed in the visible and near-infrared wavelength range in both the Milky Way and external galaxies, both nearby and distant. However, the identity of the species responsible for these bands remains as one of the most enigmatic mysteries in astrophysics. An equally mysterious interstellar spectral signature is the $2175 \AA$ extinction bump, the strongest absorption feature observed in the ISM. Its carrier also remains unclear since its first detection 46 years ago. Polycyclic aromatic hydrocarbon (PAH) molecules have long been proposed as a candidate for DIBs as their electronic transitions occur in the wavelength range where DIBs are often found. In recent years, the $2175 \AA$ extinction bump is also often attributed to the $\pi-\pi^{*}$ transition in PAHs. If PAHs are indeed responsible for both the $2175 \AA$ extinction feature and DIBs, their strengths may correlate. We perform an extensive literature search for lines of sight for which both the $2175 \AA$ extinction feature and DIBs have been measured. Unfortunately, we found no correlation between the strength of the $2175 \AA$ feature and the equivalent widths of the strongest DIBs. A possible explanation might be that DIBs are produced by small free gas-phase PAH molecules and ions, while the $2175 \AA$ bump is mainly from large PAHs or PAH clusters in condensed phase so that there is no tight correlation between DIBs and the $2175 \AA$ bump.
\end{abstract}

Subject headings: dust, extinction; ISM: lines and bands; ISM: molecules

\footnotetext{
${ }^{1}$ Department of Physics, Xiangtan University, 411105 Xiangtan, Hunan Province, China; fyxiang, jxzhong@xtu.edu.cn

${ }^{2}$ Department of Physics and Astronomy, University of Missouri, Columbia, MO 65211, USA; lia@missouri.edu
} 


\section{Introduction}

The diffuse interstellar bands (DIBs) show up as absorption troughs in the optical/near infrared (IR) wavelength range against the continuous spectra of hot background stars which lie behind diffuse interstellar clouds. The strength of a DIB is often measured in terms of the "equivalent width" (which reflects the wavelength-integrated area of the DIB; see Fig. 1). As already recognized 76 years ago (Merrill et al. 1934), the DIB strengths generally increase with the amount of interstellar material between the background star and the observer, revealing their nature of interstellar origin.

Since their first detection in 1919 by Heger (1922), to date over four hundred DIBs have been observed in both Galactic and extragalactic sources (Snow 2002; Sarre 2006; Hobbs et al. 2008, 2009), including the Large and Small Magellanic Clouds (Ehrenfreund et al. 2002; Cox et al. 2006, 2007a; Welty et al. 2006), M31 (Cordiner et al. 2008b, 2011), M33 (Cordiner et al. 2008a), starburst galaxies (Heckman \& Lehnert 2000), intervening absorption systems toward quasars (Ellison et al. 2008) and damped Ly $\alpha$ absorbers at cosmological distances (DLAs; Junkkarinen et al. 2004, York et al. 2006a, Lawton et al. 2008). It is interesting to note that both the $2175 \AA$ extinction bump (see below) and three weak DIBs at $\lambda 4430 \AA, \lambda 5705 \AA$, and $\lambda 5780 \AA$ have been detected in the quasar intervening DLA system at $z_{\text {abs }}=0.524$ toward AO 0235+164 (Junkkarinen et al. 2004, York et al. 2006a, Lawton et al. 2008).

The $2175 \AA$ extinction bump shows up as the most prominent spectral feature in the interstellar extinction curve - the wavelength dependence of the interstellar dust obscuration (see Fig. 1). It is ubiquitously seen in the Milky Way (see Draine 1989) and has recently also been detected in distant galaxies at redshifts $z>2$ (Elíasdóttir et al. 2009; Noll et al. 2007, 2009; Prochaska et al. 2009; Liang \& Li 2009, 2010). Its detection at $z<2$ has been more frequently reported (e.g. see Motta et al. 2002, Wang et al. 2004, Noll \& Pierini 2005, York et al. 2006b, Inoue et al. 2006, Srianand et al. 2008, Conroy et al. 2010, Zhou et al. 2010, Jiang et al. 2011). The most striking characteristics of the $2175 \AA$ extinction bump are the invariant central wavelength and variable bandwidth: its peak position at $2175 \AA$ is remarkably constant while the bandwidth varies from one line of sight to another (see Whittet 2003).

The exact nature of the carriers of DIBs remains as one of the most enigmatic mysteries in astrophysics (see Sarre 2006), although polycyclic aromatic hydrocarbon (PAH) molecules seem to be a promising candidate (see Crawford et al. 1985, Léger \& d'Hendecourt 1985, van der Zwet \& Allamandola 1985, Salama et al. 1996, 1999, 2011). Other candidate materials such as fullerenes (Ehrenfreund \& Foing 1996) have also received much attention.

The $2175 \AA$ extinction bump also remains unidentified since its first detection over four 
decades ago by Stecher (1965). It was generally attributed to the $\pi-\pi^{*}$ transition in $s p^{2}$ aromatic carbon dust such as graphite (Stecher \& Donn 1965) or PAHs (Joblin et al. 1992, Li \& Draine 2001, Duley 2006).

Are the carriers of DIBs and the $2175 \AA$ extinction bump related? more specifically, are PAHs - a family of various aromatic species which are thought to be widespread in interstellar space as revealed by the ubiquitous "Unidentified IR (UIR)" emission features at 3.3, 6.2, 7.7, 8.6, 11.3 and $12.7 \mu \mathrm{m}$ (Léger \& Puget 1984, Allamandola et al. 1985, Smith et al. 2007, Tielens 2008) - responsible for both DIBs and the $2175 \AA$ extinction bump?

One way to test this hypothesis is to examine whether the strengths of DIBs and the $2175 \AA$ bump are correlated. We have initiated a program to explore the possible correlations between the DIBs and various interstellar extinction properties. In this work we perform a correlation study of the DIB equivalent widths (EWs) and the $2175 \AA$ extinction bump strengths, both are normalized by the interstellar reddening $E(B-V)$. 1 for 84 sightlines of which the DIB equivalent widths, the $2175 \AA$ bump strengths, and $E(B-V)$ are available in the literature. We use the equivalent width (EW) of a DIB to characterize its absorption strength, which measures the area of the DIB absorption profile above the continuum (see the shaded area in the inserted panel in Fig. 1). In $\S 2$ and $\S 3$ we will discuss what would be the most reasonable way to characterize the strength of the $2175 \AA$ extinction bump.

\section{DIBs vs. the $2175 \AA$ Bump: Where Do We Stand?}

Whether the carriers of DIBs are related to the $2175 \AA$ extinction feature has been a topic of extensive research for over three decades. Contradicting conclusions have been drawn in the literature. As summarized in the following paragraphs, this seems to be largely caused by the inconsistencies in determining the $2175 \AA$ bump strength.

Nandy \& Thompson (1975) first speculated that the $2175 \AA$ extinction bump may be correlated with the strength of the $\lambda 4430 \AA$ DIB. They derived the "equivalent widths" of the bump (to represent the bump strengths) of $>60$ sightlines from the interstellar reddening $E(2190 \AA-2500 \AA) \Omega$ They found that the "equivalent widths" of the bump and the central depths of the $\lambda 4430 \AA$ DIB were well correlated, with the correlation coefficient exceeding

\footnotetext{
${ }^{1} E(B-V) \equiv A_{B}-A_{V}=A_{V} / R_{V}$ is the difference between the $B$ band extinction $\left(A_{B}\right)$ and the $V$ band extinction $\left(A_{V}\right)$, where $R_{V} \equiv A_{V} / E(B-V)$ is the total-to-selective extinction ratio.

${ }^{2}$ By assuming a bump width of $360 \AA$ and a mean relationship between $E(2190 \AA-2500 \AA)$ and the central absorption at $2175 \AA$, they took the bump "equivalent width" to be $\approx 160 \AA \mathrm{mag}^{-1} \times E(2190 \AA-2500 \AA)$.
} 
$\sim 0.9$.

Schmidt (1978) compared $E(2200 \AA-3320 \AA)$ with the EWs of the $\lambda 5780 \AA$ DIB in the spectra of 50 early-type stars. He found that they were correlated, although with considerable intrinsic scatter.

Danks (1980) determined the "depths" of the $2175 \AA$ bump from $D_{2175} \equiv A_{2175 \AA}-0.5 \times$ $\left(A_{2500 \AA}+A_{1800 \AA}\right)$ of 30 stars, where $A_{\lambda}$ is the extinction at wavelength $\lambda$. He found a quite tight linear correlation between $D_{2175}$ and the EW of the $\lambda 4430 \AA$ DIB.

Dorschner et al. (1977) estimated the $2175 \AA$ bump strengths of sightlines toward 194 stars from integrating the bump area (with the continuum extinction subtracted) 3 They found a fairly good correlation between the $2175 \AA$ bump strength and the central depth of the $\lambda 4430 \AA \mathrm{DIB}$ (with a correlation coefficient of $\sim 0.74$ ). 4 Similar correlations were found for the $2175 \AA$ bump strength and the EW of the $\lambda 5780 \AA$ DIB (with a correlation coefficient of $\sim 0.81$ ) and that of the $\lambda 5797 \AA$ DIB (with a correlation coefficient of $\sim 0.74$ ).

Wu et al. (1981) estimated the $2175 \AA$ bump strengths from $S \equiv E(2200 \AA-V)-0.5 \times$ $[E(1800 \AA-V)-E(2500 \AA-V)]$ for sightlines toward 110 hot stars. They found that the central depth of the $\lambda 4430 \AA \mathrm{DIB}$ is well correlated with the $2175 \AA$ extinction bump (with a correlation coefficient of $\sim 0.83$ ). Much weaker correlations were found for the $2175 \AA$ bump strength and the EW of the $\lambda 5780 \AA \mathrm{DIB}$ (with a correlation coefficient of $\sim 0.66$ ) and that of the $\lambda 6284 \AA$ DIB (with a correlation coefficient of $\sim 0.63$ ).

Witt et al. (1983) first pointed out that the possible correlation between the $2175 \AA$ bump and the $\lambda 4430 \AA$ DIB suggested by Nandy \& Thompson (1975) and Wu et al. (1981) may merely reflect the fact that both the bump strength and the DIBs correlate with $E(B-V)$. In order to eliminate the common correlation with $E(B-V)$, Witt et al. (1983) compared the central depths of the $\lambda 4430 \AA$ DIB normalized by $E(B-V)$ with the normalized bump strengths $E$ (bump) $/ E(B-V)$ of 20 stars 5 They only found a marginally significant correlation between the normalized strengths of the UV bump and the $\lambda 4430 \AA \mathrm{DIB}$, with a correlation coefficient of $\sim 0.52$. This was confirmed by Seab \& Snow (1984) who explored a larger sample of 50 stars.

\footnotetext{
${ }^{3}$ They approximated the continuum extinction underneath the $2175 \AA$ bump by a straight line between $\lambda^{-1}=3 \mu \mathrm{m}^{-1}$ and $\lambda^{-1}=6 \mu \mathrm{m}^{-1}$.

${ }^{4} \mathrm{Wu}$ et al. (1981) argued that the bump strengths calculated by Dorschner et al. (1977) may probably have included continuous absorption not typically associated with the bump.

${ }^{5}$ Witt et al. (1983) defined $E$ (bump) as the excess extinction at $\lambda^{-1}=4.62 \mu \mathrm{m}^{-1}$ above a linear interpolation of the extinction curve between $\lambda=3.50 \mu \mathrm{m}^{-1}$ and $5.75 \mu \mathrm{m}^{-1}$.
} 
Benvenuti \& Porceddu (1989) compared the EWs of six DIBs ( $\lambda 5780,5797,6196,6203$, 6270 and $6284 \AA$ ) with the $2175 \AA$ bump strengths of a sample of 26 galactic stars, with both quantities normalized by $E(B-V)$. They took the bump height $h_{2175}$ (see Fig. 1 and $\S 3$ ) for the bump strength and found no correlation between the bump and DIBs (even for the closest correlation [for the $2175 \AA$ bump and the $\lambda 6284 \AA$ DIB] the correlation coefficient is only $\sim 0.39)$. Désert et al. (1995) performed a similar analysis for eight DIBs $(\lambda 5707,5780$, $5797,5850,6177,6196,6269,6284 \AA$ ) of 28 stars. In contrary to Benvenuti \& Porceddu (1989), they found that the $2175 \AA$ bump height correlates with the DIB EWs, with the correlation coefficients greater than 0.8 for some DIBs. More recently, Megier et al. (2005) compared the normalized EWs of 11 DIBs with the $2175 \AA$ bump strengths of 49 stars, also using the bump height as a measure of the bump strength. They found that most of the DIBs correlate positively with the extinction bump.

\section{How to Characterize the $2175 \AA$ Extinction Feature Strength?}

In view of the various standards in determining the strength of the $2175 \AA$ bump (see $\S 2)$, an important question one need to address is: what is the most reasonable measure of the strength of the $2175 \AA$ bump? We argue that the area of the continuum-subtracted bump integrated over $\lambda^{-1}$ is an appropriate measure (see below and Fig. 1).

Fitzpatrick \& Massa (1990; hereafter FM90) found that the $2175 \AA$ extinction bump, with the underneath linear background subtracted, can be closely fitted by a Drude profile. The Drude profile, expected for classical damped harmonic oscillators, is characterized by $\lambda_{\mathrm{o}}$ - the peak wavelength, $\gamma$ - the band width, and $c_{3}$ - a parameter relates to the strength of the bump. In the Galactic ISM, the strength and width of the $2175 \AA$ extinction bump vary with environment while its peak position $\lambda_{\mathrm{o}} \approx 2175 \AA$ is quite invariant (see Whittet 2003).

With the extinction curve expressed as $E(\lambda-V) / E(B-V)$, where $E(\lambda-V)$ is the reddening between wavelength $\lambda$ and the visual band $V$, as illustrated in Fig. 1, the area in the $2175 \AA$ bump above the linear background (i.e. the shaded area in Fig. 1) for an extinction curve normalized by $E(B-V)$ is $W_{2175} / E(B-V)=\pi c_{3} / 2 \gamma$ (see Fitzpatrick \& Massa 1986, 1990). The bump height, defined as the maximum height above the linear background, is $h_{2175} / E(B-V)=c_{3} / \gamma^{2}$. We argue that $W_{2175}$ (which is analogous to the concept of equivalent width) is the most reasonable measure of the strength of the $2175 \AA$ extinction bump, particularly in view of the fact that we use the equivalent width of a DIB to characterize its absorption strength, which measures the area of the DIB absorption profile above the continuum (see the shaded area in the inserted panel in Fig. 1). We note that both quantities 
linearly determine the column densities of their respective carriers: as the DIBs are always optically thin, the equivalent width $W_{\text {DIB }}$ of a DIB at wavelength $\lambda$ allows us to determine the column density of its carrier through $N_{\text {DIB }} \approx 1.13 \times 10^{20} \mathrm{~cm}^{-2}\left(W_{\text {DIB }} / \AA\right)(\lambda / \AA)^{-2} f_{\mathrm{d}}^{-1}$, where $f_{\mathrm{d}}$ is the oscillator strength of the transition associated with the DIB (see Herbig 1993). For the $2175 \AA$ extinction bump, the column density of its carrier $N_{\text {bump }}$ is also linearly related to $W_{2175}: N_{\text {bump }}=\left(m_{e} c / \pi e^{2}\right) f_{\mathrm{b}}^{-1} W_{2175}$, where $f_{\mathrm{b}}$ is the oscillator strength (per absorber) associated with the $2175 \AA$ bump 6

The bump strength relates to the bump height through $W_{2175}=(\pi \gamma / 2) h_{2175}$. Since the bump width $\gamma$ varies from one sightline to another, the bump height itself is not sufficient to describe the bump strength.

With $c_{3}$ and $\gamma$ determined from fitting the observed extinction curve with the FM90 decomposition 7 one can obtain the bump strength (normalized by $E(B-V)$ ) from $c_{3}$ and $\gamma$. If $c_{3}$ is unknown, one can still obtain the bump strength simply from the bump height $h_{2175}$ and width $\gamma$.

\section{Correlation Between DIBs and the $2175 \AA$ Extinction Bump}

As a first step in examining whether the $2175 \AA$ extinction bump correlates with the DIBs, we compile a large number of sightlines for which both the interstellar extinction curves and DIBs have been measured (see Tables 1,2 and the Appendix). In this work we focus on nine strong DIBs in the visible wavelength range for which rich sets of high quality spectroscopy data are available: $\lambda 5707 \AA, \lambda 5780 \AA, \lambda 5797 \AA, \lambda 6195 \AA / 6196 \AA, \lambda 6203 \AA$, $\lambda 6269 \AA / 6270 \AA, \lambda 6284 \AA, \lambda 6376 \AA / 6379 \AA$, and $\lambda 6614 \AA$. We group the two DIBs at $\lambda 6376 \AA$ and $\lambda 6379 \AA$ into one band (which is labeled " $\lambda 6376 \AA / 6379 \AA$ ") as they may blend. The DIBs at $\lambda 6195 \AA$ and $\lambda 6196 \AA$ are actually the same DIB, which we refer as the " $\lambda 6195 \AA / 6196 \AA$ ". Similarly, the " $\lambda 6269 \AA / 6270 \AA$ " DIB refers to either the $\lambda 6269 \AA$ DIB or the $\lambda 6270 \AA$ DIB

\footnotetext{
${ }^{6}$ The column density $N_{\text {bump }}$ of the carrier of the $2175 \AA$ extinction bump is linearly proportional to the total area of the bump above the continuum extinction: $N_{\text {bump }} / N_{\mathrm{H}}=1.086\left(m_{e} c / \pi e^{2}\right) f_{\mathrm{b}}^{-1} \int\left(\Delta \tau_{\nu} / N_{\mathrm{H}}\right) d \nu$, where $N_{\mathrm{H}}$ is the hydrogen column density, $m_{e}$ is the electron mass, $c$ is the speed of light, $e$ is the electron charge, and $\Delta \tau_{\nu} / N_{\mathrm{H}}$ is the optical depth (per H column) attributed to the bump (see Draine 1989). Since $\int\left(\Delta \tau_{\nu} / N_{\mathrm{H}}\right) d \nu=(1 / 1.086)\left(\pi c_{3} / 2 \gamma\right) E(B-V) / N_{\mathrm{H}}$ [where $E(B-V) / N_{\mathrm{H}} \approx 1.7 \times 10^{-22} \mathrm{mag} \mathrm{cm}^{2} \mathrm{H}^{-1}$ is the extinction-to-hydrogen ratio in the diffuse ISM], we obtain $N_{\text {bump }}=\left(m_{e} c / \pi e^{2}\right) f_{\mathrm{b}}^{-1} W_{2175}$.

${ }^{7}$ Fizpatrick \& Massa (1990) found that the interstellar extinction curves at $3.3 \mu \mathrm{m}^{-1}<\lambda^{-1}<10 \mu \mathrm{m}^{-1}$ can be decomposed into three components consisting of six parameters - (i) a linear "background" term; (ii) a "Drude profile" term for the $2175 \AA$ extinction feature; and (iii) a far ultraviolet (UV) non-linear rise at $\lambda^{-1}>5.9 \mu \mathrm{m}^{-1}$.
} 
(which are also the same DIB). Unfortunately, the equivalent width information is not always available for the strongest DIB which peaks at $\lambda 4430 \AA$, in the literature most often only the absorption depth has been reported for this DIB.

The DIB EWs and the extinction parameters $\left(c_{3}, \gamma\right)$ are taken from Dorschner et al. (1977), Fitzpatrick \& Massa (1986, 1990), Benvenuti \& Porceddu (1989), Herbig (1993), Jenniskens \& Greenberg (1993), Désert et al. (1995), Sonnentrucker et al. (1997), Thorburn et al. (2003), Galazutdinov et al. (2004), Lewis et al. (2005), Megier et al. (2005), Cox et al. (2007b), Hobbs et al. (2008, 2009), McCall et al. (2010), and Friedman et al. (2011).8 In Table 1 we tabulate for each object the extinction parameters $E(B-V), R_{V}, c_{3}, \gamma$, and $W_{2175} / E(B-V)$, as well as the EWs of the nine DIBs. In the Appendix we elaborate how we obtain the EWs of these DIBs.

In Figure 2 we show the correlation between $W_{\mathrm{DIB}}^{\prime} \equiv W_{\mathrm{DIB}} / E(B-V)$, the normalized DIB EWs, and $W_{2175}^{\prime} \equiv W_{2175} / E(B-V)=\pi c_{3} / 2 \gamma$, the normalized bump strengths.9 It is clearly seen in this figure that the correlation is very poor. To be more quantitative, we calculate the correlation coefficients $r$ of the bump strengths and the DIB EWs, both are normalized to $E(B-V): r=\left\{\left\langle W_{2175}^{\prime} W_{\text {DIB }}^{\prime}\right\rangle-\left\langle W_{2175}^{\prime}\right\rangle\left\langle W_{\text {DIB }}^{\prime}\right\rangle\right\} /\left\{\sigma_{W_{2175}^{\prime}} \sigma_{W_{\text {DIB }}^{\prime}}\right\}$, where $\sigma_{W_{2175}^{\prime}}$ and $\sigma_{W_{\text {DIB }}^{\prime}}$ are the variances of $W_{2175}^{\prime}$ and $W_{\text {DIB }}^{\prime}$, respectively. Table 2 presents the calculated correlation coefficients. We see that $r<0.60$ for all DIBs, indicating that the $2175 \AA$ bump is poorly if not at all correlated with any of the nine DIBs (even for the most "correlated" one, $\lambda 6614 \AA$, the correlation coefficient is only $r \approx 0.58) 10$

\footnotetext{
${ }^{8}$ Thorburn et al. (2003) reported the EWs of nine DIBs at $\lambda 5780 \AA, \lambda 5797 \AA, \lambda 6196 \AA, \lambda 6203 \AA, \lambda 6270 \AA$, $\lambda 6284 \AA, \lambda 6376 \AA, \lambda 6379 \AA$, and $\lambda 6614 \AA$ (in their paper they labeled it $\lambda 6613 \AA$ ). More recently, those authors updated the EWs of five of these DIBs: $\lambda 5780 \AA, \lambda 5797 \AA, \lambda 6196 \AA, \lambda 6284 \AA$, and $\lambda 6614 \AA$ (see Friedman et al. 2011). We therefore adopt the EWs of the four DIBs $(\lambda 6203 \AA, \lambda 6270 \AA, \lambda 6376 \AA$, and $\lambda 6379 \AA)$ of Thorburn et al. (2003), and the EWs of the five DIBs $(\lambda 5780 \AA, \lambda 5797 \AA, \lambda 6196 \AA, \lambda 6284 \AA$, and $\lambda 6614 \AA$ ) of Friedman et al. (2011).

${ }^{9}$ We normalize both $W_{2175}$ and $W_{\text {DIB }}$ by $E(B-V)$ to cancel out the linear correlations of $E(B-V)$ with $W_{\text {DIB }}$ and $W_{2175}$ which reflect the amount of interstellar materials. $W_{\text {DIB }}$ is the EW of a DIB at a specific wavelength.

${ }^{10}$ Although somewhat arbitrary, we feel a close correlation should have $r>0.9$, a good correlation should have $r>0.8$, and a fair correlation should have $r>0.7$. This seems to be in line with previous studies (see $\S 2)$. In studying the correlation between the $2175 \AA$ extinction bump and the far-UV extinction, Greenberg \& Chlewicki (1983) described the correlation with $r=0.731$ as being "poor".
} 


\section{Discussion}

A novel aspect of the present study is that, we measure the bump strength as the area above the linear background (see Fig. 1) instead of the height. Since the DIB EW is essentially a measure of the area of the DIB line profile, it seems more appropriate to us to compare the DIB EW with the bump area rather than the bump height. The bump area differs from the bump height by a factor of $\pi \gamma / 2$. Note that although the central wavelength of the $2175 \AA$ bump is stable, its width $\gamma$ varies substantially (see Whittet 2003): while on average $\gamma \approx 1.0 \mu \mathrm{m}^{-1}, \gamma$ ranges from $\gamma<0.8 \mu \mathrm{m}^{-1}$ (e.g. $\gamma \approx 0.79 \mu \mathrm{m}^{-1}$ for HD 93028) to $\gamma>1.2 \mu \mathrm{m}^{-1}$ (e.g. $\gamma \approx 1.25 \mu \mathrm{m}^{-1}$ for $\xi$ Oph and $\gamma \approx 1.62 \mu \mathrm{m}^{-1}$ for HD 29647).

Does the lack of correlation between the strengths of the $2175 \AA$ bump and DIBs (see $\S 3$ ) imply that their carriers are not related as often suggested in the literature (e.g. see Wu et al. 1981, Witt et al. 1983, Seab \& Snow 1984, Benvenuti \& Porceddu 1989)? or in other words, does this conclusively imply that PAHs are unlikely responsible for both the $2175 \AA$ bump and DIBs?

Cox et al. (2007a) studied the $2175 \AA$ extinction bump and DIBs of the SMC. They reported the detection of DIBs toward one line of sight in the relatively quiescent SMC wing region where the $2175 \AA$ extinction feature is also present. In contrast, neither DIBs nor the $2175 \AA$ bump were detected toward five lines of sight in the SMC bar which pass through active star formation regions. They argued that the carriers of the $2175 \AA$ extinction bump and DIBs may be subject to the same physical and chemical processes: both carriers in the SMC bar could be destroyed by shocks or the hard ambient UV radiation; or alternatively, the harsh environment may prohibit the formation of both carriers. We note that in the Galaxy, some lines of sight also exhibit both weak DIBs and a weak extinction bump (e.g. HD 23532, Witt et al. 1981; HD 29647, Adamson et al. 1991; HD 62542, Snow et al. 2002, Ádámkovics et al. 2005) on a per unit reddening basis, when compared with typical Galactic sightlines.

One may argue that it may not be appropriate to compare the EW of a single DIB with the $2175 \AA$ bump since an individual DIB is more characteristic of a specific PAH molecule, while the $2175 \AA$ bump is unlikely from a single PAH molecule, but rather produced by a cosmic mixture of many individual molecules, radicals, and ions. In view of this, we compare

the sum of the EWs of all nine DIBs $\sum_{j=1}^{N=9} W_{\mathrm{DIB}_{j}}$ with the $2175 \AA$ bump strengths $W_{2175}$ of nine sightlines (for which the data for both the nine DIBs and the bump are available), where $W_{\mathrm{DIB}_{j}}$ is the EW of the $j$-th DIB. As shown in Fig. 3 , the correlation between $\sum_{j=1}^{N=9} W_{\mathrm{DIB}_{j}}$ and $W_{2175}$ is at most very weak. We do not want to over-interpret this as the scatter is rather large and there is only a small number of data points. For a more thorough exploration, we need to include more sightlines (i.e. more data points) and an as complete as possible set of 
DIBs. The latter will allow us to approach the total absorption of DIBs.

PAHs are thought to be ubiquitous and abundant in the ISM, as revealed by the ubiquitous "UIR" emission bands at 3.3, 6.2, 7.7, 8.6 and $11.3 \mu \mathrm{m}$ (Léger \& Puget 1984, Allamandola et al. 1985) which account for over $10 \%$ of the total IR luminosity of the Milky Way and external star-forming galaxies (Draine \& Li 2007, Smith et al. 2007).

PAHs do in general have strong absorption in the $\sim 2000 \AA$ region as a result of the strong $\pi-\pi^{*}$ electronic transition. It is therefore natural to attribute at least part of the $2175 \AA$ extinction feature to PAHs (see Draine 2009). Indeed, a correlation between the $2175 \AA$ hump and the IRAS $12 \mu \mathrm{m}$ emission (dominated by PAHs) was found by Boulanger et al. (1994) in the Chamaeleon cloud, suggesting a common carrier.

While it is true that a single PAH molecule exhibits sharp UV absorption features which are not seen in interstellar space, a cosmic mixture of various individual PAH molecules, radicals, and ions would smooth out the fine structures and produce a broad absorption bump around $\sim 2175 \AA$. This has been demonstrated both experimentally and theoretically. Léger et al. (1989) measured the absorption spectra of mixtures of over 300 neutral PAH species with $\sim 12-28 \mathrm{C}$ atoms obtained from a coal pitch extract evaporated at $\sim 380-480 \mathrm{~K}$. Joblin et al. (1992) measured the absorption spectra of the neutral PAH mixtures containing $\sim 14-44 \mathrm{C}$ atoms obtained from a coal pitch extract evaporated at $\sim 570-630 \mathrm{~K}$. All these spectra have a strong UV feature around $2175 \AA$ (but relatively broader than the interstellar bump). Cecchi-Pestellini et al. (2008) recently showed that a weighted sum of 50 PAHs in the size range of $\sim 10-66 \mathrm{C}$ atoms in the charge states of 0 (neutral), \pm 1 (cation and anion), and +2 (dication) is able to reproduce the $2175 \AA$ extinction bump observed in five sightlines with $R_{V}$ ranging from 2.33 to 5.05 (also see Malloci et al. 2008).

So, why do not DIBs correlate with the extinction bump? A possible explanation might be that DIBs are produced by the smallest gas-phase free-flying PAH molecules and ions, while the $2175 \AA$ bump is mainly from large PAHs or PAH clusters in condensed phase. Therefore, one should not expect the DIBs to be tightly correlated to the $2175 \AA$ bump. Indeed, models that reproduce the $\sim 3-20 \mu \mathrm{m}$ "UIR" emission bands have most of the PAH mass in PAHs with $N_{\mathrm{C}} \gtrsim 10^{2} \mathrm{C}$ atoms (Li \& Draine 2001, Draine \& Li 2007)11 while

\footnotetext{
${ }^{11}$ This scenario naturally explains the lack of correlation between the strengths of the $\lambda 5780 \AA$ and $\lambda 6283 \AA$ DIBs with the Spitzer $8 \mu \mathrm{m}$ IRAC fluxes (which are thought to arise from PAHs) observed in the ISM of M31 (Cordiner et al. 2011): small PAHs (say, with $N_{\mathrm{C}}<30-50 \mathrm{C}$ atoms) which are responsible for the DIBs do not emit much at the $7.7 \mu \mathrm{m} \mathrm{C}-\mathrm{C}$ stretching mode (instead, they probably mainly emit at the $3.3 \mu \mathrm{m}$ C-H stretching mode), only large PAHs with $N_{\mathrm{C}} \gtrsim 10^{2} \mathrm{C}$ atoms dominate the $7.7 \mu \mathrm{m}$ emission (see Fig. 7 of Draine \& Li 2007).
} 
laboratory studies are generally limited to smaller PAHs (e.g. $12<N_{\mathrm{C}}<28$ in Léger et al. 1989, and $14<N_{\mathrm{C}}<44$ in Joblin et al. 1992). This may also be the reason why the PAH samples in the laboratory (Léger et al. 1989, Joblin et al. 1992) do not precisely reproduce the observed profile of the $2175 \AA$ feature: most notably is the feature at $\sim 3000 \AA$ which is seen in the laboratory spectra but not observed in the ISM. Joblin et al. (1992) noted that this feature shifts in the laboratory spectra towards longer wavelengths as molecules become larger. Joblin et al. (1992) argued that one would expect that this feature merges into a continuum when larger species are present in the laboratory mixture. More recently, Steglich et al. (2010) showed that larger PAHs indeed provide better fits to the observed $2175 \AA$ feature. Alternatively, DIBs may mainly arise from small PAH ions while the $2175 \AA$ hump is weakened in cations (e.g. Lee \& Wdowiak 1993). Moreover, PAHs may not be the sole contributor to the $2175 \AA$ bump. Other dust species such as small graphitic dust and carbon buckyonions may also (at least partly) contribute to the bump (see Li et al. 2008). Therefore, although PAHs may indeed be responsible for both the extinction bump and DIBs, their strengths do not have to correlate (i.e. they do not necessarily have a one-to-one correspondence).

Finally, if DIBs are indeed caused by the electronic transitions of PAHs, they hold great promise for identifying specific PAH molecules. So far, not a single individual PAH molecule has been identified in the ISM 12 despite that many unidentified interstellar phenomena (e.g. the $2175 \AA$ bump, DIBs, the so-called blue luminescence [Vijh et al. 2005a,b] and extended red emission [ERE; Witt \& Vijh 2004], the 3.3-11.3 $\mu \mathrm{m}$ emission bands, and the "anomalous" Galactic foreground microwave emission [Draine 2003]) are arguably attributed to PAHs. Neither the $2175 \AA$ extinction bump nor the $3.3-11.3 \mu \mathrm{m}$ "UIR" emission features could allow us to fingerprint individual PAH molecules as they are caused by a mixture of PAH molecules of a range of sizes. Moreover, the mid-IR bands are mostly representative of functional groups, not the molecular frame of a PAH molecule. In contrast, the DIBs and the far-IR vibrational bands are sensitive to the skeletal characteristics of a PAH molecule, hence they contain specific fingerprint information on its identity (Salama et al. 1996, 1999; Joblin et al. 2009, Zhang et al. 2010).

\footnotetext{
${ }^{12}$ The naphthalene cation $\left(\mathrm{C}_{10} \mathrm{H}_{8}^{+}\right)$was tentatively suggested to be responsible for several DIBs (Salama \& Allamandola 1992, Snow 1992, Krełowski et al. 2001, Iglesias-Groth et al. 2008). Iglesias-Groth et al. (2010) also reported a tentative detection of anthracene cation $\left(\mathrm{C}_{14} \mathrm{H}_{10}^{+}\right)$. However, these claimed detections were not confirmed by Galazutdinov et al. (2011).
} 


\section{Summary}

We have explored the relationship between the $2175 \AA$ interstellar extinction bump with nine DIBs, using the area above the linear extinction background as a measure of the bump strength. We have compiled the extinction bump and DIB equivalent width data of a large sample of 84 stars. It is found that the DIB EWs are not correlated with the $2175 \AA$ extinction bump. A possible explanation might be that DIBs are produced by small free gas-phase PAH molecules and ions, while the $2175 \AA$ bump is mainly from large PAHs or PAH clusters in condensed phase.

We thank L.J. Allamandola, S.D. Friedman, J. Gao, J.Y. Hu, S. Kwok, S.L. Liang, B.J. McCall, F. Salama, P.J. Sarre, and A.N. Witt for very helpful discussions and/or comments. We also thank the anonymous referee for his/her very helpful comments and suggestions which substantially improved the quality of this work. FYX is supported in part by the Scientific Research Fund of Hunan Provincial Education Department (No.08A072), NSFC grant No. 10878012, the Xiangtan University Natural Science Foundation (No. 10KZ08044) and the Key Laboratory of Particle Astrophysics, Institute of High Energy Physics, CAS. AL is supported in part by Spitzer Theory Programs and NSF grant AST 07-07866.

\section{A. The Equivalent Widths of Individual DIBs}

In the literature, different equivalent widths have often been reported for a single DIB of a given sightline (see Tables 3-11). In some cases the reported EWs differ substantially from one another 13 It is therefore not trivial to select the "true" EW of a DIB. We employ the following criteria to adopt the EW of a DIB of a given line of sight from the literature: (1) if the sightline has been observed only once, we will adopt the DIB EW reported from that observation; (2) if the sightline has been observed twice, we will take the mean value of the reported EWs if they are close (denoted by " $\sqrt{ }$ " in Tables 3-11); otherwise we will take the one which falls in the $W_{\mathrm{DIB}}-E(B-V)$ linear relation 14 and reject the one which

\footnotetext{
${ }^{13}$ In some cases this is (at least partly) caused by the different wavelength limits of integration used to derive the EW of a DIB. This becomes even more true as the number of DIBs increases. Some authors include certain blends as a single DIB, others do not [e.g., in calculating the EW of the $\lambda 5797 \AA$ DIB, Galazutdinov et al. (2004) did not include the blue wing, while Friedman et al. (2011) did include it].

${ }^{14}$ For a given DIB, we obtain the $W_{\mathrm{DIB}}-E(B-V)$ linear relation by using the $W_{\mathrm{DIB}}$ and $E(B-V)$ data of all 84 sightlines studied here. A linear relation between $W_{\text {DIB }}$ and $E(B-V)$ is expected as both quantities are proportional to the amount of interstellar materials along the line of sight.
} 
deviates significantly from that relation (denoted by " $\times$ " in Tables $3-11$ ); (3) if the sightline has been observed more than twice and for a single DIB there are three or more sets of DIB EWs reported in the literature, we will take the mean value of those reported EWs which are close to each other and reject those which deviate significantly from others.

\section{REFERENCES}

Ádámkovics, M., Blake, G.A., \& McCall, B.J. 2005, ApJ, 625, 857

Adamson, A. J., Whittet, D. C. B., \& Duley, W.W. 1991, MNRAS, 252, 234

Allamandola, L.J., Tielens, A.G.G.M., \& Barker, J.R. 1985, ApJ, 290, L25

Benvenuti, P., \& Porceddu, I. 1989, A\&A, 223, 329

Boulanger, F., Prevot, M. L., \& Gry, C. 1994, A\&A, 284, 956

Cecchi-Pestellini, C., Malloci, G., Mulas, G., Joblin, C., \& Williams, D. A. 2008, A\&A, 486, L25

Conroy, C., Schiminovich, D., \& Blanton, M. R. 2010, ApJ, 718, 184

Cordiner, M. A., Cox, N. L. J., Trundel, R., Evans, C. J., Hunter, I., Przybilla, N., Bresolin, F., \& Salama, F. 2008a, A\&A, 480, 13

Cordiner, M. A., Smith, K. T., Cox, N. L. J., Evans, C. J., Hunter, I., Przybilla, N., Bresolin, F., \& Sarre, P. J. 2008b, A\&A, 492, L5

Cordiner, M. A., Cox, N. L. J., Evans, C. J., Trundle, C., Smith, K. T., Sarre, P. J., \& Gordon, K. D. 2011, ApJ, 726, 39

Cox, N. L. J., Cordiner, M. A., Cami, J., Foing, B. H., Sarre, P. J., Kaper, L., \& Ehrenfreund, P. 2006, A\&A, 447, 991

Cox, N. L. J., et al. 2007a, A\&A, 470, 941

Cox, N. L. J., et al. 2007b, A\&A, 465, 899

Crawford, M. K., Tielens, A. G. G. M., \& Allamandola, L.J. 1985, ApJ, 293, L45

Danks, A. C. 1980, PASP, 92, 52

Désert, F.-X., Jenniskens, P., \& Dennefeld, M. 1995, A\&A, 303, 223 
Dorschner, J., Friedemann, C., \& Guertler, J. 1977, A\&A, 58, 201

Draine, B. T. 1989, in IAU Symp. 135, Interstellar Dust, ed. L. J. Allamandola \& A. G. G. M. Tielens (Dordrecht: Kluwer), 313

Draine, B. T. 2009, EAS Publ. Ser., 35, 245

Draine, B. T. 2003, ARA\&A, 41, 241

Draine, B. T., \& Li, A. 2007, ApJ, 657, 810

Duley, W. W. 2006, ApJ, 639, L59

Ehrenfreund, P., \& Foing, B. H. 1996, A\&A, 307, L25

Ehrenfreund, P., et al. 2002, ApJ, 576, L117

Elíasdóttír, Á., et al. 2009, ApJ, 697, 1725

Ellison, S. L., York, B. A., Murphy, M. T., Zych, B. J., Smith, A. M., \& Sarre, P. J. 2008, MNRAS, 383, L30

Fitzpatrick, E. L., \& Massa, D. 1986, ApJ, 307, 286

Fitzpatrick, E. L., \& Massa, D. 1990, ApJS, 72, 163

Fitzpatrick, E. L., \& Massa, D. 2005, AJ, 130, 1127

Friedman, S. D., et al. 2011, ApJ, 727, 33

Galazutdinov, G. A., Manicó, G., Pirronello, V., \& Krełowski, J. 2004, MNRAS, 355, 169

Galazutdinov, G. A., Lee, B.-C., Song, I.-O., Kazmierczak, M., \& Krełowski, J. 2011, MNRAS, in press

Geminale, A. 2006, PhD thesis, Università degli Studi di Padova

Greenberg, J. M., \& Chlewicki, G. 1983, ApJ, 272, 563

Heckman, T.M., \& Lehnert, M.D. 2000, ApJ, 537, 690

Heger, M. L. 1922, Lick Obs. Bull., 10, 141

Herbig, G. H. 1975, ApJ, 196, 129

Herbig, G. H. 1993, ApJ, 407, 142 
Herbig, G. H. 2000, ApJ, 542, 334

Hobbs, L. M., et al. 2008, ApJ, 680, 1256

Hobbs, L. M., et al. 2009, ApJ, 705, 32

Iglesias-Groth, S., Manchado, A., García-Hernández, D. A., González Hernández, J. I., \& Lambert, D. L. 2008, ApJ, 685, L55

Iglesias-Groth, S., Manchado, A., Rebolo, R., González Hernández, J. I., García-Hernández, D. A., \& Lambert, D. L. 2010, MNRAS, 407, 2157

Inoue, A. K., Buat, V., Burgarella, D., Panuzzo, P., Takeuchi, T. T., \& Iglesias-Páramo, J. 2006, MNRAS, 370, 380

Jenniskens, P., \& Greenberg, J. M. 1993, A\&A, 274, 439

Jiang, P., Ge, J., Zhou, H., Wang, J., \& Wang, T. 2011, ApJ, in press (arXiv:1103.1932)

Joblin, C., Léger, A., \& Martin, P. 1992, ApJ, 393, L79

Joblin, C., Berné, O., Simon, A., \& Mulas, G. 2009, Cosmic Dust - Near and Far (ASP Conf. Ser. 414), ed. T. Henning, E. Grün, \& J. Steinacker (San Francisco, CA: ASP), 383

Junkkarinen, V. T., Cohen, R. D., Beaver, E. A., Burbidge, E. M., Lyons, R. W., \& Madejski, G. 2004, ApJ, 614, 658

Krełowski, J., \& Walker, G. A. H. 1987, ApJ, 312, 860

Krełowski, J., Schmidt, M., \& Snow, T. P. 1997, PASP, 109, 1135

Krełowski, J., Galazutdinov, G. A., Musaev, F. A., \& Nirski, J. 2001, MNRAS, 328, 810

Lawton, B., Churchill, C. W., York, B. A., Ellison, S. L., Snow, T. P., Johnson, R. A., Ryan, S. G., \& Benn, C. R. 2008, AJ, 136, 994

Lee, W., \& Wdowiak, T.J. 1993, ApJ, 410, L127

Léger, A., \& d'Hendecourt, L. 1985, A\&A, 1985, 146, 81

Léger, A., \& Puget, J. 1984, A\&A, 137, L5

Léger, A., Verstraete, L., d'Hendecourt, L., Défourneau, D., Dutuit, O., Schmidt, W., \& Lauer, J. 1989, in Interstellar Dust (IAU Symp. 135), ed. L.J. Allamandola \& A.G.G.M. Tielens (Dordrecht: Kluwer), 173 
Lewis, N. K., Cook, T. A., \& Chakrabarti, S. 2005, ApJ, 619, 357

Li, A., \& Draine, B. T. 2001, ApJ, 554, 778

Li, A., Chen, J. H., Li, M. P., Shi, Q. J., \& Wang, Y. J. 2008, MNRAS, 390, L39

Liang, S. L., \& Li, A. 2009, ApJ, 690, 56

Liang, S. L., \& Li, A. 2010, ApJ, 710, 648

Malloci, G., Mulas, G., Cecchi-Pestellini, C., \& Joblin, C. 2008, A\&A, 489, 1183

McCall, B. J., et al. 2010, ApJ, 708, 1628

Megier, A., Krełowski, J., \& Weselak, T. 2005, MNRAS, 358, 563

Merrill, P. W. 1934, PASP, 46, 206

Motta, V., et al. 2002, ApJ, 574, 719

Nandy, K., \& Thompson, G. I. 1975, MNRAS, 173, 237

Noll, S., \& Pierini, D. 2005, A\&A, 444, 137

Noll, S., Pierini, D., Pannella, M., \& Savaglio, S. 2007, A\&A, 472, 455

Noll, S., et al. 2009, A\&A, 499, 69

Prochaska, J. X., et al. 2009, ApJ, 691, 27

Salama, F., \& Allamandola, L. J. 1992, ApJ, 395, 301

Salama, F., Bakes, E. L. O., Allamandola, L. J., \& Tielens, A. G. G. M. 1996, ApJ, 458, 621

Salama, F., Galazutdinov, G. A., Krełowski, J., Allamandola, L. J., \& Musaev, F. A. 1999, ApJ, 526, 265

Salama, F., Galazutdinov, G. A., Krełowski, J., Biennier, L., Beletsky, Y., \& Song, I.-O. 2011, ApJ, 728, 154

Sarre, P. J. 2006, J. Mol. Spec., 238, 1

Sarre, P. J., Miles, J. R., Kerr, T. H., Hibbins, R. E., Fossey, S. J., \& Somerville, W. B. 1995a, MNRAS, 277, L41

Sarre, P. J., Miles, J. R., \& Scarrott, S. M. 1995b, Science, 269, 674 
Schmidt, E. G. 1978, ApJ, 223, 458

Seab, C. G., \& Snow, T. P. 1984, ApJ, 277, 200

Smith, J. D., et al. 2007, ApJ, 656, 770

Snow, T. P. 1992, ApJ, 401, 775

Snow, T. P., Welty, D. E., Thorburn, J., Hobbs, L. M., McCall, B. J., Sonnentrucker, P., \& York, D. G. 2002, ApJ, 573, 670

Sofia, U. J., et al. 2005, ApJ, 625, 167

Sonnentrucker, P., Cami, J., Ehrenfreund, P., \& Foing, B. H. 1997, A\&A, 327, 1215

Srianand, R., Gupta, N., Petitjean, P., Noterdaeme, P., \& Saikia, D. J. 2008, MNRAS, 391, L69

Stecher, T. P. 1965, ApJ, 142, 1683

Stecher, T. P., \& Donn, B. 1965, ApJ, 142, 1681

Steglich, M., Jäger, C., Rouillé, G., Huisken, F., Mutschke, H., \& Henning, T. 2010, ApJ, 712 , L16

Thorburn, J., et al. 2003, ApJ, 584, 339

Tielens, A. G. G. M. 2008, ARA\&A, 46, 289

Vijh, U.P., Witt, A.N., \& Gordon, K.D. 2005a, ApJ, 619, 368

Vijh, U.P., Witt, A.N., \& Gordon, K.D. 2005b, ApJ, 633, 262

Wang, J., Hall, P. B., Ge, J., Li, A., \& Schneider, D. P. 2004, ApJ, 609, 589

Wegner, W. 2003, Astron. Nachr., 324, 219

Welty, D. E., Federman, S. R., Gredel, R., Thorburn, J. A., \& Lambert, D. L. 2006, ApJS, 165,138

Whittet, D.C.B. 2003, Dust in the Galactic Environment (2nd ed; Bristol: IoP)

Whittet, D. C. B., Shenoy, S. S., Clayton, G. C., \& Gordon, K. D. 2004, ApJ, 602, 291

Witt, A. N., Bohlin, R. C., \& Stecher, T. P. 1981, ApJ, 244, 199 
Witt, A. N., Bohlin, R. C., \& Stecher, T. P. 1983, ApJ, 267, L47

Witt, A. N., \& Vijh, U. P. 2004, in Astrophysics of Dust (ASP Conf. Ser. 309), ed. A. N. Witt, G. C. Clayton, \& B. T. Draine (San Francisco, CA: ASP), 115

Wszołek, B., \& Godłowski, W. 2003, MNRAS, 338, 990

Wu, C. C., York, D. G., \& Snow, T. P. 1981, AJ, 1981, 86, 755

York, B. A., Ellison, S. L., Lawton, B., Churchill, C. W., Snow, T. P., Johnson, R. A., \& Ryan, S. G. 2006a, ApJ, 647, L29

York, D. G., et al. 2006b, MNRAS, 367, 945

van der Zwet, G. P., \& Allamandola, L. J. 1985, A\&A, 146, 76

Zhang, J., Han, F., Pei, L., Kong, W., \& Li, A. 2010, ApJ, 715, 485

Zhou, H., Ge, J., Lu, H., Wang, T., Yuan, W., Jiang, P., \& Shan, H. 2010, ApJ, 708, 742

This preprint was prepared with the AAS LATEX macros v5.2. 


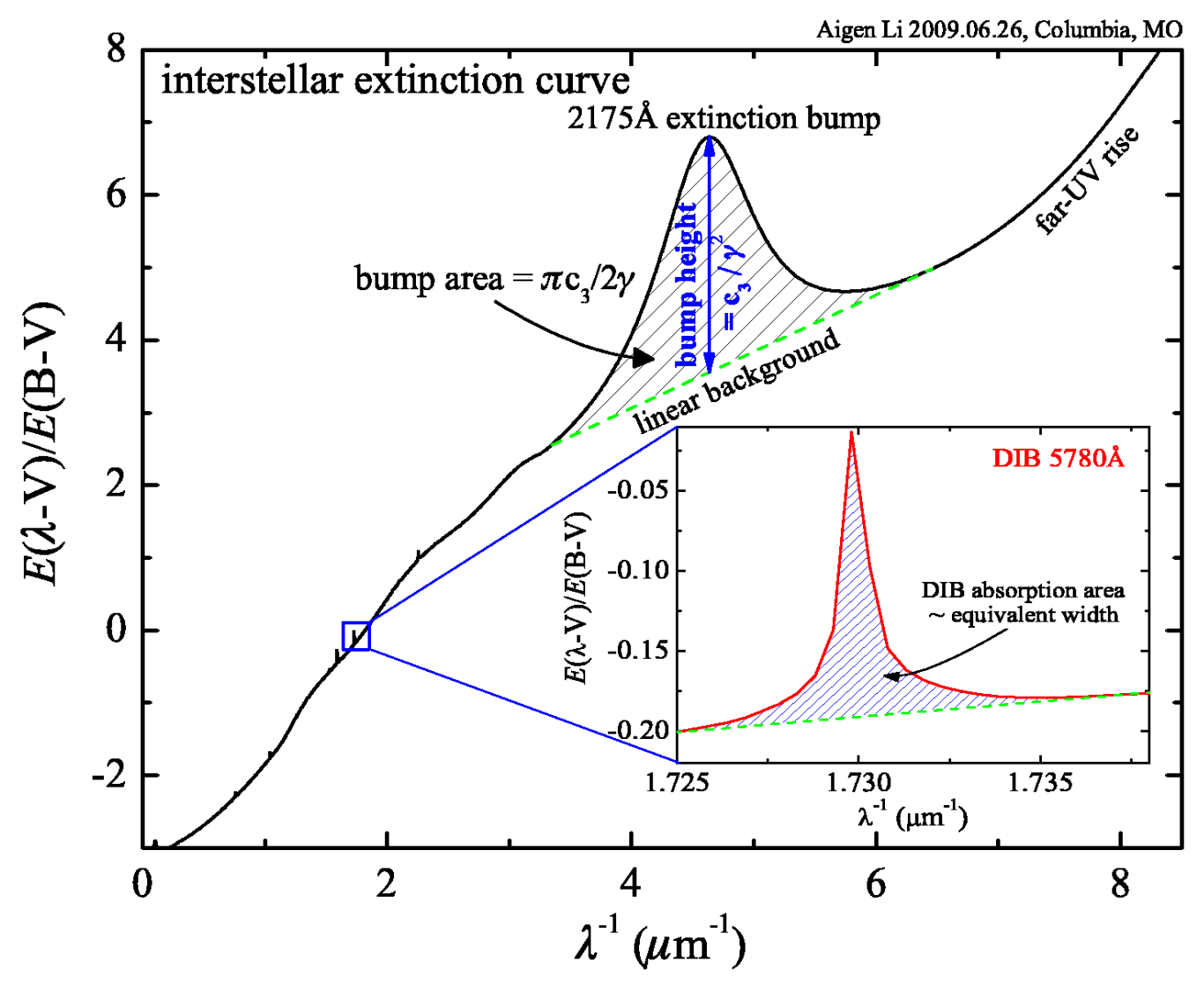

Fig. 1. - Interstellar extinction curve and DIBs. We use the black shaded area $\left(W_{2175} / E(B-V)=\pi c_{3} / 2 \gamma\right)$ above the "linear background extinction" in the FM90 decomposition scheme as a measure of the strength of the $2175 \AA$ extinction bump (normalized by $E(B-V)$ ), while most previous work considered the bump height $\left(h_{2175} / E(B-V)=c_{3} / \gamma^{2}\right)$ as the bump strength. The little sticks in the visible part plot some DIBs. In the inserted panel which illustrates the $\lambda 5780 \AA$ DIB, the blue shaded area represents its absorption strength. 

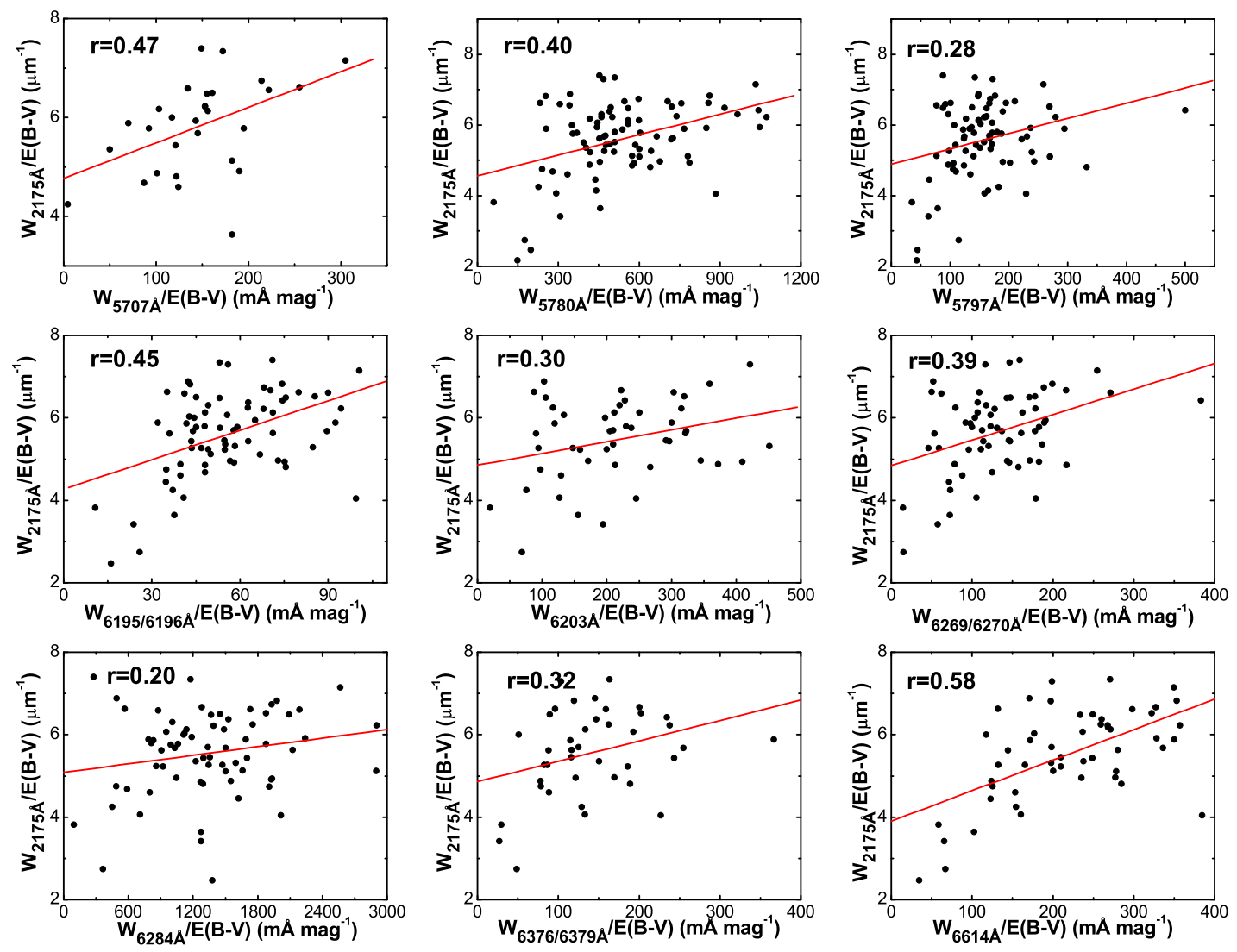

Fig. 2. - Correlation diagrams of the strengths of the $2175 \AA$ extinction bump with the equivalent widths of the DIBs at $\lambda 5707 \AA, \lambda 5780 \AA, \lambda 5797 \AA, \lambda 6195 \AA / 6196 \AA, \lambda 6203 \AA, \lambda 6269 \AA / 6270 \AA, \lambda 6284 \AA$, $\lambda 6376 \AA / 6379 \AA$, and $\lambda 6614 \AA$. The $\lambda 6195 \AA / 6196 \AA$ DIB is a single DIB, although in the literature it is listed either as $\lambda 6195 \AA$ or $\lambda 6196 \AA$. This is also true for the $\lambda 6269 \AA / 6270 \AA$ DIB. The two DIBs at $\lambda 6376 \AA$ and $\lambda 6379 \AA$ are grouped into one DIB (" $\lambda 6376 \AA / 6379 \AA$ ") as they may blend. 


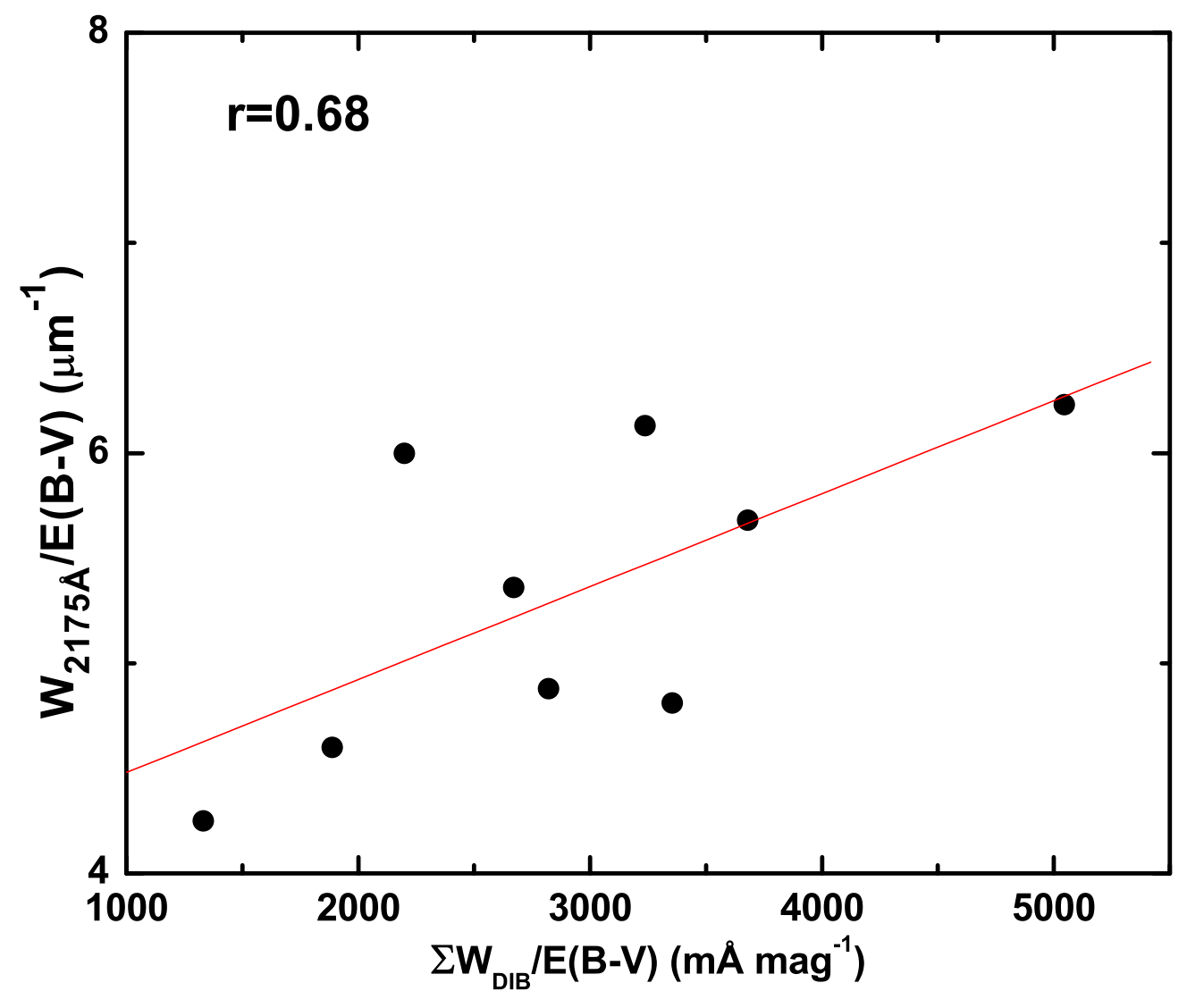

Fig. 3.- Correlation diagram of the strengths of the $2175 \AA$ extinction bump with the sum of the equivalent widths of all nine DIBs $\left(\sum_{j=1}^{N=9} W_{\mathrm{DIB}_{j}}\right)$ for nine interstellar sightlines. All quantities are normalized to $E(B-V)$. 
Table 1. The $2175 \AA$ extinction bump strengths $\left(W_{2175}\right)$ and DIB equivalent widths $\left(W_{\text {DIB }}\right)$ for 84 interstellar sightlines. Sources for $E(B-V)$ and $W_{\text {DIB }}$ are Dorschner et al. (1977), Herbig (1975, 1993, 2000), Seab \& Snow (1984), Benvenuti \& Porceddu (1989), Désert et al. (1995), Sonnentrucker al. (1997), Snow et al. (2002), Wszolk \& Godlowski (2003), Thorburn et al. (2003), Galazutdinov et al. (2004), Megier et al. (2005), Cox et al. (2007b), Hobbs et al. (2008, 2009), McCall et al. (2010), and Friedman et al. (2011). See Appendix and Tables 3-11 for details about how we obtain the DIB equivalent widths adopted here. Sources for $R_{V}$ : ${ }^{(1)}$ Wegner (2003), and ${ }^{(2)}$ Geminale (2006); $c_{3}$ and $\gamma$ are ${ }^{(3)}$ Jenniskens \& Greenberg (1993), (4)

Fitzpatrick \& Massa (2005), ${ }^{(5)}$ Whittet et al. (2004), ${ }^{(6)}$ Fitzpatrick \& Massa (1990), ${ }^{(7)}$ Lewis et al. (2005), and ${ }^{(8)}$ Sofia et al. (2005).

\begin{tabular}{|c|c|c|c|c|c|c|c|c|c|c|c|c|c|c|}
\hline $\mathrm{HD} / \mathrm{BD}$ & $\begin{array}{c}E(B-V) \\
(\mathrm{mag})\end{array}$ & $R_{V}$ & $\begin{array}{c}c_{3} \\
\left(\mu \mathrm{m}^{-2}\right)\end{array}$ & $\begin{array}{c}\gamma \\
\left(\mu \mathrm{m}^{-1}\right) \\
\end{array}$ & $\begin{array}{c}W_{2175} / E(B-V) \\
\left(\mu \mathrm{m}^{-1}\right)\end{array}$ & $\begin{array}{c}W_{5707} \\
(\mathrm{~m} \AA)\end{array}$ & $\begin{array}{c}W_{5780} \\
(\mathrm{~m} \AA)\end{array}$ & $\begin{array}{c}W_{5797} \\
(\mathrm{~m} \AA)\end{array}$ & $\begin{array}{c}W_{6195 / 6196} \\
(\mathrm{~m} \AA)\end{array}$ & $\begin{array}{c}W_{6203} \\
(\mathrm{~m} \AA)\end{array}$ & $\begin{array}{c}W_{6269 / 6270} \\
(\mathrm{~m} \AA)\end{array}$ & $\begin{array}{c}W_{6284} \\
(\mathrm{~m} \AA)\end{array}$ & $\begin{array}{c}W_{6376 / 6379} \\
(\mathrm{~m} \AA)\end{array}$ & $\begin{array}{c}W_{6614} \\
(\mathrm{~m} \AA)\end{array}$ \\
\hline 2905 & 0.33 & $3.26 \pm 0.20^{1}$ & $2.04^{3}$ & $0.79^{3}$ & 4.05 & - & 306.3 & 75.7 & 32.8 & 81.0 & 59.0 & 665.0 & 74.8 & 126.9 \\
\hline 15558 & 0.75 & $3.09 \pm 0.06^{1}$ & $3.46^{3}$ & $0.94^{3}$ & 5.78 & 146.3 & 452.3 & 129.0 & 44.3 & - & 137.3 & 1407.0 & - & - \\
\hline 15570 & 1.02 & $3.30 \pm 0.05^{1}$ & $3.34^{3}$ & $0.95^{3}$ & 5.52 & - & 519.2 & 161.2 & - & - & - & - & - & - \\
\hline 16691 & 0.83 & - & $3.97^{3}$ & $0.95^{3}$ & 6.56 & 184.3 & 283.9 & 63.9 & - & - & - & - & - & - \\
\hline 21291 & 0.43 & $3.43 \pm 0.10^{1}$ & $4.98^{3}$ & $1.07^{3}$ & 7.31 & - & 196.5 & 72.5 & 23.5 & 177.0 & 48.8 & _- & 43.5 & 83.5 \\
\hline 21483 & 0.56 & $3.05 \pm 0.12^{1}$ & $2.85^{4}$ & $1.10^{4}$ & 4.07 & - & 164.0 & 89.0 & 22.9 & 71.0 & 59.0 & 397.0 & 74.5 & 89.9 \\
\hline 23060 & 0.32 & $3.02 \pm 0.19^{1}$ & $4.17^{3}$ & $1.06^{3}$ & 6.18 & 35.0 & 137.5 & 42.0 & - & - & - & - & - & 88.5 \\
\hline 27778 & 0.37 & - & $3.57^{4}$ & $1.18^{4}$ & 4.75 & - & 84.0 & 36.7 & 12.2 & 34.0 & 10.0 & 170.0 & 27.5 & 43.9 \\
\hline 29647 & 1.00 & - & $3.84^{5}$ & $1.58^{5}$ & 3.82 & - & 60.8 & 36.0 & 10.9 & 20.0 & 14.6 & 95.0 & 29.9 & 59.7 \\
\hline 30614 & 0.26 & $2.83 \pm 0.16^{1}$ & $3.21^{3}$ & $0.94^{3}$ & 5.36 & 16.0 & 121.0 & 45.3 & 16.5 & 63.0 & 56.0 & 367.1 & 45.1 & 71.3 \\
\hline+31643 & 0.85 & - & $5.37^{3}$ & $1.28^{3}$ & 6.59 & 113.9 & 259.3 & 78.2 & 34.9 & - & 52.7 & 746.3 & - & - \\
\hline 34078 & 0.49 & $3.27 \pm 0.14^{1}$ & $4.15^{6}$ & $1.09^{6}$ & 6.00 & 60.8 & 182.0 & 55.6 & 23.1 & 102.5 & 53.8 & 578.5 & 26.4 & 61.0 \\
\hline 36879 & 0.50 & - & $2.38^{3}$ & $0.76^{3}$ & 4.92 & 95.0 & 291.0 & 53.0 & 29.0 & - & 73.0 & 962.0 & - & - \\
\hline 37022 & 0.34 & $5.37 \pm 0.22^{1}$ & $1.33^{6}$ & $0.85^{6}$ & 2.47 & - & 67.3 & 15.1 & 5.5 & - & - & 468.0 & - & 11.7 \\
\hline 37023 & 0.37 & - & $1.22^{6}$ & $0.88^{6}$ & 2.18 & - & 55.0 & 16.0 & - & - & - & - & - & $\mathrm{s}_{-}^{+}$ \\
\hline 37061 & 0.56 & $4.14 \pm 0.14^{1}$ & $2.29^{3}$ & $1.05^{3}$ & 3.42 & - & 162.4 & 33.3 & 12.6 & 103.0 & 30.5 & 675.0 & 14.3 & 34.7 \\
\hline 37367 & 0.40 & $2.74 \pm 0.75^{2}$ & $4.28^{3}$ & $0.94^{3}$ & 7.15 & 131.2 & 433.4 & 108.7 & 42.2 & - & 107.1 & 1078.0 & - & 146.9 \\
\hline 37903 & 0.35 & $3.74 \pm 0.16^{1}$ & $2.25^{4}$ & $0.97^{4}$ & 3.64 & 69.2 & 164.0 & 28.5 & 13.5 & 56.0 & 26.0 & 458.4 & _- & 36.9 \\
\hline 38087 & 0.29 & $5.21 \pm 0.26^{1}$ & $3.65^{3}$ & $0.95^{3}$ & 6.03 & - & 162.0 & 44.0 & 12.4 & - & - & 325.0 & - & 51.3 \\
\hline 38131 & 0.51 & $3.05 \pm 0.14^{1}$ & $4.00^{3}$ & $0.95^{3}$ & 6.61 & 130.1 & 386.1 & 85.2 & 45.9 & - & 138.2 & 1115.9 & - & - \\
\hline 40893 & 0.46 & $3.37 \pm 0.64^{2}$ & $3.13^{3}$ & $0.83^{3}$ & 5.92 & - & 391.0 & 109.0 & 39.0 & - & - & 1030.0 & - & 151.0 \\
\hline 41117 & 0.44 & $3.28 \pm 0.14^{1}$ & $3.64^{3}$ & $0.97^{3}$ & 5.89 & - & 345.5 & 132.6 & 41.6 & 135.0 & 85.3 & 760.0 & 165.0 & 157.6 \\
\hline 42087 & 0.35 & $3.16 \pm 0.16^{1}$ & $4.36^{3}$ & $1.05^{3}$ & 6.52 & - & 259.4 & 96.9 & 30.8 & 115.0 & 64.0 & 675.0 & 72.8 & 116.0 \\
\hline 46056 & 0.50 & $3.06 \pm 0.11^{1}$ & $3.03^{6}$ & $0.93^{6}$ & 5.11 & - & 300.0 & 135.0 & 33.4 & - & - & 750.0 & - & 139.0 \\
\hline 46106 & 0.42 & - & $3.48^{3}$ & $0.92^{3}$ & 5.94 & - & 440.0 & - & - & - & - & - & - & - \\
\hline 46150 & 0.46 & $3.24 \pm 0.14^{1}$ & $3.32^{3}$ & $0.93^{3}$ & 5.60 & - & 331.0 & 102.0 & - & - & - & - & - & - \\
\hline 46202 & 0.49 & $3.23 \pm 0.14^{1}$ & $2.54^{6}$ & $0.84^{6}$ & 4.74 & - & 332.0 & 119.0 & 35.8 & 169.0 & 84.0 & 935.0 & 83.0 & 135.9 \\
\hline 47129 & 0.34 & - & $4.27^{3}$ & $1.05^{3}$ & 6.38 & - & 166.8 & 64.4 & 21.3 & - & 36.4 & 550.0 & 49.9 & 88.4 \\
\hline 48099 & 0.28 & $3.55 \pm 0.17^{1}$ & $2.98^{4}$ & $0.83^{4}$ & 5.63 & - & 203.0 & 47.0 & 19.9 & 90.0 & 45.0 & 595.0 & 32.6 & 78.4 \\
\hline 48434 & 0.28 & $2.60 \pm 0.56^{2}$ & $4.22^{6}$ & $1.05^{6}$ & 6.31 & - & 270.0 & - & - & - & - & - & - & - \\
\hline+60497 & 0.89 & $3.16 \pm 0.08^{1}$ & $3.71^{3}$ & $0.98^{3}$ & 5.94 & 127.3 & 395.2 & 121.9 & 57.9 & - & 170.0 & 1057.3 & - & - \\
\hline+60594 & 0.62 & $2.78 \pm 0.45^{2}$ & $3.77^{3}$ & $0.95^{3}$ & 6.23 & - & 285.0 & 98.0 & - & - & - & - & - & 166.0 \\
\hline 122879 & 0.34 & $3.29 \pm 0.14^{1}$ & $4.35^{3}$ & $1.00^{3}$ & 6.83 & - & 292.7 & 60.1 & 25.3 & 122.0 & 67.8 & 672.0 & 40.6 & 120.0 \\
\hline 123008 & 0.63 & $\begin{array}{c}0.20 .14 \\
-\end{array}$ & $2.77^{3}$ & $0.88^{3}$ & 4.94 & - & 495.8 & 127.3 & 47.3 & 258.0 & 115.3 & 1217.8 & - & - \\
\hline 142096 & 0.19 & $4.17 \pm 1.37^{2}$ & $2.47^{4}$ & $0.87^{4}$ & 4.46 & - & 83.1 & 12.3 & 6.6 & - & 13.6 & 308.0 & - & 23.3 \\
\hline 143018 & 0.05 & - & $3.23^{7}$ & $0.99^{7}$ & 5.12 & - & 39.0 & 7.0 & 2.5 & - & - & 145.0 & - & 10.0 \\
\hline 143275 & 0.17 & $3.09 \pm 0.27^{1}$ & $2.59^{7}$ & $0.77^{7}$ & 5.27 & _- & 80.0 & 21.5 & 7.4 & 16.0 & 10.0 & 250.0 & 14.0 & 22.5 \\
\hline 144217 & 0.17 & $2.72 \pm 0.23^{1}$ & $2.73^{7}$ & $0.66^{7}$ & 6.49 & - & 164.9 & 15.8 & 13.6 & 20.0 & 28.0 & 397.0 & 17.0 & 44.9 \\
\hline 144470 & 0.19 & $3.35 \pm 0.20^{1}$ & $3.43^{7}$ & $0.81^{7}$ & 6.62 & - & 180.0 & 41.0 & 16.8 & 63.7 & 22.9 & 363.0 & 33.4 & 62.6 \\
\hline 145502 & 0.25 & $3.48 \pm 0.14^{1}$ & $3.58^{7}$ & $0.90^{7}$ & 6.25 & - & 184.5 & 40.5 & 15.7 & 28.0 & 19.0 & 421.0 & 39.0 & 64.8 \\
\hline 147165 & 0.34 & $3.22 \pm 0.15^{1}$ & $2.77^{7}$ & $0.83^{7}$ & 5.27 & - & 244.8 & 37.4 & 17.8 & 56.0 & 17.5 & 498.0 & 32.8 & 62.9 \\
\hline 147888 & 0.47 & $3.77 \pm 0.13^{1}$ & $3.82^{6}$ & $1.02^{6}$ & 5.87 & - & 253.0 & 57.5 & 19.6 & 56.0 & 46.0 & 390.0 & 54.0 & 80.8 \\
\hline 147889 & 1.08 & $3.68 \pm 0.03^{1}$ & $4.91^{3}$ & $1.12^{3}$ & 6.88 & _- & 370.5 & 160.0 & 45.8 & 111.5 & 56.0 & 530.0 & 157.0 & 184.5 \\
\hline 147933 & 0.46 & $4.40 \pm 0.12^{1}$ & $3.65^{3}$ & $1.02^{3}$ & 5.62 & - & 212.3 & 58.2 & 16.9 & 42.5 & 25.0 & 426.0 & 41.4 & 67.8 \\
\hline 149757 & 0.29 & $2.92 \pm 0.22^{1}$ & $5.84^{6}$ & $1.38^{6}$ & 6.63 & - & 71.8 & 31.2 & 10.9 & 27.0 & 15.6 & 175.0 & 29.8 & 40.8 \\
\hline 152233 & 0.45 & $3.42 \pm 0.14^{1}$ & $3.67^{3}$ & $1.00^{3}$ & 5.76 & - & 158.9 & 84.2 & 23.9 & 107.0 & 59.0 & 446.9 & - & - \\
\hline 152247 & $\begin{array}{l}0.45 \\
0.44\end{array}$ & $3.15 \pm 0.13^{1}$ & $\begin{array}{l}3.01 \\
3.51^{3}\end{array}$ & $0.95^{3}$ & 5.80 & - & 224.0 & 79.2 & 21.1 & 101.0 & 54.1 & 356.8 & - & - \\
\hline 152248 & 0.45 & - & $3.10^{3}$ & $0.93^{3}$ & 5.24 & - & 228.1 & - & 22.1 & 90.0 & 50.0 & 385.2 & - & - \\
\hline 152249 & 0.46 & $3.22 \pm 0.17^{1}$ & $3.98^{3}$ & $1.02^{3}$ & 6.13 & _- & 257.1 & 68.1 & 22.1 & 115.0 & 49.2 & 523.9 & - & - \\
\hline 154445 & 0.39 & $3.15 \pm 0.19^{1}$ & $4.91^{3}$ & $1.05^{3}$ & 7.34 & 74.0 & 198.9 & 55.5 & 20.7 & - & 57.2 & 458.8 & 63.6 & 105.6 \\
\hline
\end{tabular}


Table 1-Continued

\begin{tabular}{|c|c|c|c|c|c|c|c|c|c|c|c|c|c|c|}
\hline $\mathrm{HD} / \mathrm{BD}$ & $\begin{array}{c}E(B-V) \\
\quad(\mathrm{mag})\end{array}$ & $R_{V}$ & $\begin{array}{c}c_{3} \\
\left(\mu \mathrm{m}^{-2}\right)\end{array}$ & $\begin{array}{c}\gamma \\
\left(\mu \mathrm{m}^{-1}\right)\end{array}$ & $\begin{array}{c}W_{2175} / E(B-V) \\
\left(\mu \mathrm{m}^{-1}\right)\end{array}$ & $\begin{array}{c}W_{5707} \\
(\mathrm{~m} \AA)\end{array}$ & $\begin{array}{c}W_{5780} \\
(\mathrm{~m} \AA)\end{array}$ & $\begin{array}{c}W_{5797} \\
(\mathrm{~m} \AA)\end{array}$ & $\begin{array}{c}W_{6195 / 6196} \\
(\mathrm{~m} \AA)\end{array}$ & $\begin{array}{c}W_{6203} \\
(\mathrm{~m} \AA)\end{array}$ & $\begin{array}{c}W_{6269 / 6270} \\
(\mathrm{~m} \AA)\end{array}$ & $\begin{array}{c}W_{6284} \\
(\mathrm{~m} \AA)\end{array}$ & $\begin{array}{c}W_{6376 / 6379} \\
(\mathrm{~m} \AA)\end{array}$ & $\begin{array}{c}W_{6614} \\
(\mathrm{~m} \AA)\end{array}$ \\
\hline 162978 & 0.38 & $3.64 \pm 0.16^{1}$ & $3.22^{3}$ & $0.95^{3}$ & 5.32 & - & 210.0 & 59.0 & 20.4 & 158.0 & 42.1 & 558.0 & - & 69.2 \\
\hline 164492 & 0.32 & $5.79 \pm 0.46^{2}$ & $4.59^{3}$ & $1.08^{3}$ & 6.67 & - & 226.0 & - & - & - & - & - & - & - \\
\hline 164794 & 0.36 & $3.57 \pm 0.17^{1}$ & $3.98^{3}$ & $0.99^{3}$ & 6.31 & - & 161.0 & 34.0 & 17.2 & 77.0 & 41.0 & 352.1 & - & - \\
\hline 165052 & 0.46 & $3.43 \pm 0.16^{1}$ & $3.47^{3}$ & $0.96^{3}$ & 5.68 & - & 211.2 & 55.9 & 19.8 & 92.0 & 80.1 & 464.2 & - & - \\
\hline 166937 & 0.26 & - & $3.77^{3}$ & $0.95^{3}$ & 6.23 & 78.0 & 279.0 & 72.7 & 24.5 & 82.0 & 46.3 & 754.7 & 61.8 & 92.8 \\
\hline 167971 & 1.08 & $3.24 \pm 0.05^{1}$ & $2.81^{8}$ & $0.81^{8}$ & 5.46 & - & 525.6 & 183.0 & 58.5 & 312.0 & 155.6 & 1450.0 & 124.0 & 224.2 \\
\hline 168076 & 0.78 & $3.53 \pm 0.06^{1}$ & $2.85^{3}$ & $0.93^{3}$ & 4.81 & 97.6 & 513.1 & 265.6 & 60.5 & 213.5 & 120.5 & 1033.4 & 151.0 & 227.7 \\
\hline 168112 & 1.01 & $3.17 \pm 0.04^{1}$ & $2.98^{3}$ & $0.86^{3}$ & 5.44 & 125.8 & 490.4 & 112.3 & 44.7 & - & 117.5 & 1335.4 & - & - \\
\hline 183143 & 1.28 & $3.27 \pm 0.02^{1}$ & $3.63^{3}$ & $0.93^{3}$ & 6.13 & 193.4 & 760.5 & 189.3 & 90.3 & 268.7 & 206.3 & 1888.0 & 169.4 & 344.1 \\
\hline 185418 & 0.50 & $3.33 \pm 0.59^{2}$ & $3.94^{6}$ & $0.93^{6}$ & 6.67 & - & 273.0 & 105.0 & 35.2 & 111.0 & 108.0 & 640.0 & 100.0 & 163.5 \\
\hline 190603 & 0.71 & $3.12 \pm 0.06^{1}$ & $2.86^{3}$ & $0.92^{3}$ & 4.88 & 94.9 & 341.5 & 79.5 & 32.5 & 264.0 & 64.2 & 1270.9 & 64.0 & 101.7 \\
\hline 192281 & 0.73 & - & $3.50^{3}$ & $0.95^{3}$ & 5.78 & 67.2 & 268.6 & 102.9 & 32.9 & - & 73.0 & 773.1 & - & - \\
\hline 193322 & 0.41 & $2.85 \pm 0.15^{1}$ & $2.26^{6}$ & $0.86^{6}$ & 4.15 & - & 180.5 & 67.5 & - & - & - & - & - & - \\
\hline 193682 & 0.83 & - & $4.95^{3}$ & $1.05^{3}$ & 7.40 & 123.7 & 376.0 & 73.0 & 58.9 & - & 132.0 & 229.1 & - & $1-$ \\
\hline 197770 & 0.58 & $3.15 \pm 0.56^{2}$ & $5.36^{3}$ & $1.23^{3}$ & 6.82 & - & 146.7 & 85.8 & 24.9 & - & - & - & - & $\mathrm{NO} 4.3$ \\
\hline 198478 & 0.54 & $3.14 \pm 0.09^{1}$ & $3.12^{3}$ & $0.90^{3}$ & 5.44 & - & 316.2 & 77.8 & 33.9 & 160.0 & 79.2 & 919.0 & 131.3 & $\Gamma_{34.3}$ \\
\hline 199216 & 0.73 & $2.76 \pm 0.07^{1}$ & $3.52^{7}$ & $0.94^{7}$ & 5.89 & 51.1 & 186.2 & 97.1 & 23.4 & - & 67.2 & 573.8 & - & $1-$ \\
\hline 199478 & 0.47 & $2.97 \pm 0.09^{1}$ & $4.13^{3}$ & $1.01^{3}$ & 6.42 & - & 490.0 & 235.0 & 35.0 & 107.0 & 180.0 & - & 110.0 & - \\
\hline 199579 & 0.38 & $2.87 \pm 0.17^{1}$ & $2.92^{6}$ & $1.00^{6}$ & 4.60 & 47.1 & 123.8 & 49.7 & 14.7 & 46.5 & 32.5 & 295.1 & 32.7 & 56.7 \\
\hline 203938 & 0.66 & $3.18 \pm 0.07^{1}$ & $3.67^{7}$ & $1.01^{7}$ & 5.70 & - & 332.0 & 117.0 & 40.5 & 151.0 & 78.6 & 936.0 & 87.5 & 138.8 \\
\hline 204827 & 1.06 & $2.89 \pm 0.11^{1}$ & $2.68^{4}$ & $0.99^{4}$ & 4.25 & 5.0 & 247.0 & 199.0 & 40.4 & 83.9 & 79.5 & 488.9 & 140.3 & 168.0 \\
\hline 206165 & 0.48 & $3.07 \pm 0.14^{1}$ & $4.06^{3}$ & $1.05^{3}$ & 6.07 & - & 209.0 & 80.7 & 26.2 & 62.9 & 57.7 & 486.0 & 90.7 & 111.3 \\
\hline 206267 & 0.49 & $3.08 \pm 0.10^{1}$ & $2.88^{7}$ & $0.91^{7}$ & 4.96 & - & 231.5 & 96.7 & 28.9 & 89.0 & 73.0 & 544.0 & 61.9 & 120.0 \\
\hline 207198 & 0.56 & $2.82 \pm 0.06^{1}$ & $3.13^{3}$ & $0.94^{3}$ & 5.23 & - & 246.2 & 141.0 & 32.3 & 93.5 & 56.7 & 543.0 & 109.6 & 123.8 \\
\hline 209339 & 0.37 & $3.15 \pm 0.83^{2}$ & $4.38^{3}$ & $1.02^{3}$ & 6.74 & 79.2 & 220.9 & 62.2 & 25.2 & - & 69.9 & 713.0 & - & - \\
\hline 210121 & 0.40 & - & $1.91^{8}$ & $1.09^{8}$ & 2.74 & - & 70.0 & 46.0 & 10.3 & 27.5 & 6.0 & 146.0 & 19.4 & 26.7 \\
\hline 216532 & 0.87 & $3.21 \pm 0.34^{2}$ & $4.47^{3}$ & $1.08^{3}$ & 6.50 & 140.1 & 430.7 & 119.2 & 39.2 & - & 148.8 & 1259.8 & - & - \\
\hline 216898 & 0.88 & $2.94 \pm 0.06^{1}$ & $3.88^{3}$ & $0.98^{3}$ & 6.22 & 134.6 & 440.0 & 140.8 & 59.8 & - & 112.6 & 1223.2 & - & - \\
\hline 217086 & 0.92 & $3.21 \pm 0.04^{1}$ & $4.17^{3}$ & $1.01^{3}$ & 6.48 & 142.6 & 513.5 & 149.2 & 48.8 & - & 131.6 & 1258.6 & - & 215.0 \\
\hline 229196 & 1.22 & - & $3.79^{3}$ & $1.08^{3}$ & 5.51 & - & 481.0 & - & - & - & - & - & - & - \\
\hline 239729 & 0.67 & $3.18 \pm 0.08^{1}$ & $3.22^{7}$ & $1.08^{7}$ & 4.68 & 58.3 & 186.9 & 73.7 & 32.2 & - & 83.8 & 393.3 & - & - \\
\hline 242908 & 0.62 & - & $2.94^{3}$ & $0.90^{3}$ & 5.13 & 112.8 & 355.9 & 47.7 & - & - & - & 1029.8 & - & - \\
\hline 303308 & 0.46 & - & $2.91^{3}$ & $0.94^{3}$ & 4.86 & - & 264.0 & 57.0 & 22.1 & 98.0 & 99.8 & 584.7 & - & - \\
\hline+631964 & 0.96 & $3.15 \pm 0.32^{2}$ & $3.29^{3}$ & $0.91^{3}$ & 5.68 & 137.8 & 645.1 & 223.8 & 86.8 & 313.0 & 132.9 & 1457.6 & 246.9 & 325.9 \\
\hline
\end{tabular}


Table 2. Correlation coefficients of the $2175 \AA$ extinction bump with nine DIBs.

\begin{tabular}{ccccccccccc}
\hline \hline & $\frac{W_{5707}}{E(B-V)}$ & $\frac{W_{5780}}{E(B-V)}$ & $\frac{W_{5797}}{E(B-V)}$ & $\frac{W_{6195 / 6196}}{E(B-V)}$ & $\frac{W_{6203}}{E(B-V)}$ & $\frac{W_{6269 / 6270}}{E(B-V)}$ & $\frac{W_{6284}}{E(B-V)}$ & $\frac{W_{6376 / 6379}}{E(B-V)}$ & $\frac{W_{6614}}{E(B-V)}$ & $\frac{\sum_{j=1}^{N=9} W\left(\mathrm{DIB}_{j}\right)}{E(B-V)}$ \\
\hline$\frac{W_{2175}}{E(B-V)}$ & +0.47 & +0.40 & +0.28 & +0.45 & +0.30 & +0.39 & +0.20 & +0.32 & +0.58 & +0.68 \\
\hline
\end{tabular}

Table 3. Sources for the equivalent widths of the $\lambda 5707 \AA$ DIB. Those adopted for deriving the "mean" equivalent widths used in this work are denoted by " $\sqrt{ }$ " (otherwise by " $\times$ "). See Appendix for details.

\begin{tabular}{|c|c|c|c|c|c|c|}
\hline $\mathrm{HD} / \mathrm{BD}$ & $\begin{array}{c}E(B-V) \\
\quad(\mathrm{mag})\end{array}$ & $\begin{array}{c}W_{2175} / E(B-V) \\
\left(\mu \mathrm{m}^{-1}\right)\end{array}$ & $\begin{array}{c}W_{5707} \\
(\mathrm{~m} \AA)\end{array}$ & $\begin{array}{c}\text { Adopt " } \sqrt{ } " \\
\text { or Reject "X" }\end{array}$ & Sources & $\begin{array}{c}W_{5707}(\mathrm{~m} \AA) \\
\text { This Work }\end{array}$ \\
\hline 15558 & 0.75 & 5.78 & $146.3 \pm 8.3$ & $\sqrt{ }$ & Désert et al. (1995) & 146.3 \\
\hline 16691 & 0.83 & 6.56 & $184.3 \pm 16.6$ & $\sqrt{ }$ & Désert et al. (1995) & 184.3 \\
\hline 23060 & 0.34 & 6.18 & $35.0 \pm 1.4$ & $\sqrt{ }$ & Désert et al. (1995) & 35.0 \\
\hline 30614 & 0.32 & 5.36 & $16.0 \pm 2.6$ & $\sqrt{ }$ & Désert et al. (1995) & 16.0 \\
\hline+31643 & 0.85 & 6.59 & $113.9 \pm 5.1$ & $\sqrt{ }$ & Désert et al. (1995) & 113.9 \\
\hline 34078 & 0.52 & 6.00 & $60.8 \pm 7.3$ & $\sqrt{ }$ & Désert et al. (1995) & 60.8 \\
\hline 36879 & 0.50 & 4.92 & $95.0 \pm 11.5$ & $\sqrt{ }$ & Désert et al. (1995) & 95.0 \\
\hline 37367 & 0.43 & 7.15 & $131.2 \pm 18.5$ & $\sqrt{ }$ & Désert et al. (1995) & 131.2 \\
\hline 37903 & 0.38 & 3.64 & $69.2 \pm 11.0$ & $\sqrt{ }$ & Désert et al. (1995) & 69.2 \\
\hline 38131 & 0.51 & 6.61 & $130.1 \pm 15.3$ & $\sqrt{ }$ & Désert et al. (1995) & 130.1 \\
\hline+60497 & 0.89 & 5.94 & $127.3 \pm 9.8$ & $\sqrt{ }$ & Désert et al. (1995) & 127.3 \\
\hline 154445 & 0.43 & 7.34 & $74.0 \pm 12.0$ & $\sqrt{ }$ & Désert et al. (1995) & 74.0 \\
\hline 166937 & 0.51 & 6.23 & $78.0 \pm 7.7$ & $\sqrt{ }$ & Désert et al. (1995) & 78.0 \\
\hline 168076 & 0.80 & 4.81 & $97.6 \pm 9.6$ & $\sqrt{ }$ & Désert et al. (1995) & 97.6 \\
\hline 168112 & 1.04 & 5.44 & $125.8 \pm 14.6$ & $\sqrt{ }$ & Désert et al. (1995) & 125.8 \\
\hline 183143 & 1.24 & 6.13 & $193.4 \pm 14.9$ & $\sqrt{ }$ & Désert et al. (1995) & 193.4 \\
\hline 183143 & 1.27 & 6.13 & $2.4 \pm 0.5$ & $\times$ & Hobbs et al. (2009) & \\
\hline 190603 & 0.94 & 4.88 & $94.9 \pm 3.8$ & $\sqrt{ }$ & Désert et al. (1995) & 94.9 \\
\hline 192281 & 0.73 & 5.78 & $67.2 \pm 6.6$ & $\sqrt{ }$ & Désert et al. (1995) & 67.2 \\
\hline 193682 & 0.83 & 7.40 & $123.7 \pm 10.0$ & $\sqrt{ }$ & Désert et al. (1995) & 123.7 \\
\hline 199216 & 0.73 & 5.89 & $51.1 \pm 3.7$ & $\sqrt{ }$ & Désert et al. (1995) & 51.1 \\
\hline 199579 & 0.38 & 4.60 & $47.1 \pm 6.5$ & $\sqrt{ }$ & Désert et al. (1995) & 47.1 \\
\hline 204827 & 1.11 & 4.25 & $5.0 \pm 0.8$ & $\sqrt{ }$ & Hobbs et al. (2008) & 5.0 \\
\hline 209339 & 0.37 & 6.74 & $79.2 \pm 2.2$ & $\sqrt{ }$ & Désert et al. (1995) & 79.2 \\
\hline 216532 & 0.87 & 6.50 & $140.1 \pm 13.1$ & $\sqrt{ }$ & Désert et al. (1995) & 140.1 \\
\hline 216898 & 0.88 & 6.22 & $134.6 \pm 14.1$ & $\sqrt{ }$ & Désert et al. (1995) & 134.6 \\
\hline 217086 & 0.92 & 6.48 & $142.6 \pm 8.3$ & $\sqrt{ }$ & Désert et al. (1995) & 142.6 \\
\hline 239729 & 0.67 & 4.68 & $58.3 \pm 2.0$ & $\sqrt{ }$ & Désert et al. (1995) & 58.3 \\
\hline 242908 & 0.62 & 5.13 & $112.8 \pm 6.8$ & $\sqrt{ }$ & Désert et al. (1995) & 112.8 \\
\hline+631964 & 0.95 & 5.68 & $137.8 \pm 5.7$ & $\sqrt{ }$ & Désert et al. (1995) & 137.8 \\
\hline
\end{tabular}


Table 4. Same as Table 3 but for the $\lambda 5780 \AA$ DIB.

\begin{tabular}{|c|c|c|c|c|c|c|}
\hline $\mathrm{HD} / \mathrm{BD}$ & $\begin{array}{c}E(B-V) \\
\quad(\mathrm{mag})\end{array}$ & $\begin{array}{c}W_{2175} / E(B-V) \\
\left(\mu \mathrm{m}^{-1}\right)\end{array}$ & $\begin{array}{l}W_{5780} \\
(\mathrm{~m} \AA)\end{array}$ & $\begin{array}{c}\text { Adopt " } \sqrt{ } \text { " } \\
\text { or Reject "X" }\end{array}$ & Sources & $\begin{array}{c}W_{5780}(\mathrm{~m} \AA) \\
\text { This Work }\end{array}$ \\
\hline 2905 & 0.33 & 4.05 & $251.7 \pm 4.3$ & $\times$ & Megier et al. (2005) & 306.3 \\
\hline 2905 & 0.33 & 4.05 & 282 & $\times$ & Herbig (1993) & \\
\hline 2905 & 0.33 & 4.05 & $304 \pm 10$ & $\sqrt{ }$ & Snow et al. (2002) & \\
\hline 2905 & 0.32 & 4.05 & 288 & $x$ & Herbig (1975) & \\
\hline 2905 & 0.32 & 4.05 & 279 & $\times$ & Dorschner et al. (1977) & \\
\hline 2905 & 0.33 & 4.05 & $314 \pm 5$ & $\sqrt{ }$ & Friedman et al. (2010) & \\
\hline 2905 & 0.33 & 4.05 & 301 & $\sqrt{ }$ & Wszolk \& Godlowski (2003) & \\
\hline 15558 & 0.75 & 5.78 & $452.3 \pm 24.0$ & $\sqrt{ }$ & Désert et al. (1995) & 452.3 \\
\hline 15570 & 1.02 & 5.52 & $519.2 \pm 13.3$ & $\sqrt{ }$ & Sonnentrucher et al. (1997) & 519.2 \\
\hline 16691 & 0.83 & 6.56 & $283.9 \pm 24.9$ & $\sqrt{ }$ & Désert et al. (1995) & 283.9 \\
\hline 21291 & 0.42 & 7.31 & $191.8 \pm 5.5$ & $\sqrt{ }$ & Megier et al. (2005) & 196.5 \\
\hline 21291 & 0.43 & 7.31 & 197.8 & $\sqrt{ }$ & Cox et al. (2007) & \\
\hline 21291 & 0.41 & 7.31 & 225 & $\times$ & Herbig (1975) & \\
\hline 21291 & 0.41 & 7.31 & 200 & $\sqrt{ }$ & Wszolk \& Godlowski (2003) & \\
\hline 21483 & 0.56 & 4.07 & $181 \pm 7$ & $\sqrt{ }$ & Friedman et al. (2010) & 164.0 \\
\hline 21483 & 0.56 & 4.07 & $14 \overline{7}$ & $\sqrt{ }$ & Wszolk \& Godlowski (2003) & \\
\hline 23060 & 0.32 & 6.18 & 156 & $\sqrt{ }$ & Galazutdinov et al. (2004) & 137.5 \\
\hline 23060 & 0.34 & 6.18 & $119.0 \pm 3.7$ & $\sqrt{ }$ & Désert et al. (1995) & \\
\hline 27778 & 0.33 & 4.75 & $95.0 \pm 3.0$ & $\sqrt{ }$ & Sonnentrucher et al. (1997) & 84.0 \\
\hline 27778 & 0.37 & 4.75 & $86 \pm 4$ & $\sqrt{ }$ & Friedman et al. (2010) & \\
\hline 27778 & 0.37 & 4.75 & 71 & $\sqrt{ }$ & Wszolk \& Godlowski (2003) & \\
\hline 29647 & 1.03 & 3.82 & $51.5 \pm 3.1$ & $\sqrt{ }$ & Sonnentrucher et al. (1997) & 60.8 \\
\hline 29647 & 1.00 & 3.82 & $70 \pm 7$ & $\sqrt{ }$ & Friedman et al. (2010) & \\
\hline 30614 & 0.32 & 5.36 & $120.0 \pm 3.2$ & $\sqrt{ }$ & Sonnentrucher et al. (1997) & 121.0 \\
\hline 30614 & 0.32 & 5.36 & $111.0 \pm 17.3$ & $\sqrt{ }$ & Désert et al. (1995) & \\
\hline 30614 & 0.26 & 5.36 & $120.1 \pm 4.7$ & $\sqrt{ }$ & Megier et al. (2005) & \\
\hline 30614 & 0.30 & 5.36 & $133 \pm 5$ & $\sqrt{ }$ & Friedman et al. (2010) & \\
\hline+31643 & 0.85 & 6.59 & $259.3 \pm 9.4$ & $\sqrt{ }$ & Désert et al. (1995) & 259.3 \\
\hline 34078 & 0.49 & 6.00 & $179.0 \pm 10.8$ & $\sqrt{ }$ & Megier et al. (2005) & 182.0 \\
\hline 34078 & 0.52 & 6.00 & $137.8 \pm 16.1$ & $\times$ & Désert et al. (1995) & \\
\hline 34078 & 0.52 & 6.00 & 220 & $\times$ & Herbig (1975) & \\
\hline 34078 & 0.52 & 6.00 & $181 \pm 5$ & $\sqrt{ }$ & Friedman et al. (2010) & \\
\hline 34078 & 0.52 & 6.00 & 186 & $\sqrt{ }$ & Wszolk \& Godlowski (2003) & \\
\hline 36879 & 0.50 & 4.92 & $291.0 \pm 35.0$ & $\sqrt{ }$ & Désert et al. (1995) & 291.0 \\
\hline 37022 & 0.34 & 2.47 & 64 & $\sqrt{ }$ & Herbig (1993) & 67.3 \\
\hline 37022 & 0.36 & 2.47 & $99.7 \pm 9.0$ & $\times$ & Sonnentrucher et al. (1997) & \\
\hline 37022 & 0.34 & 2.47 & $62 \pm 8$ & $\sqrt{ }$ & Snow et al. (2002) & \\
\hline 37022 & 0.34 & 2.47 & $76 \pm 6$ & $\sqrt{ }$ & Friedman et al. (2010) & \\
\hline 37023 & 0.37 & 2.18 & 55 & $\sqrt{ }$ & Herbig (1993) & 55.0 \\
\hline 37061 & 0.56 & 3.42 & 163 & $\sqrt{ }$ & Herbig (1993) & 162.4 \\
\hline 37061 & 0.50 & 3.42 & $166.0 \pm 10.0$ & $\sqrt{ }$ & Sonnentrucher et al. (1997) & \\
\hline 37061 & 0.50 & 3.42 & $151.6 \pm 1.6$ & $\sqrt{ }$ & Megier et al. (2005) & \\
\hline 37061 & 0.52 & 3.42 & $169 \pm 7$ & $\sqrt{ }$ & Friedman et al. (2010) & \\
\hline 37367 & 0.43 & 7.15 & $417.1 \pm 58.1$ & $\sqrt{ }$ & Désert et al. (1995) & 433.4 \\
\hline 37367 & 0.40 & 7.15 & 429 & $\sqrt{ }$ & Herbig (1993) & \\
\hline 37367 & 0.40 & 7.15 & $454 \pm 5$ & $\sqrt{ }$ & Friedman et al. (2010) & \\
\hline 37903 & 0.38 & 3.64 & $145.9 \pm 23.2$ & $\sqrt{ }$ & Désert et al. (1995) & 164.0 \\
\hline 37903 & 0.35 & 3.64 & 163 & $\sqrt{ }$ & Herbig (1993) & \\
\hline 37903 & 0.35 & 3.64 & $183 \pm 10$ & $\sqrt{ }$ & Friedman et al. (2010) & \\
\hline 38087 & 0.29 & 6.03 & $162 \pm 6$ & $\sqrt{ }$ & Friedman et al. (2010) & 162.0 \\
\hline 38131 & 0.51 & 6.61 & $386.1 \pm 45.4$ & $\sqrt{ }$ & Désert et al. (1995) & 386.1 \\
\hline 40893 & 0.46 & 5.92 & $391 \pm 5$ & $\sqrt{ }$ & Friedman et al. (2010) & 391.0 \\
\hline 41117 & 0.44 & 5.89 & $329.5 \pm 4.1$ & $\sqrt{ }$ & Megier et al. (2005) & 345.5 \\
\hline 41117 & 0.46 & 5.89 & 410 & $\times$ & Dorschner et al. (1977) & \\
\hline 41117 & 0.45 & 5.89 & $356 \pm 10$ & $\sqrt{ }$ & Friedman et al. (2010) & \\
\hline 41117 & 0.45 & 5.89 & 351 & $\sqrt{ }$ & Wszolk \& Godlowski (2003) & \\
\hline 42087 & 0.35 & 6.52 & $248.1 \pm 4.1$ & $\sqrt{ }$ & Megier et al. (2005) & 259.4 \\
\hline 42087 & 0.36 & 6.52 & $275 \pm 7$ & $\sqrt{ }$ & Friedman et al. (2010) & \\
\hline 42087 & 0.36 & 6.52 & $25 \overline{5}$ & $\sqrt{ }$ & Wszolk \& Godlowski (2003) & \\
\hline 46056 & 0.50 & 5.11 & $300 \pm 7$ & $\sqrt{ }$ & Friedman et al. (2010) & 300.0 \\
\hline 46106 & 0.42 & 5.94 & 440 & $\sqrt{ }$ & Seab \& Snow (1984) & 440.0 \\
\hline 46150 & 0.46 & 5.60 & 331 & $\sqrt{ }$ & Herbig (1993) & 331.0 \\
\hline 46202 & 0.49 & 4.74 & $332 \pm 6$ & $\sqrt{ }$ & Friedman et al. (2010) & 332.0 \\
\hline 47129 & 0.34 & 6.38 & $165.5 \pm 3.7$ & $\sqrt{ }$ & Megier et al. (2005) & 166.8 \\
\hline 47129 & 0.36 & 6.38 & 250 & $x$ & Seab \& Snow (1984) & \\
\hline 47129 & 0.36 & 6.38 & $204 \pm 5$ & $x$ & Friedman et al. (2010) & \\
\hline
\end{tabular}


Table 4-Continued

\begin{tabular}{|c|c|c|c|c|c|c|}
\hline $\mathrm{HD} / \mathrm{BD}$ & $\begin{array}{c}E(B-V) \\
\quad(\mathrm{mag})\end{array}$ & $\begin{array}{c}W_{2175} / E(B-V) \\
\left(\mu \mathrm{m}^{-1}\right)\end{array}$ & $\begin{array}{l}W_{5780} \\
(\mathrm{~m} \AA)\end{array}$ & $\begin{array}{c}\text { Adopt "V" } \\
\text { or Reject "X" }\end{array}$ & Sources & $\begin{array}{l}W_{5780}(\mathrm{~m} \AA) \\
\text { This Work }\end{array}$ \\
\hline 47129 & 0.36 & 6.38 & 168 & $\checkmark$ & Wszolk \& Godlowski (2003) & \\
\hline 48099 & 0.28 & 5.63 & 199 & $\checkmark$ & Herbig (1993) & 203.0 \\
\hline 48099 & 0.27 & 5.63 & 250 & $\times$ & Seab \& Snow (1984) & \\
\hline 48099 & 0.27 & 5.63 & $207 \pm 7$ & $\checkmark$ & Friedman et al. (2010) & \\
\hline 48434 & 0.28 & 6.31 & 270 & $\checkmark$ & Seab \& Snow (1984) & 270.0 \\
\hline+60497 & 0.89 & 5.94 & $395.2 \pm 26.7$ & $\sqrt{ }$ & Désert et al. (1995) & 395.2 \\
\hline+60594 & 0.62 & 6.23 & 285 & $\sqrt{ }$ & Galazutdinov et al. (2004) & 285.0 \\
\hline 122879 & 0.35 & 6.83 & 311.1 & $\checkmark$ & Benvenuti \& Porceddu (1989) & 292.7 \\
\hline 122879 & 0.34 & 6.83 & $274.2 \pm 4.6$ & $\sqrt{ }$ & Megier et al. (2005) & \\
\hline 123008 & 0.63 & 4.94 & 495.8 & $\checkmark$ & Benvenuti \& Porceddu (1989) & 495.8 \\
\hline 142096 & 0.19 & 4.46 & $83.1 \pm 1.6$ & $\checkmark$ & Megier et al. (2005) & 83.1 \\
\hline 143018 & 0.05 & 5.12 & $39 \pm 4$ & $\checkmark$ & Friedman et al. (2010) & 39.0 \\
\hline 143275 & 0.17 & 5.27 & $82 \pm 5$ & $\sqrt{ }$ & Friedman et al. (2010) & 80.0 \\
\hline 143275 & 0.17 & 5.27 & 78 & $\sqrt{ }$ & Wszolk \& Godlowski (2003) & \\
\hline 144217 & 0.17 & 6.49 & 148.5 & $\sqrt{ }$ & Galazutdinov et al. (2004) & 164.9 \\
\hline 144217 & 0.19 & 6.49 & $183.0 \pm 8.0$ & $\checkmark$ & Sonnentrucher et al. (1997) & \\
\hline 144217 & 0.20 & 6.49 & 250 & $\times$ & Dorschner et al. (1977) & \\
\hline 144217 & 0.19 & 6.50 & $171 \pm 5$ & $\checkmark$ & Friedman et al. (2010) & \\
\hline 144217 & 0.19 & 6.50 & 157 & $\sqrt{ }$ & Wszolk \& Godlowski (2003) & \\
\hline 144470 & 0.22 & 6.62 & 184 & $\sqrt{ }$ & Herbig (1993) & 180.0 \\
\hline 144470 & 0.19 & 6.62 & $173.8 \pm 1.9$ & $\checkmark$ & Megier et al. (2005) & \\
\hline 144470 & 0.19 & 6.62 & 178 & $\checkmark$ & Benvenuti \& Porceddu (1989) & \\
\hline 144470 & 0.22 & 6.62 & 201 & $\times$ & Herbig (1975) & \\
\hline 144470 & 0.22 & 6.62 & 201 & $x$ & Dorschner et al. (1977) & \\
\hline 144470 & 0.22 & 6.62 & $192 \pm 5$ & $\checkmark$ & Friedman et al. (2010) & \\
\hline 144470 & 0.22 & 6.62 & 172 & $\checkmark$ & Wszolk \& Godlowski (2003) & \\
\hline 145502 & 0.25 & 6.25 & 182 & $\checkmark$ & Herbig (1993) & 184.5 \\
\hline 145502 & 0.24 & 6.25 & $210.0 \pm 8.4$ & $x$ & Sonnentrucher et al. (1997) & \\
\hline 145502 & 0.24 & 6.25 & $187 \pm 5$ & $\sqrt{ }$ & Friedman et al. (2010) & \\
\hline 145502 & 0.24 & 6.25 & 164 & $x$ & Wszolk \& Godlowski (2003) & \\
\hline 147165 & 0.34 & 5.27 & $243.3 \pm 3.1$ & $\hat{v}$ & Megier et al. (2005) & 224.8 \\
\hline 147165 & 0.39 & 5.27 & 301 & $\times$ & Herbig (1975) & \\
\hline 147165 & 0.40 & 5.27 & 340 & $x$ & Dorschner et al. (1977) & \\
\hline 147165 & 0.41 & 5.27 & $254 \pm 5$ & $\checkmark$ & Friedman et al. (2010) & \\
\hline 147165 & 0.41 & 5.27 & 237 & $\checkmark$ & Wszolk \& Godlowski (2003) & \\
\hline 147888 & 0.47 & 5.87 & $254 \pm 5$ & $\sqrt{ }$ & Snow et al. (2002) & 253.0 \\
\hline 147888 & 0.47 & 5.87 & $252 \pm 12$ & $\checkmark$ & Friedman et al. (2010) & \\
\hline 147889 & 1.08 & 6.88 & 430 & $x$ & Herbig (1975) & 370.5 \\
\hline 147889 & 1.08 & 6.88 & 379 & $\checkmark$ & Herbig (1993) & \\
\hline 147889 & 1.10 & 6.88 & 379 & $\checkmark$ & Herbig (2000) & \\
\hline 147889 & 1.08 & 6.88 & 430 & $\times$ & Seab \& Snow (1984) & \\
\hline 147889 & 1.07 & 6.88 & $377 \pm 8$ & $\sqrt{ }$ & Friedman et al. (2010) & \\
\hline 147889 & 1.07 & 6.88 & 347 & $\sqrt{ }$ & Wszolk \& Godlowski (2003) & \\
\hline 147933 & 0.47 & 5.62 & 258 & $x$ & Herbig (1975) & 212.3 \\
\hline 147933 & 0.46 & 5.62 & 219 & $\checkmark$ & Herbig (1993) & \\
\hline 147933 & 0.45 & 5.62 & $208.0 \pm 12.5$ & $\checkmark$ & Megier et al. (2005) & \\
\hline 147933 & 0.46 & 5.62 & 280 & $x$ & Seab \& Snow (1984) & \\
\hline 147933 & 0.46 & 5.62 & 292 & $x$ & Dorschner et al. (1977) & \\
\hline 147933 & 0.48 & 5.62 & $222 \pm 10$ & $\checkmark$ & Friedman et al. (2010) & \\
\hline 147933 & 0.48 & 5.62 & 200 & $\checkmark$ & Wszolk \& Godlowski (2003) & \\
\hline 149757 & 0.32 & 6.63 & 72 & $\sqrt{ }$ & Herbig (1993) & 71.8 \\
\hline 149757 & 0.32 & 6.63 & 250 & $\times$ & Dorschner et al. (1977) & \\
\hline 149757 & 0.29 & 6.63 & 66.4 & $\sqrt{ }$ & Galazutdinov et al. (2004) & \\
\hline 149757 & 0.29 & 6.63 & $66.4 \pm 1.9$ & $\sqrt{ }$ & Megier et al. (2005) & \\
\hline 149757 & 0.32 & 6.63 & $78.1 \pm 1.9$ & $\sqrt{ }$ & Sonnentrucher et al. (1997) & \\
\hline 149757 & 0.32 & 6.63 & $83 \pm 7$ & $\sqrt{ }$ & Friedman et al. (2010) & \\
\hline 149757 & 0.32 & 6.63 & 65 & $\checkmark$ & Wszolk \& Godlowski (2003) & \\
\hline 152233 & 0.45 & 5.76 & 158.9 & $\sqrt{ }$ & Benvenuti \& Porceddu (1989) & 158.9 \\
\hline 152247 & 0.44 & 5.80 & 224 & $\checkmark$ & Benvenuti \& Porceddu (1989) & 224.0 \\
\hline 152248 & 0.45 & 5.24 & 228.1 & $\checkmark$ & Benvenuti \& Porceddu (1989) & 228.1 \\
\hline 152249 & 0.46 & 6.13 & 257.1 & $\checkmark$ & Benvenuti \& Porceddu (1989) & 257.1 \\
\hline 154445 & 0.43 & 7.34 & $163.8 \pm 26.7$ & $\times$ & Désert et al. (1995) & 198.9 \\
\hline 154445 & 0.39 & 7.34 & $192.6 \pm 7.0$ & $\checkmark$ & Megier et al. (2005) & \\
\hline 154445 & 0.42 & 7.34 & $212.1 \pm 5.0$ & $\checkmark$ & Sonnentrucher et al. (1997) & \\
\hline 154445 & 0.42 & 7.34 & 192 & $\checkmark$ & Wszolk \& Godlowski (2003) & \\
\hline 162978 & 0.35 & 5.32 & 209 & $\checkmark$ & Benvenuti \& Porceddu (1989) & 210.0 \\
\hline
\end{tabular}


Table 4-Continued

\begin{tabular}{|c|c|c|c|c|c|c|}
\hline $\mathrm{HD} / \mathrm{BD}$ & $\begin{array}{c}E(B-V) \\
(\mathrm{mag})\end{array}$ & $\begin{array}{c}W_{2175} / E(B-V) \\
\left(\mu \mathrm{m}^{-1}\right)\end{array}$ & $\begin{array}{c}W_{5780} \\
(\mathrm{~m} \AA)\end{array}$ & $\begin{array}{c}\text { Adopt " } \sqrt{ } \text { " } \\
\text { or Reject "X" }\end{array}$ & Sources & $\begin{array}{c}W_{5780}(\mathrm{~m} \AA) \\
\text { This Work }\end{array}$ \\
\hline 162978 & 0.35 & 5.32 & $211 \pm 14$ & $\sqrt{ }$ & Friedman et al. (2010) & \\
\hline 164492 & 0.32 & 6.67 & 226 & $x$ & Herbig (2000) & 226.0 \\
\hline 164794 & 0.33 & 6.31 & 161 & $\sqrt{ }$ & Benvenuti \& Porceddu (1989) & 161.0 \\
\hline 165052 & 0.44 & 5.68 & 211.2 & $\sqrt{ }$ & Benvenuti \& Porceddu (1989) & 211.2 \\
\hline 166937 & 0.24 & 6.23 & 231 & $\times$ & Herbig $(1975)$ & 279.0 \\
\hline 166937 & 0.26 & 6.23 & $277.1 \pm 2.8$ & $\sqrt{ }$ & Megier et al. (2005) & \\
\hline 166937 & 0.51 & 6.23 & $242.8 \pm 24.0$ & $\times$ & Désert et al. (1995) & \\
\hline 166937 & 0.25 & 6.23 & 279 & $\sqrt{ }$ & Benvenuti \& Porceddu (1989) & \\
\hline 166937 & 0.25 & 6.23 & $283 \pm 4$ & $\sqrt{ }$ & Friedman et al. (2010) & \\
\hline 166937 & 0.25 & 6.23 & 277 & $\sqrt{ }$ & Wszolk \& Godlowski (2003) & \\
\hline 167971 & 1.05 & 5.46 & $539.2 \pm 12.6$ & $\sqrt{ }$ & Megier et al. (2005) & 525.6 \\
\hline 167971 & 1.08 & 5.46 & 580 & $\times$ & Herbig (1975) & \\
\hline 167971 & 1.08 & 5.46 & $512 \pm 9$ & $\sqrt{ }$ & Friedman et al. (2010) & \\
\hline 168076 & 0.80 & 4.81 & $372.0 \pm 28.8$ & $\times$ & Désert et al. (1995) & 513.1 \\
\hline 168076 & 0.81 & 4.81 & 485.2 & $\sqrt{ }$ & Benvenuti \& Porceddu (1989) & \\
\hline 168076 & 0.78 & 4.81 & $541 \pm 10$ & $\sqrt{ }$ & Friedman et al. (2010) & \\
\hline 168112 & 1.04 & 5.44 & $435.8 \pm 38.5$ & $\sqrt{ }$ & Désert et al. (1995) & 490.4 \\
\hline 168112 & 1.01 & 5.44 & 545 & $\sqrt{ }$ & Herbig (2000) & \\
\hline 183143 & 1.24 & 6.13 & $720.4 \pm 47.1$ & $\sqrt{ }$ & Désert et al. (1995) & 760.5 \\
\hline 183143 & 1.28 & 6.13 & 755.2 & $\sqrt{ }$ & Cox et al. (2007) & \\
\hline 183143 & 1.27 & 6.13 & $751 \pm 10$ & $\sqrt{ }$ & Snow et al. (2002) & \\
\hline 183143 & 1.28 & 6.13 & $760.3 \pm 17.9$ & $\sqrt{ }$ & Sonnentrucher et al. (1997) & \\
\hline 183143 & 1.28 & 6.13 & 774 & $\sqrt{ }$ & Herbig (1993) & \\
\hline 183143 & 1.28 & 6.13 & 660 & $\times$ & Seab \& Snow (1984) & \\
\hline 183143 & 1.27 & 6.13 & $761 \pm 6$ & $\sqrt{ }$ & Friedman et al. (2010) & \\
\hline 183143 & 1.27 & 6.13 & $779.3 \pm 2.3$ & $\sqrt{ }$ & Hobbs et al. (2009) & \\
\hline 183143 & 1.27 & 6.13 & 783 & $\sqrt{ }$ & Wszolk \& Godlowski (2003) & \\
\hline 185418 & 0.50 & 6.67 & $273 \pm 5$ & $\sqrt{ }$ & Friedman et al. (2010) & 273.0 \\
\hline 190603 & 0.72 & 4.88 & $424.8 \pm 11.5$ & $\times$ & Sonnentrucher et al. (1997) & 341.5 \\
\hline 190603 & 0.71 & 4.88 & $356.8 \pm 11.1$ & $\sqrt{ }$ & Megier et al. (2005) & \\
\hline 190603 & 0.71 & 4.88 & 534 & $x$ & Herbig (1975) & \\
\hline 190603 & 0.94 & 4.88 & $326.2 \pm 7.5$ & $\sqrt{ }$ & Désert et al. (1995) & \\
\hline 192281 & 0.73 & 5.78 & $268.6 \pm 25.6$ & $\sqrt{ }$ & Désert et al. (1995) & 268.6 \\
\hline 193322 & 0.41 & 4.15 & 194 & $\sqrt{ }$ & Herbig (1993) & 180.5 \\
\hline 193322 & 0.41 & 4.15 & 167 & $\sqrt{ }$ & Wszolk \& Godlowski (2003) & \\
\hline 193682 & 0.83 & 7.40 & $376.0 \pm 27.4$ & $\sqrt{ }$ & Désert et al. (1995) & 376.0 \\
\hline 197770 & 0.58 & 6.82 & 146.7 & $\sqrt{ }$ & Cox et al. (2007) & 146.7 \\
\hline 198478 & 0.54 & 5.44 & 313.7 & $\sqrt{ }$ & Cox et al. (2007) & 316.2 \\
\hline 198478 & 0.54 & 5.44 & $305.0 \pm 15.0$ & $\sqrt{ }$ & Megier et al. (2005) & \\
\hline 198478 & 0.54 & 5.44 & $253.8 \pm 9.7$ & $\times$ & Sonnentrucher et al. (1997) & \\
\hline 198478 & 0.53 & 5.44 & 399 & $x$ & Herbig (1975) & \\
\hline 198478 & 0.53 & 5.44 & 410 & $x$ & Dorschner et al. (1977) & \\
\hline 198478 & 0.54 & 5.44 & $332 \pm 5$ & $\sqrt{ }$ & Friedman et al. (2010) & \\
\hline 198478 & 0.54 & 5.44 & 314 & $\sqrt{ }$ & Wszolk \& Godlowski (2003) & \\
\hline 199216 & 0.73 & 5.89 & $186.2 \pm 4.4$ & $\sqrt{ }$ & Désert et al. (1995) & 186.2 \\
\hline 199478 & 0.47 & 6.42 & 490 & $\sqrt{ }$ & Herbig (1975) & 490.0 \\
\hline 199579 & 0.38 & 4.60 & $104.9 \pm 13.7$ & $\sqrt{ }$ & Désert et al. (1995) & 123.8 \\
\hline 199579 & 0.38 & 4.60 & 136 & $\sqrt{ }$ & Herbig (1993) & \\
\hline 199579 & 0.35 & 4.60 & $127.0 \pm 8.5$ & $\sqrt{ }$ & Megier et al. (2005) & \\
\hline 199579 & 0.37 & 4.60 & 136 & $\sqrt{ }$ & Herbig (2000) & \\
\hline 199579 & 0.37 & 4.60 & 240 & $\times$ & Seab \& Snow (1984) & \\
\hline 199579 & 0.36 & 4.60 & 236 & $\times$ & Herbig (1975) & \\
\hline 199579 & 0.37 & 4.60 & $128 \pm 5$ & $\sqrt{ }$ & Friedman et al. (2010) & \\
\hline 199579 & 0.37 & 4.60 & 111 & $\sqrt{ }$ & Wszolk \& Godlowski (2003) & \\
\hline 203938 & 0.66 & 5.70 & 320 & $\sqrt{ }$ & Galazutdinov et al. (2004) & 332.0 \\
\hline 203938 & 0.70 & 5.70 & $320.0 \pm 6.5$ & $\sqrt{ }$ & Megier et al. (2005) & \\
\hline 203938 & 0.74 & 5.70 & $356 \pm 5$ & $\sqrt{ }$ & Friedman et al. (2010) & \\
\hline 204827 & 1.06 & 4.25 & 227 & $\sqrt{ }$ & Galazutdinov et al. (2004) & 247.0 \\
\hline 204827 & 1.11 & 4.25 & $257 \pm 4$ & $\sqrt{ }$ & Friedman et al. (2010) & \\
\hline 204827 & 1.11 & 4.25 & $257.0 \pm 3.0$ & $\sqrt{ }$ & Hobbs et al. (2008) & \\
\hline 206165 & 0.47 & 6.07 & $168.3 \pm 8.0$ & $\times$ & Sonnentrucher et al. (1997) & 209.0 \\
\hline 206165 & 0.48 & 6.07 & 197 & $\sqrt{ }$ & Herbig (1993) & \\
\hline 206165 & 0.46 & 6.07 & $204.0 \pm 2.2$ & $\sqrt{ }$ & Megier et al. (2005) & \\
\hline 206165 & 0.46 & 6.07 & 350 & $\times$ & Dorschner et al. (1977) & \\
\hline 206165 & 0.47 & 6.07 & $231 \pm 7$ & $\sqrt{ }$ & Friedman et al. (2010) & \\
\hline 206165 & 0.47 & 6.07 & 204 & $\sqrt{ }$ & Wszolk \& Godlowski (2003) & \\
\hline
\end{tabular}


Table 4-Continued

\begin{tabular}{|c|c|c|c|c|c|c|}
\hline $\mathrm{HD} / \mathrm{BD}$ & $\begin{array}{c}E(B-V) \\
\quad(\mathrm{mag})\end{array}$ & $\begin{array}{c}W_{2175} / E(B-V) \\
\left(\mu \mathrm{m}^{-1}\right)\end{array}$ & $\begin{array}{l}W_{5780} \\
(\mathrm{~m} \AA)\end{array}$ & $\begin{array}{c}\text { Adopt " } \sqrt{ } \text { " } \\
\text { or Reject "X" }\end{array}$ & Sources & $\begin{array}{c}W_{5780}(\mathrm{~m} \AA) \\
\text { This Work }\end{array}$ \\
\hline 206267 & 0.50 & 4.96 & $222.7 \pm 3.6$ & $\sqrt{ }$ & Megier et al. (2005) & 231.5 \\
\hline 206267 & 0.49 & 4.96 & 223.2 & $\sqrt{ }$ & Galazutdinov et al. (2004) & \\
\hline 206267 & 0.53 & 4.96 & $242 \pm 7$ & $\sqrt{ }$ & Friedman et al. (2010) & \\
\hline 206267 & 0.53 & 4.96 & 238 & $\sqrt{ }$ & Wszolk \& Godlowski (2003) & \\
\hline 207198 & 0.62 & 5.23 & 242 & $\sqrt{ }$ & Herbig (1993) & 246.2 \\
\hline 207198 & 0.54 & 5.23 & $237.5 \pm 4.5$ & $\sqrt{ }$ & Megier et al. (2005) & \\
\hline 207198 & 0.56 & 5.23 & 237.5 & $\sqrt{ }$ & Galazutdinov et al. (2004) & \\
\hline 207198 & 0.62 & 5.23 & $262 \pm 6$ & $\sqrt{ }$ & Friedman et al. (2010) & \\
\hline 207198 & 0.62 & 5.23 & 252 & $\sqrt{ }$ & Wszolk \& Godlowski (2003) & \\
\hline 209339 & 0.37 & 6.74 & $220.9 \pm 5.9$ & $\sqrt{ }$ & Désert et al. (1995) & 220.9 \\
\hline 209339 & 0.36 & 6.74 & 350 & $\times$ & Seab \& Snow (1984) & \\
\hline 210121 & 0.40 & 2.74 & $70 \pm 7$ & $\sqrt{ }$ & Friedman et al. (2010) & 70.0 \\
\hline 216532 & 0.87 & 6.50 & $430.7 \pm 40.0$ & $\sqrt{ }$ & Désert et al. (1995) & 430.7 \\
\hline 216898 & 0.88 & 6.22 & $440.0 \pm 44.9$ & $\sqrt{ }$ & Désert et al. (1995) & 440.0 \\
\hline 217086 & 0.92 & 6.48 & $495.0 \pm 26.7$ & $\sqrt{ }$ & Désert et al. (1995) & 513.5 \\
\hline 217086 & 0.92 & 6.48 & 532 & $\sqrt{ }$ & Galazutdinov et al. (2004) & \\
\hline 229196 & 1.22 & 5.51 & 481 & $\sqrt{ }$ & Herbig (2000) & 481.0 \\
\hline 239729 & 0.67 & 4.68 & $186.9 \pm 3.4$ & $\sqrt{ }$ & Désert et al. (1995) & 168.9 \\
\hline 242908 & 0.62 & 5.13 & $355.9 \pm 18.0$ & $\sqrt{ }$ & Désert et al. (1995) & 355.9 \\
\hline 303308 & 0.46 & 4.86 & 264 & $\sqrt{ }$ & Benvenuti \& Porceddu (1989) & 264.0 \\
\hline+631964 & 0.95 & 5.68 & $648.9 \pm 14.3$ & $\sqrt{ }$ & Désert et al. (1995) & 645.1 \\
\hline+631964 & 0.96 & 5.68 & $641.2 \pm 7.3$ & $\sqrt{ }$ & Megier et al. (2005) & \\
\hline+631964 & 1.00 & 5.68 & $729 \pm 10$ & $\times$ & Friedman et al. (2010) & \\
\hline
\end{tabular}


Table 5. Same as Table 3 but for the $\lambda 5797 \AA$ DIB.

\begin{tabular}{|c|c|c|c|c|c|c|}
\hline $\mathrm{HD} / \mathrm{BD}$ & $\begin{array}{c}E(B-V) \\
\quad(\mathrm{mag})\end{array}$ & $\begin{array}{c}W_{2175} / E(B-V) \\
\left(\mu \mathrm{m}^{-1}\right)\end{array}$ & $\begin{array}{c}W_{5797} \\
(\mathrm{~m} \AA)\end{array}$ & $\begin{array}{l}\text { Adopt " } \sqrt{ } \text { " } \\
\text { or Reject "X" }\end{array}$ & Sources & $\begin{array}{c}W_{5797}(\mathrm{~m} \AA) \\
\text { This Work }\end{array}$ \\
\hline 2905 & 0.33 & 4.05 & $68.9 \pm 2.1$ & $\sqrt{ }$ & Megier et al. (2005) & 75.7 \\
\hline 2905 & 0.33 & 4.05 & 72 & $\sqrt{ }$ & Herbig (1993) & \\
\hline 2905 & 0.33 & 4.05 & $74 \pm 4$ & $\sqrt{ }$ & Snow et al. (2002) & \\
\hline 2905 & 0.32 & 4.05 & 114 & $\times$ & Herbig (1975) & \\
\hline 2905 & 0.32 & 4.05 & 147 & $\times$ & Dorschner et al. (1977) & \\
\hline 2905 & 0.33 & 4.05 & $110 \pm 5$ & $x$ & Friedman et al. (2010) & \\
\hline 2905 & 0.33 & 4.05 & 88 & $\sqrt{ }$ & Wszolk \& Godlowski (2003) & \\
\hline 15558 & 0.75 & 5.78 & $129.0 \pm 7.5$ & $\sqrt{ }$ & Désert et al. (1995) & 129.0 \\
\hline 15570 & 1.02 & 5.52 & $161.2 \pm 9.2$ & $\sqrt{ }$ & Sonnentrucher et al. (1997) & 161.2 \\
\hline 16691 & 0.83 & 6.56 & $63.9 \pm 6.6$ & $\sqrt{ }$ & Désert et al. (1995) & 63.9 \\
\hline 21291 & 0.42 & 7.31 & $48.6 \pm 2.1$ & $\times$ & Megier et al. (2005) & 72.5 \\
\hline 21291 & 0.43 & 7.31 & 71.0 & $\sqrt{ }$ & Cox et al. (2007) & \\
\hline 21291 & 0.41 & 7.31 & 135 & $\times$ & Herbig (1975) & \\
\hline 21291 & 0.41 & 7.31 & 74 & $\sqrt{ }$ & Wszolk \& Godlowski (2003) & \\
\hline 21483 & 0.56 & 4.07 & $96 \pm 6$ & $\sqrt{ }$ & Friedman et al. (2010) & 89.0 \\
\hline 21483 & 0.56 & 4.07 & 82 & $\sqrt{ }$ & Wszolk \& Godlowski (2003) & \\
\hline 23060 & 0.32 & 6.18 & 35 & $\sqrt{ }$ & Galazutdinov et al. (2004) & 42.0 \\
\hline 23060 & 0.34 & 6.18 & $49.0 \pm 1.7$ & $\sqrt{ }$ & Désert et al. (1995) & \\
\hline 27778 & 0.33 & 4.75 & $31.0 \pm 2.0$ & $\sqrt{ }$ & Sonnentrucher et al. (1997) & 36.7 \\
\hline 27778 & 0.37 & 4.75 & $39 \pm 2$ & $\sqrt{ }$ & Friedman et al. (2010) & \\
\hline 27778 & 0.37 & 4.75 & 40 & $\sqrt{ }$ & Wszolk \& Godlowski (2003) & \\
\hline 29647 & 1.03 & 3.82 & $33.0 \pm 8.2$ & $\sqrt{ }$ & Sonnentrucher et al. (1997) & 36.0 \\
\hline 29647 & 1.00 & 3.82 & $39 \pm 5$ & $\sqrt{ }$ & Friedman et al. (2010) & \\
\hline 30614 & 0.32 & 5.36 & $33.0 \pm 5.1$ & $\sqrt{ }$ & Sonnentrucher et al. (1997) & 45.3 \\
\hline 30614 & 0.32 & 5.36 & $45.1 \pm 7.0$ & $\sqrt{ }$ & Désert et al. (1995) & \\
\hline 30614 & 0.26 & 5.36 & $47.1 \pm 2.1$ & $\sqrt{ }$ & Megier et al. (2005) & \\
\hline 30614 & 0.30 & 5.36 & $56 \pm 3$ & $\sqrt{ }$ & Friedman et al. (2010) & \\
\hline+31643 & 0.85 & 6.59 & $78.2 \pm 3.4$ & $\sqrt{ }$ & Désert et al. (1995) & 78.2 \\
\hline 34078 & 0.49 & 6.00 & $56.1 \pm 3.7$ & $\sqrt{ }$ & Megier et al. (2005) & 55.6 \\
\hline 34078 & 0.52 & 6.00 & $43.2 \pm 5.2$ & $\sqrt{ }$ & Désert et al. (1995) & \\
\hline 34078 & 0.52 & 6.00 & 172 & $\times$ & Herbig (1975) & \\
\hline 34078 & 0.52 & 6.00 & $56 \pm 3$ & $\sqrt{ }$ & Friedman et al. (2010) & \\
\hline 34078 & 0.52 & 6.00 & 67 & $\sqrt{ }$ & Wszolk \& Godlowski (2003) & \\
\hline 36879 & 0.50 & 4.92 & $53.0 \pm 6.5$ & $\sqrt{ }$ & Désert et al. (1995) & 53.0 \\
\hline 37022 & 0.34 & 2.47 & 3 & $\times$ & Herbig (1993) & 15.1 \\
\hline 37022 & 0.36 & 2.47 & $11.2 \pm 5.0$ & $\sqrt{ }$ & Sonnentrucher et al. (1997) & \\
\hline 37022 & 0.34 & 2.47 & $19 \pm 6$ & $\sqrt{ }$ & Friedman et al. (2010) & \\
\hline 37023 & 0.37 & 2.18 & 16 & $\sqrt{ }$ & Herbig (1993) & 16.0 \\
\hline 37061 & 0.56 & 3.42 & 40 & $\sqrt{ }$ & Herbig (1993) & 33.3 \\
\hline 37061 & 0.50 & 3.42 & $25.0 \pm 9.0$ & $\sqrt{ }$ & Sonnentrucher et al. (1997) & \\
\hline 37061 & 0.50 & 3.42 & $10.5 \pm 1.9$ & $\times$ & Megier et al. (2005) & \\
\hline 37061 & 0.52 & 3.42 & $35 \pm 5$ & $\sqrt{ }$ & Friedman et al. (2010) & \\
\hline 37367 & 0.43 & 7.15 & $85.1 \pm 12.0$ & $\sqrt{ }$ & Désert et al. (1995) & 108.7 \\
\hline 37367 & 0.40 & 7.15 & 108 & $\sqrt{ }$ & Herbig (1993) & \\
\hline 37367 & 0.40 & 7.15 & $133 \pm 6$ & $\sqrt{ }$ & Friedman et al. (2010) & \\
\hline 37903 & 0.38 & 3.64 & $55.1 \pm 8.7$ & $\times$ & Désert et al. (1995) & 28.5 \\
\hline 37903 & 0.35 & 3.64 & 24 & $\sqrt{ }$ & Herbig (1993) & \\
\hline 37903 & 0.35 & 3.64 & $33 \pm 5$ & $\sqrt{ }$ & Friedman et al. (2010) & \\
\hline 38087 & 0.29 & 6.03 & $44 \pm 6$ & $\sqrt{ }$ & Friedman et al. (2010) & 44.0 \\
\hline 38131 & 0.51 & 6.61 & $85.2 \pm 10.2$ & $\sqrt{ }$ & Désert et al. (1995) & 85.2 \\
\hline 40893 & 0.46 & 5.92 & $109 \pm 6$ & $\sqrt{ }$ & Friedman et al. (2010) & 109.0 \\
\hline 41117 & 0.44 & 5.89 & $122.7 \pm 2.0$ & $\sqrt{ }$ & Megier et al. (2005) & 132.6 \\
\hline 41117 & 0.46 & 5.89 & 180 & $\times$ & Dorschner et al. (1977) & \\
\hline 41117 & 0.45 & 5.89 & $148 \pm 8$ & $\sqrt{ }$ & Friedman et al. (2010) & \\
\hline 41117 & 0.45 & 5.89 & 127 & $\sqrt{ }$ & Wszolk \& Godlowski (2003) & \\
\hline 42087 & 0.35 & 6.52 & $91.7 \pm 1.8$ & $\sqrt{ }$ & Megier et al. (2005) & 96.9 \\
\hline 42087 & 0.36 & 6.52 & $99 \pm 2$ & $\sqrt{ }$ & Friedman et al. (2010) & \\
\hline 42087 & 0.36 & 6.52 & 100 & $\sqrt{ }$ & Wszolk \& Godlowski (2003) & \\
\hline 46056 & 0.50 & 5.11 & $135 \pm 9$ & $\sqrt{ }$ & Friedman et al. (2010) & 135.0 \\
\hline 46150 & 0.46 & 5.60 & 102 & $\sqrt{ }$ & Herbig (1993) & 102.0 \\
\hline 46202 & 0.49 & 4.74 & $119 \pm 8$ & $\sqrt{ }$ & Friedman et al. (2010) & 119.0 \\
\hline 47129 & 0.34 & 6.38 & $40.1 \pm 1.6$ & $\sqrt{ }$ & Megier et al. (2005) & 64.4 \\
\hline 47129 & 0.36 & 6.38 & $89 \pm 4$ & $\sqrt{ }$ & Friedman et al. (2010) & \\
\hline 47129 & 0.36 & 6.38 & 64 & $\sqrt{ }$ & Wszolk \& Godlowski (2003) & \\
\hline 48099 & 0.28 & 5.63 & 42 & $\sqrt{ }$ & Herbig (1993) & 47.0 \\
\hline 48099 & 0.27 & 5.63 & $52 \pm 6$ & $\sqrt{ }$ & Friedman et al. (2010) & \\
\hline
\end{tabular}


Table 5-Continued

\begin{tabular}{|c|c|c|c|c|c|c|}
\hline $\mathrm{HD} / \mathrm{BD}$ & $\begin{array}{c}E(B-V) \\
(\mathrm{mag})\end{array}$ & $\begin{array}{c}W_{2175} / E(B-V) \\
\left(\mu \mathrm{m}^{-1}\right)\end{array}$ & $\begin{array}{c}W_{5797} \\
(\mathrm{~m} \AA) \\
\end{array}$ & $\begin{array}{c}\text { Adopt " } \sqrt{ } \text { " } \\
\text { or Reject "X" }\end{array}$ & Sources & $\begin{array}{c}W_{5797}(\mathrm{~m} \AA) \\
\text { This Work }\end{array}$ \\
\hline+60497 & 0.89 & 5.94 & $121.9 \pm 8.9$ & $\sqrt{ }$ & Désert et al. (1995) & 121.9 \\
\hline+60594 & 0.62 & 6.23 & 98 & $\sqrt{ }$ & Galazutdinov et al. (2004) & 98.0 \\
\hline 122879 & 0.35 & 6.83 & 74.9 & $\sqrt{ }$ & Benvenuti \& Porceddu (1989) & 60.1 \\
\hline 122879 & 0.34 & 6.83 & $45.3 \pm 1.7$ & $\sqrt{ }$ & Megier et al. (2005) & \\
\hline 123008 & 0.63 & 4.94 & 127.3 & $\sqrt{ }$ & Benvenuti \& Porceddu (1989) & 127.3 \\
\hline 142096 & 0.19 & 4.46 & $12.3 \pm 0.6$ & $\sqrt{ }$ & Megier et al. (2005) & 12.0 \\
\hline 143018 & 0.05 & 5.12 & $7 \pm 2$ & $\sqrt{ }$ & Friedman et al. (2010) & 7.0 \\
\hline 143275 & 0.17 & 5.27 & $26 \pm 4$ & $\sqrt{ }$ & Friedman et al. (2010) & 21.5 \\
\hline 143275 & 0.17 & 5.27 & 17 & $\sqrt{ }$ & Wszolk \& Godlowski (2003) & \\
\hline 144217 & 0.17 & 6.49 & 18.6 & $\sqrt{ }$ & Galazutdinov et al. (2004) & 15.8 \\
\hline 144217 & 0.19 & 6.49 & $10.8 \pm 1.1$ & $\sqrt{ }$ & Sonnentrucher et al. (1997) & \\
\hline 144217 & 0.20 & 6.49 & 110 & $x$ & Dorschner et al. (1977) & \\
\hline 144217 & 0.19 & 6.50 & $34 \pm 4$ & $\times$ & Friedman et al. (2010) & \\
\hline 144217 & 0.19 & 6.50 & 18 & $\sqrt{ }$ & Wszolk \& Godlowski (2003) & \\
\hline 144470 & 0.22 & 6.62 & 34 & $\sqrt{ }$ & Herbig (1993) & 41.0 \\
\hline 144470 & 0.19 & 6.62 & $22.8 \pm 0.6$ & $\times$ & Megier et al. (2005) & \\
\hline 144470 & 0.19 & 6.62 & 51.9 & $\sqrt{ }$ & Benvenuti \& Porceddu (1989) & \\
\hline 144470 & 0.22 & 6.62 & 45 & $\sqrt{ }$ & Herbig (1975) & \\
\hline 144470 & 0.22 & 6.62 & 45 & $\sqrt{ }$ & Dorschner et al. (1977) & \\
\hline 144470 & 0.22 & 6.62 & $40 \pm 4$ & $\sqrt{ }$ & Friedman et al. (2010) & \\
\hline 144470 & 0.22 & 6.62 & 30 & $\sqrt{ }$ & Wszolk \& Godlowski (2003) & \\
\hline 145502 & 0.25 & 6.25 & 41 & $\sqrt{ }$ & Herbig (1993) & 40.5 \\
\hline 145502 & 0.24 & 6.25 & $34.1 \pm 10.8$ & $\sqrt{ }$ & Sonnentrucher et al. (1997) & \\
\hline 145502 & 0.24 & 6.25 & $49 \pm 5$ & $\sqrt{ }$ & Friedman et al. (2010) & \\
\hline 145502 & 0.24 & 6.25 & 38 & $\sqrt{ }$ & Wszolk \& Godlowski (2003) & \\
\hline 147165 & 0.34 & 5.27 & $26.3 \pm 4.9$ & $\sqrt{ }$ & Megier et al. (2005) & 37.4 \\
\hline 147165 & 0.39 & 5.27 & 91 & $\times$ & Herbig (1975) & \\
\hline 147165 & 0.40 & 5.27 & 105 & $\times$ & Dorschner et al. (1977) & \\
\hline 147165 & 0.41 & 5.27 & $54 \pm 3$ & $\sqrt{ }$ & Friedman et al. (2010) & \\
\hline 147165 & 0.41 & 5.27 & 32 & $\sqrt{ }$ & Wszolk \& Godlowski (2003) & \\
\hline 147888 & 0.47 & 5.87 & $55 \pm 3$ & $\sqrt{ }$ & Snow et al. (2002) & 57.5 \\
\hline 147888 & 0.47 & 5.87 & $60 \pm 5$ & $\sqrt{ }$ & Friedman et al. (2010) & \\
\hline 147889 & 1.08 & 6.88 & 150 & $\sqrt{ }$ & Herbig (1975) & 160.0 \\
\hline 147889 & 1.08 & 6.88 & 173 & $\sqrt{ }$ & Herbig (1993) & \\
\hline 147889 & 1.07 & 6.88 & $163 \pm 5$ & $\sqrt{ }$ & Friedman et al. (2010) & \\
\hline 147889 & 1.07 & 6.88 & 154 & $\sqrt{ }$ & Wszolk \& Godlowski (2003) & \\
\hline 147933 & 0.47 & 5.62 & 92 & $\times$ & Herbig (1975) & 58.2 \\
\hline 147933 & 0.46 & 5.62 & 54 & $\sqrt{ }$ & Herbig (1993) & \\
\hline 147933 & 0.45 & 5.62 & $50.8 \pm 2.4$ & $\sqrt{ }$ & Megier et al. (2005) & \\
\hline 147933 & 0.46 & 5.62 & 108 & $\times$ & Dorschner et al. (1977) & \\
\hline 147933 & 0.48 & 5.62 & $71 \pm 6$ & $\sqrt{ }$ & Friedman et al. (2010) & \\
\hline 147933 & 0.48 & 5.62 & 57 & $\sqrt{ }$ & Wszolk \& Godlowski (2003) & \\
\hline 149757 & 0.32 & 6.63 & 31 & $\sqrt{ }$ & Herbig (1993) & 31.2 \\
\hline 149757 & 0.29 & 6.63 & 30.5 & $\sqrt{ }$ & Galazutdinov et al. (2004) & \\
\hline 149757 & 0.29 & 6.63 & $30.5 \pm 1.5$ & $\sqrt{ }$ & Megier et al. (2005) & \\
\hline 149757 & 0.32 & 6.63 & $25.0 \pm 1.0$ & $\sqrt{ }$ & Sonnentrucher et al. (1997) & \\
\hline 149757 & 0.32 & 6.63 & $38 \pm 4$ & $\sqrt{ }$ & Friedman et al. (2010) & \\
\hline 149757 & 0.32 & 6.63 & 32 & $\sqrt{ }$ & Wszolk \& Godlowski (2003) & \\
\hline 152233 & 0.45 & 5.76 & 84.2 & $\sqrt{ }$ & Benvenuti \& Porceddu (1989) & 84.2 \\
\hline 152247 & 0.44 & 5.80 & 79.2 & $\sqrt{ }$ & Benvenuti \& Porceddu (1989) & 79.2 \\
\hline 152249 & 0.46 & 6.13 & 68.1 & $\sqrt{ }$ & Benvenuti \& Porceddu (1989) & 68.1 \\
\hline 154445 & 0.43 & 7.34 & $52.9 \pm 8.6$ & $\sqrt{ }$ & Désert et al. (1995) & 55.5 \\
\hline 154445 & 0.39 & 7.34 & $64.1 \pm 3.0$ & $\sqrt{ }$ & Megier et al. (2005) & \\
\hline 154445 & 0.42 & 7.34 & $42.0 \pm 1.3$ & $\sqrt{ }$ & Sonnentrucher et al. (1997) & \\
\hline 154445 & 0.42 & 7.34 & 63 & $\sqrt{ }$ & Wszolk \& Godlowski (2003) & \\
\hline 162978 & 0.35 & 5.32 & 59.9 & $\sqrt{ }$ & Benvenuti \& Porceddu (1989) & 59.0 \\
\hline 162978 & 0.35 & 5.32 & $58 \pm 9$ & $\sqrt{ }$ & Friedman et al. (2010) & \\
\hline 164794 & 0.33 & 6.31 & 34 & $\sqrt{ }$ & Benvenuti \& Porceddu (1989) & 34.0 \\
\hline 165052 & 0.44 & 5.68 & 55.9 & $\sqrt{ }$ & Benvenuti \& Porceddu (1989) & 55.9 \\
\hline 166937 & 0.24 & 6.23 & 133 & $\times$ & Herbig $(1975)$ & 72.7 \\
\hline 166937 & 0.26 & 6.23 & $69.7 \pm 1.0$ & $\sqrt{ }$ & Megier et al. (2005) & \\
\hline 166937 & 0.51 & 6.23 & $69.9 \pm 7.1$ & $\sqrt{ }$ & Désert et al. (1995) & \\
\hline 166937 & 0.25 & 6.23 & 80 & $\sqrt{ }$ & Benvenuti \& Porceddu (1989) & \\
\hline 166937 & 0.25 & 6.23 & $100 \pm 5$ & $\times$ & Friedman et al. (2010) & \\
\hline 166937 & 0.25 & 6.23 & 71 & $\sqrt{ }$ & Wszolk \& Godlowski (2003) & \\
\hline 167971 & 1.05 & 5.46 & $158.0 \pm 5.8$ & $\sqrt{ }$ & Megier et al. (2005) & 183.0 \\
\hline
\end{tabular}


Table 5-Continued

\begin{tabular}{|c|c|c|c|c|c|c|}
\hline $\mathrm{HD} / \mathrm{BD}$ & $\begin{array}{c}E(B-V) \\
\quad(\mathrm{mag})\end{array}$ & $\begin{array}{c}W_{2175} / E(B-V) \\
\left(\mu \mathrm{m}^{-1}\right)\end{array}$ & $\begin{array}{c}W_{5797} \\
(\mathrm{~m} \AA)\end{array}$ & $\begin{array}{c}\text { Adopt " } " \\
\text { or Reject "X" }\end{array}$ & Sources & $\begin{array}{c}W_{5797}(\mathrm{~m} \AA) \\
\text { This Work }\end{array}$ \\
\hline 167971 & 1.08 & 5.46 & 384 & $x$ & Herbig (1975) & \\
\hline 167971 & 1.08 & 5.46 & $208 \pm 6$ & $\sqrt{ }$ & Friedman et al. (2010) & \\
\hline 168076 & 0.80 & 4.81 & $167.2 \pm 12.8$ & $\times$ & Désert et al. (1995) & 265.6 \\
\hline 168076 & 0.81 & 4.81 & 281.1 & $\sqrt{ }$ & Benvenuti \& Porceddu (1989) & \\
\hline 168076 & 0.78 & 4.81 & $250 \pm 8$ & $\sqrt{ }$ & Friedman et al. (2010) & \\
\hline 168112 & 1.04 & 5.44 & $112.3 \pm 11.4$ & $\sqrt{ }$ & Désert et al. (1995) & 112.3 \\
\hline 183143 & 1.24 & 6.13 & $178.6 \pm 12.4$ & $\sqrt{ }$ & Désert et al. (1995) & 189.3 \\
\hline 183143 & 1.28 & 6.13 & 209.9 & $\sqrt{ }$ & Cox et al. (2007) & \\
\hline 183143 & 1.27 & 6.13 & $182 \pm 4$ & $\sqrt{ }$ & Snow et al. (2002) & \\
\hline 183143 & 1.28 & 6.13 & $189.4 \pm 10.2$ & $\sqrt{ }$ & Sonnentrucher et al. (1997) & \\
\hline 183143 & 1.28 & 6.13 & 237 & $x$ & Herbig (1993) & \\
\hline 183143 & 1.27 & 6.13 & $257 \pm 8$ & $\times$ & Friedman et al. (2010) & \\
\hline 183143 & 1.27 & 6.13 & $186.4 \pm 1.0$ & $\sqrt{ }$ & Hobbs et al. (2009) & \\
\hline 183143 & 1.27 & 6.13 & 234 & $\times$ & Wszolk \& Godlowski (2003) & \\
\hline 185418 & 0.50 & 6.67 & $105 \pm 5$ & $\sqrt{ }$ & Friedman et al. (2010) & 105.0 \\
\hline 190603 & 0.72 & 4.88 & $82.1 \pm 7.9$ & $\sqrt{ }$ & Sonnentrucher et al. (1997) & 79.5 \\
\hline 190603 & 0.71 & 4.88 & $86.7 \pm 3.2$ & $\sqrt{ }$ & Megier et al. (2005) & \\
\hline 190603 & 0.71 & 4.88 & 248 & $x$ & Herbig (1975) & \\
\hline 190603 & 0.94 & 4.88 & $69.6 \pm 2.8$ & $\sqrt{ }$ & Désert et al. (1995) & \\
\hline 192281 & 0.73 & 5.78 & $102.9 \pm 10.2$ & $\sqrt{ }$ & Désert et al. (1995) & 102.9 \\
\hline 193322 & 0.41 & 4.15 & 71 & $\sqrt{ }$ & Herbig (1993) & 67.5 \\
\hline 193322 & 0.41 & 4.15 & 64 & $\sqrt{ }$ & Wszolk \& Godlowski (2003) & \\
\hline 193682 & 0.83 & 7.40 & $73.0 \pm 5.8$ & $\sqrt{ }$ & Désert et al. (1995) & 73.0 \\
\hline 197770 & 0.58 & 6.82 & 85.8 & $\sqrt{ }$ & Cox et al. (2007) & 85.8 \\
\hline 198478 & 0.54 & 5.44 & 78.8 & $\sqrt{ }$ & Cox et al. (2007) & 77.8 \\
\hline 198478 & 0.54 & 5.44 & $72.0 \pm 4.0$ & $\sqrt{ }$ & Megier et al. (2005) & \\
\hline 198478 & 0.54 & 5.44 & $70.2 \pm 20.0$ & $\sqrt{ }$ & Sonnentrucher et al. (1997) & \\
\hline 198478 & 0.53 & 5.44 & 160 & $\times$ & Herbig (1975) & \\
\hline 198478 & 0.53 & 5.44 & 145 & $\times$ & Dorschner et al. (1977) & \\
\hline 198478 & 0.54 & 5.44 & $112 \pm 4$ & $x$ & Friedman et al. (2010) & \\
\hline 198478 & 0.54 & 5.44 & 90 & $\sqrt{ }$ & Wszolk \& Godlowski (2003) & \\
\hline 199216 & 0.73 & 5.89 & $97.1 \pm 2.2$ & $\sqrt{ }$ & Désert et al. (1995) & 97.1 \\
\hline 199478 & 0.47 & 6.42 & 235 & $\sqrt{ }$ & Herbig (1975) & 235.0 \\
\hline 199579 & 0.38 & 4.60 & $42.2 \pm 5.7$ & $\sqrt{ }$ & Désert et al. (1995) & 49.7 \\
\hline 199579 & 0.38 & 4.60 & 52 & $\sqrt{ }$ & Herbig (1993) & \\
\hline 199579 & 0.35 & 4.60 & $49.5 \pm 2.1$ & $\sqrt{ }$ & Megier et al. (2005) & \\
\hline 199579 & 0.36 & 4.60 & 105 & $\times$ & Herbig (1975) & \\
\hline 199579 & 0.37 & 4.60 & $50 \pm 4$ & $\sqrt{ }$ & Friedman et al. (2010) & \\
\hline 199579 & 0.37 & 4.60 & 55 & $\sqrt{ }$ & Wszolk \& Godlowski (2003) & \\
\hline 203938 & 0.66 & 5.70 & 117 & $\sqrt{ }$ & Galazutdinov et al. (2004) & 117.0 \\
\hline 203938 & 0.70 & 5.70 & $117.0 \pm 3.0$ & $\sqrt{ }$ & Megier et al. (2005) & \\
\hline 203938 & 0.74 & 5.70 & $152 \pm 5$ & $\times$ & Friedman et al. (2010) & \\
\hline 204827 & 1.06 & 4.25 & 168 & $x$ & Galazutdinov et al. (2004) & 199.0 \\
\hline 204827 & 1.11 & 4.25 & $199 \pm 3$ & $\sqrt{ }$ & Friedman et al. (2010) & \\
\hline 204827 & 1.11 & 4.25 & $199.0 \pm 1.1$ & $\sqrt{ }$ & Hobbs et al. (2008) & \\
\hline 206165 & 0.47 & 6.07 & $71.0 \pm 9.9$ & $\sqrt{ }$ & Sonnentrucher et al. (1997) & 80.7 \\
\hline 206165 & 0.48 & 6.07 & 87 & $\sqrt{ }$ & Herbig (1993) & \\
\hline 206165 & 0.46 & 6.07 & $76.6 \pm 1.1$ & $\sqrt{ }$ & Megier et al. (2005) & \\
\hline 206165 & 0.46 & 6.07 & 150 & $x$ & Dorschner et al. (1977) & \\
\hline 206165 & 0.47 & 6.07 & $106 \pm 5$ & $\times$ & Friedman et al. (2010) & \\
\hline 206165 & 0.47 & 6.07 & 88 & $\sqrt{ }$ & Wszolk \& Godlowski (2003) & \\
\hline 206267 & 0.50 & 4.96 & $89.8 \pm 1.6$ & $\sqrt{ }$ & Megier et al. (2005) & 96.7 \\
\hline 206267 & 0.49 & 4.96 & 90 & $\sqrt{ }$ & Galazutdinov et al. (2004) & \\
\hline 206267 & 0.53 & 4.96 & $102 \pm 5$ & $\sqrt{ }$ & Friedman et al. (2010) & \\
\hline 206267 & 0.53 & 4.96 & 105 & $\sqrt{ }$ & Wszolk \& Godlowski (2003) & \\
\hline 207198 & 0.62 & 5.23 & 139 & $\sqrt{ }$ & Herbig (1993) & 141.0 \\
\hline 207198 & 0.54 & 5.23 & $130.0 \pm 2.5$ & $\sqrt{ }$ & Megier et al. (2005) & \\
\hline 207198 & 0.56 & 5.23 & 140 & $\sqrt{ }$ & Galazutdinov et al. (2004) & \\
\hline 207198 & 0.62 & 5.23 & $144 \pm 3$ & $\sqrt{ }$ & Friedman et al. (2010) & \\
\hline 207198 & 0.62 & 5.23 & 152 & $\sqrt{ }$ & Wszolk \& Godlowski (2003) & \\
\hline 209339 & 0.37 & 6.74 & $62.2 \pm 1.9$ & $\sqrt{ }$ & Désert et al. (1995) & 62.2 \\
\hline 210121 & 0.40 & 2.74 & $46 \pm 9$ & $\sqrt{ }$ & Friedman et al. (2010) & 46.0 \\
\hline 216532 & 0.87 & 6.50 & $119.2 \pm 11.3$ & $\sqrt{ }$ & Désert et al. (1995) & 119.2 \\
\hline 216898 & 0.88 & 6.22 & $140.8 \pm 15.0$ & $\sqrt{ }$ & Désert et al. (1995) & 140.8 \\
\hline 217086 & 0.92 & 6.48 & $144.4 \pm 8.3$ & $\sqrt{ }$ & Désert et al. (1995) & 149.2 \\
\hline 217086 & 0.92 & 6.48 & 154 & $\sqrt{ }$ & Galazutdinov et al. (2004) & \\
\hline
\end{tabular}


Table 5-Continued

\begin{tabular}{|c|c|c|c|c|c|c|}
\hline $\mathrm{HD} / \mathrm{BD}$ & $\begin{array}{c}E(B-V) \\
\quad(\mathrm{mag})\end{array}$ & $\begin{array}{c}W_{2175} / E(B-V) \\
\left(\mu \mathrm{m}^{-1}\right)\end{array}$ & $\begin{array}{c}W_{5797} \\
(\mathrm{~m} \AA)\end{array}$ & $\begin{array}{l}\text { Adopt " } \sqrt{ } " \\
\text { or Reject "X" }\end{array}$ & Sources & $\begin{array}{c}W_{5797}(\mathrm{~m} \AA) \\
\text { This Work }\end{array}$ \\
\hline 239729 & 0.67 & 4.68 & $73.7 \pm 2.0$ & $\sqrt{ }$ & Désert et al. (1995) & 73.7 \\
\hline 242908 & 0.62 & 5.13 & $47.7 \pm 3.7$ & $\sqrt{ }$ & Désert et al. (1995) & 47.7 \\
\hline 303308 & 0.46 & 4.86 & 57 & $\sqrt{ }$ & Benvenuti \& Porceddu (1989) & 57.0 \\
\hline+631964 & 0.95 & 5.68 & $224.2 \pm 5.7$ & $\sqrt{ }$ & Désert et al. (1995) & 223.8 \\
\hline+631964 & 0.96 & 5.68 & $223.4 \pm 3.0$ & $\sqrt{ }$ & Megier et al. (2005) & \\
\hline+631964 & 1.00 & 5.68 & $295 \pm 9$ & $\times$ & Friedman et al. (2010) & \\
\hline
\end{tabular}


Table 6. Same as Table 3 but for the $\lambda 6195 \AA / 6196 \AA$ DIB.

\begin{tabular}{|c|c|c|c|c|c|c|c|c|}
\hline $\mathrm{HD} / \mathrm{BD}$ & $\begin{array}{c}E(B-V) \\
\quad(\mathrm{mag})\end{array}$ & $\begin{array}{c}W_{2175} / E(B-V) \\
\left(\mu \mathrm{m}^{-1}\right)\end{array}$ & $\begin{array}{c}W_{6195} \\
(\mathrm{~m} \AA)\end{array}$ & $\begin{array}{c}\text { Adopt "V" } \\
\text { or Reject "X" }\end{array}$ & $\begin{array}{c}W_{6196} \\
(\mathrm{~m} \AA)\end{array}$ & $\begin{array}{c}\text { Adopt "V" } \\
\text { or Reject "×" }\end{array}$ & Sources & $\begin{array}{c}W_{6195 / 6196}(\mathrm{~m} \AA) \\
\text { This Work }\end{array}$ \\
\hline 2905 & 0.33 & 4.05 & - & $x$ & $30.9 \pm 1.6$ & $\sqrt{ }$ & McCall et al. (2010) & 32.8 \\
\hline 2905 & 0.32 & 4.05 & 59 & $x$ & - & $x$ & Herbig (1975) & \\
\hline 2905 & 0.33 & 4.05 & - & $x$ & $32.6 \pm 1.3$ & $\checkmark$ & Megier et al. (2005) & \\
\hline 2905 & 0.33 & 4.05 & - & $x$ & $35 \pm 2$ & $\sqrt{ }$ & Friedman et al. (2010) & \\
\hline 15558 & 0.75 & 5.78 & $44.3 \pm 3.0$ & $\hat{\jmath}$ & - & 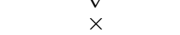 & Désert et al. (1995) & 44.3 \\
\hline 21291 & 0.41 & 7.31 & 42 & $x$ & - & $x$ & Herbig (1975) & 23.5 \\
\hline 21291 & 0.43 & 7.31 & - & $\hat{x}$ & 20.2 & $\sqrt{ }$ & Cox et al. (2007) & \\
\hline 21291 & 0.42 & 7.31 & - & $\hat{x}$ & $26.8 \pm 1.2$ & $\sqrt{ }$ & Megier et al. (2005) & \\
\hline 21483 & 0.56 & 4.07 & - & $\hat{x}$ & $23.2 \pm 1.6$ & $\sqrt{ }$ & McCall et al. (2010) & 22.9 \\
\hline 21483 & 0.56 & 4.07 & - & $x$ & $22.5 \pm 1.5$ & $\checkmark$ & Friedman et al. (2010) & \\
\hline 27778 & 0.37 & 4.75 & - & $\hat{x}$ & $12.9 \pm 1.0$ & $\sqrt{ }$ & McCall et al. (2010) & 12.2 \\
\hline 27778 & 0.37 & 4.75 & - & $\hat{x}$ & $11.5 \pm 0.5$ & $\sqrt{ }$ & Friedman et al. (2010) & \\
\hline 29647 & 1.00 & 3.82 & - & $\hat{x}$ & $12.1 \pm 1.6$ & $\sqrt{ }$ & McCall et al. (2010) & 10.9 \\
\hline 29647 & 1.00 & 3.82 & - & $x$ & $9.7 \pm 1.4$ & $\sqrt{ }$ & Friedman et al. (2010) & \\
\hline 30614 & 0.30 & 5.36 & - & $\hat{x}$ & $16.1 \pm 1.0$ & $\sqrt{ }$ & McCall et al. (2010) & 16.5 \\
\hline 30614 & 0.32 & 5.36 & $16.0 \pm 2.6$ & $\hat{\mathfrak{v}}$ & ${ }_{-}^{10.0}$ & 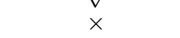 & Désert et al. (1995) & \\
\hline 30614 & 0.26 & 5.36 & - & $x$ & $16.6 \pm 0.4$ & $\hat{v}$ & Megier et al. (2005) & \\
\hline 30614 & 0.30 & 5.36 & _- & $\hat{x}$ & $17.2 \pm 1.5$ & $\sqrt{ }$ & Friedman et al. (2010) & \\
\hline+31643 & 0.85 & 6.59 & $34.9 \pm 11.1$ & $\hat{\jmath}$ & - & $\times$ & Désert et al. (1995) & 34.9 \\
\hline 34078 & 0.52 & 6.00 & + & 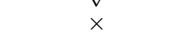 & $25.4 \pm 2.2$ & $\hat{\sqrt{ }}$ & McCall et al. (2010) & 23.1 \\
\hline 34078 & 0.52 & 6.00 & 57 & $\hat{x}$ & - & $x$ & Herbig (1975) & \\
\hline 34078 & 0.49 & 6.00 & - & $x$ & $15.9 \pm 1.1$ & $x$ & Megier et al. (2005) & \\
\hline 34078 & 0.52 & 6.00 & $20.8 \pm 2.6$ & $\hat{v}$ & - & $x$ & Désert et al. (1995) & \\
\hline 34078 & 0.52 & 6.00 & - & $x$ & $23 \pm 1$ & $\sqrt{ }$ & Friedman et al. (2010) & \\
\hline 36879 & 0.50 & 4.92 & $29.0 \pm 3.5$ & $\hat{\jmath}$ & - & $x$ & Désert et al. (1995) & 29.0 \\
\hline 37022 & 0.34 & 2.47 & - & 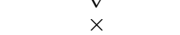 & $6.4 \pm 1.0$ & $\hat{\sqrt{ }}$ & McCall et al. (2010) & 5.5 \\
\hline 37022 & 0.34 & 2.47 & - & $\hat{x}$ & $4.5 \pm 0.7$ & $\sqrt[v]{ }$ & Friedman et al. (2010) & \\
\hline 37061 & 0.52 & 3.42 & - & $\hat{x}$ & $12.6 \pm 1.5$ & $\sqrt{ }$ & McCall et al. (2010) & 12.6 \\
\hline 37061 & 0.50 & 3.42 & - & $\hat{x}$ & $20.0 \pm 1.7$ & $x$ & Megier et al. (2005) & \\
\hline 37061 & 0.52 & 3.42 & - & $x$ & $12.6 \pm 1.5$ & $\sqrt{ }$ & Friedman et al. (2010) & \\
\hline 37367 & 0.43 & 7.15 & $45.2 \pm 6.5$ & $\hat{v}$ & - & $x$ & Désert et al. (1995) & 42.2 \\
\hline 37367 & 0.40 & 7.15 & - & $x$ & $41.5 \pm 1.9$ & $\hat{\sqrt{ }}$ & McCall et al. (2010) & \\
\hline 37367 & 0.40 & 7.15 & - & $\hat{x}$ & $40 \pm 1$ & $\sqrt{v}$ & Friedman et al. (2010) & \\
\hline 37903 & 0.38 & 3.64 & $16.0 \pm 2.7$ & $\hat{\jmath}$ & - & $\times$ & Désert et al. (1995) & 13.5 \\
\hline 37903 & 0.35 & 3.64 & - & $x$ & $13.1 \pm 11.8$ & $\hat{v}$ & McCall et al. (2010) & \\
\hline 37903 & 0.35 & 3.64 & _- & $\hat{x}$ & $11.5 \pm 1.5$ & $\sqrt{v}$ & Friedman et al. (2010) & \\
\hline 38087 & 0.29 & 6.03 & - & $\hat{x}$ & $12.2 \pm 1.1$ & $\sqrt{ }$ & McCall et al. (2010) & 12.4 \\
\hline 38087 & 0.29 & 6.03 & - & $x$ & $12.6 \pm 1$ & $\checkmark$ & Friedman et al. (2010) & \\
\hline 38131 & 0.51 & 6.61 & $45.9 \pm 5.6$ & $\hat{\jmath}$ & - & $x$ & Désert et al. (1995) & 45.9 \\
\hline 40893 & 0.46 & 5.92 & - & $\begin{array}{l}\mathrm{v} \\
\mathrm{x}\end{array}$ & $39 \pm 1.5$ & $\hat{\sqrt{ }}$ & Friedman et al. (2010) & 39.0 \\
\hline 41117 & 0.45 & 5.89 & - & $\hat{x}$ & $41.3 \pm 0.9$ & $\sqrt{ }$ & McCall et al. (2010) & 41.6 \\
\hline 41117 & 0.44 & 5.89 & - & $\hat{x}$ & $41.5 \pm 0.8$ & $\sqrt{ }$ & Megier et al. (2005) & \\
\hline 41117 & 0.45 & 5.89 & - & $\hat{x}$ & $\begin{array}{l}42 \pm 1 \\
42.0\end{array}$ & $\sqrt[v]{ }$ & Friedman et al. (2010) & \\
\hline 42087 & 0.35 & 6.52 & - & $\hat{x}$ & $31.1 \pm 1.8$ & $\sqrt{ }$ & Megier et al. (2005) & 30.8 \\
\hline 42087 & 0.36 & 6.52 & - & $\hat{x}$ & $31.2 \pm 1.5$ & $\sqrt{ }$ & McCall et al. (2010) & \\
\hline 42087 & 0.36 & 6.52 & - & $x$ & $30 \pm 1$ & $\checkmark$ & Friedman et al. (2010) & \\
\hline 46056 & 0.50 & 5.11 & - & $\hat{x}$ & $34.7 \pm 12.1$ & $\sqrt{ }$ & McCall et al. (2010) & 33.4 \\
\hline 46056 & 0.50 & 5.11 & - & $\hat{x}$ & $32 \pm 1.5$ & $\sqrt{ }$ & Friedman et al. (2010) & \\
\hline 46202 & 0.49 & 4.74 & - & $\hat{x}$ & $36.5 \pm 2.0$ & $\sqrt{ }$ & McCall et al. (2010) & 35.8 \\
\hline 46202 & 0.49 & 4.74 & _- & $\hat{x}$ & $35 \pm 3$ & $\sqrt{ }$ & Friedman et al. (2010) & \\
\hline 47129 & 0.36 & 6.38 & - & $\hat{x}$ & $22.8 \pm 1.3$ & $\sqrt{v}$ & McCall et al. (2010) & 21.3 \\
\hline 47129 & 0.34 & 6.38 & _- & $\hat{x}$ & $17.2 \pm 0.7$ & $\sqrt{ }$ & Megier et al. (2005) & \\
\hline 47129 & 0.36 & 6.38 & - & $\hat{x}$ & $24 \pm 1.5$ & $\sqrt{ }$ & Friedman et al. (2010) & \\
\hline 48099 & 0.27 & 5.63 & - & $x$ & $20.6 \pm 1.4$ & $\sqrt{ }$ & McCall et al. (2010) & 19.9 \\
\hline 48099 & 0.27 & 5.63 & - & $\hat{x}$ & $19.2 \pm 0.8$ & $\sqrt{ }$ & Friedman et al. (2010) & \\
\hline 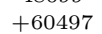 & 0.89 & 5.94 & $57.9 \pm 5.3$ & $\hat{\jmath}$ & - & $\times$ & Désert et al. (1995) & 57.9 \\
\hline 122879 & 0.35 & 6.83 & - & $x$ & 24.8 & $\sqrt{ }$ & Benvenuti \& Porceddu (1989) & 25.3 \\
\hline 122879 & 0.34 & 6.83 & - & $\hat{x}$ & $25.7 \pm 1.2$ & $\sqrt{ }$ & Megier et al. (2005) & 47.3 \\
\hline 123008 & 0.63 & 4.94 & - & $\hat{x}$ & 47.3 & $\sqrt{ }$ & Benvenuti \& Porceddu (1989) & \\
\hline 142096 & 0.19 & 4.46 & _- & $\hat{x}$ & $6.6 \pm 0.3$ & $\sqrt{ }$ & Megier et al. (2005) & 6.6 \\
\hline 143018 & 0.05 & 5.12 & - & $\hat{x}$ & $2.5 \pm 0.8$ & $\sqrt{ }$ & Friedman et al. (2010) & 2.5 \\
\hline 143275 & 0.17 & 5.27 & - & $x$ & 7 & $\checkmark$ & Wszolk \& Godlowski (2003) & 7.4 \\
\hline 143275 & 0.17 & 5.27 & - & $\hat{x}$ & $7.8 \pm 0.8$ & $\sqrt{ }$ & Friedman et al. (2010) & \\
\hline 144217 & 0.19 & 6.49 & - & $\hat{x}$ & $14.6 \pm 1.6$ & $\sqrt{ }$ & McCall et al. (2010) & 13.6 \\
\hline 144217 & 0.19 & 6.50 & - & $\mathrm{x}$ & 13 & $\sqrt{ }$ & Wszolk \& Godlowski (2003) & \\
\hline 144217 & 0.19 & 6.50 & - & $x$ & $13.2 \pm 0.8$ & $\sqrt{ }$ & Friedman et al. (2010) & \\
\hline
\end{tabular}


Table 6-Continued

\begin{tabular}{|c|c|c|c|c|c|c|c|c|}
\hline $\mathrm{HD} / \mathrm{BD}$ & $\begin{array}{c}E(B-V) \\
\quad(\mathrm{mag})\end{array}$ & $\begin{array}{c}W_{2175} / E(B-V) \\
\left(\mu \mathrm{m}^{-1}\right)\end{array}$ & $\begin{array}{c}W_{6195} \\
(\mathrm{~m} \AA)\end{array}$ & $\begin{array}{c}\text { Adopt " } \sqrt{ } \text { " } \\
\text { or Reject "X" }\end{array}$ & $\begin{array}{c}W_{6196} \\
(\mathrm{~m} \AA)\end{array}$ & $\begin{array}{c}\text { Adopt "V" } \\
\text { or Reject "X" }\end{array}$ & Sources & $\begin{array}{c}W_{6195 / 6196}(\mathrm{~m} \AA) \\
\text { This Work }\end{array}$ \\
\hline 144470 & 0.22 & 6.62 & - & $x$ & $17.7 \pm 1.6$ & $\checkmark$ & McCall et al. (2010) & 16.8 \\
\hline 144470 & 0.19 & 6.62 & - & $x$ & $15.2 \pm 0.4$ & $\checkmark$ & Megier et al. (2005) & \\
\hline 144470 & 0.19 & 6.62 & - & $x$ & 18 & $\sqrt{ }$ & Benvenuti \& Porceddu (1989) & \\
\hline 144470 & 0.22 & 6.62 & - & $\hat{x}$ & 16 & $\sqrt{v}$ & Wszolk \& Godlowski (2003) & \\
\hline 144470 & 0.22 & 6.62 & - & $x$ & $17 \pm 1$ & $\sqrt{ }$ & Friedman et al. (2010) & \\
\hline 145502 & 0.24 & 6.25 & - & $x$ & $17.0 \pm 1.3$ & $\checkmark$ & McCall et al. (2010) & 15.7 \\
\hline 145502 & 0.24 & 6.25 & - & $x$ & 14 & $\checkmark$ & Wszolk \& Godlowski (2003) & \\
\hline 145502 & 0.24 & 6.25 & - & $\hat{x}$ & $16 \pm 1$ & $\checkmark$ & Friedman et al. (2010) & \\
\hline 147165 & 0.41 & 5.27 & - & $x$ & $21.6 \pm 1.7$ & $\sqrt{ }$ & McCall et al. (2010) & 17.8 \\
\hline 147165 & 0.39 & 5.27 & 32 & $\hat{x}$ & - & $\times$ & Herbig (1975) & \\
\hline 147165 & 0.34 & 5.27 & - & $x$ & $16.5 \pm 0.5$ & $\sqrt{ }$ & Megier et al. (2005) & \\
\hline 147165 & 0.41 & 5.27 & - & $x$ & 18 & $\sqrt{ }$ & Wszolk \& Godlowski (2003) & \\
\hline 147165 & 0.41 & 5.27 & - & $x$ & $15.2 \pm 0.7$ & $\checkmark$ & Friedman et al. (2010) & \\
\hline 147888 & 0.47 & 5.87 & - & $x$ & $20.2 \pm 1.1$ & $\sqrt{ }$ & McCall et al. (2010) & 19.6 \\
\hline 147888 & 0.47 & 5.87 & - & $x$ & $19 \pm 1$ & $\sqrt{ }$ & Friedman et al. (2010) & \\
\hline 147889 & 1.08 & 6.88 & 79 & $\hat{x}$ & - & $x$ & Herbig (1975) & 45.8 \\
\hline 147889 & 1.07 & 6.88 & - & $x$ & $45.5 \pm 1.7$ & $\checkmark$ & McCall et al. (2010) & \\
\hline 147889 & 1.07 & 6.88 & - & $\hat{x}$ & $46 \pm 2$ & $\checkmark$ & Friedman et al. (2010) & \\
\hline 147933 & 0.47 & 5.62 & 31 & $x$ & - & $\times$ & Herbig (1975) & 16.9 \\
\hline 147933 & 0.48 & 5.62 & - & $x$ & $17.1 \pm 1.6$ & $\sqrt{ }$ & McCall et al. (2010) & \\
\hline 147933 & 0.45 & 5.62 & - & $x$ & $16.6 \pm 0.7$ & $\sqrt{ }$ & Megier et al. (2005) & \\
\hline 147933 & 0.48 & 5.62 & - & $x$ & $17.1 \pm 1$ & $\sqrt{ }$ & Friedman et al. (2010) & \\
\hline 149757 & 0.32 & 6.63 & - & $x$ & $11.5 \pm 1.2$ & $\sqrt{ }$ & McCall et al. (2010) & 10.9 \\
\hline 149757 & 0.29 & 6.63 & - & $\hat{x}$ & $11.0 \pm 0.5$ & $\checkmark$ & Megier et al. (2005) & \\
\hline 149757 & 0.32 & 6.63 & - & $x$ & 11 & $\sqrt{ }$ & Wszolk \& Godlowski (2003) & \\
\hline 149757 & 0.32 & 6.63 & - & $\hat{x}$ & $10 \pm 1$ & $\sqrt{ }$ & Friedman et al. (2010) & \\
\hline 152233 & 0.45 & 5.76 & - & $\hat{x}$ & 23.9 & $\sqrt{ }$ & Benvenuti \& Porceddu (1989) & 23.9 \\
\hline 152247 & 0.44 & 5.80 & - & $x$ & 21.1 & $\sqrt{ }$ & Benvenuti \& Porceddu (1989) & 21.1 \\
\hline 152248 & 0.45 & 5.24 & - & $x$ & 22.1 & $\sqrt{ }$ & Benvenuti \& Porceddu (1989) & 22.1 \\
\hline 152249 & 0.46 & 6.13 & - & $\hat{x}$ & 22.1 & $\checkmark$ & Benvenuti \& Porceddu (1989) & 22.1 \\
\hline 154445 & 0.43 & 7.34 & $17.2 \pm 3.0$ & $\checkmark$ & - & $x$ & Désert et al. (1995) & 20.7 \\
\hline 154445 & 0.39 & 7.34 & - & $\times$ & $24.1 \pm 1.4$ & $\sqrt{ }$ & Megier et al. (2005) & \\
\hline 162978 & 0.35 & 5.32 & - & $x$ & $18.6 \pm 1.7$ & $\sqrt{ }$ & McCall et al. (2010) & 20.4 \\
\hline 162978 & 0.35 & 5.32 & - & $x$ & 21 & $\sqrt{ }$ & Benvenuti \& Porceddu (1989) & \\
\hline 162978 & 0.35 & 5.32 & - & $\hat{x}$ & $21.5 \pm 2$ & $\sqrt{ }$ & Friedman et al. (2010) & \\
\hline 164794 & 0.33 & 6.31 & - & $\hat{x}$ & 17.2 & $\sqrt{ }$ & Benvenuti \& Porceddu (1989) & 17.2 \\
\hline 165052 & 0.44 & 5.68 & - & $x$ & 19.8 & $\checkmark$ & Benvenuti \& Porceddu (1989) & 19.8 \\
\hline 166937 & 0.25 & 6.23 & - & $\hat{x}$ & $25.9 \pm 1.2$ & $\sqrt{ }$ & McCall et al. (2010) & 24.5 \\
\hline 166937 & 0.24 & 6.23 & 47 & $\hat{x}$ & - & $\times$ & Herbig (1975) & \\
\hline 166937 & 0.51 & 6.23 & $21.9 \pm 2.0$ & $\hat{v}$ & - & $x$ & Désert et al. (1995) & \\
\hline 166937 & 0.26 & 6.23 & - & $x$ & $23.2 \pm 0.5$ & $\sqrt{ }$ & Megier et al. (2005) & \\
\hline 166937 & 0.25 & 6.23 & - & $x$ & 26 & $\checkmark$ & Benvenuti \& Porceddu (1989) & \\
\hline 166937 & 0.25 & 6.23 & - & $x$ & $25.7 \pm 1$ & $\sqrt{ }$ & Friedman et al. (2010) & \\
\hline 167971 & 1.08 & 5.46 & 94 & $\hat{x}$ & - & $x$ & Herbig (1975) & 58.5 \\
\hline 167971 & 1.08 & 5.46 & - & $x$ & $55.9 \pm 1.1$ & $\sqrt{ }$ & McCall et al. (2010) & \\
\hline 167971 & 1.05 & 5.46 & - & $x$ & $61.5 \pm 4.3$ & $\checkmark$ & Megier et al. (2005) & \\
\hline 167971 & 1.08 & 5.46 & - & $\hat{x}$ & $58 \pm 2$ & $\sqrt{ }$ & Friedman et al. (2010) & \\
\hline 168076 & 0.78 & 4.81 & - & $\hat{x}$ & $61.4 \pm 1.7$ & $\checkmark$ & McCall et al. (2010) & 60.5 \\
\hline 168076 & 0.80 & 4.81 & $39.2 \pm 3.2$ & $x$ & - & $x$ & Désert et al. (1995) & \\
\hline 168076 & 0.81 & 4.81 & - & $\hat{x}$ & 46.2 & $x$ & Benvenuti \& Porceddu (1989) & \\
\hline 168076 & 0.78 & 4.81 & - & $x$ & $59.5 \pm 3$ & $\sqrt{ }$ & Friedman et al. (2010) & \\
\hline 168112 & 1.04 & 5.44 & $44.7 \pm 6.2$ & $\hat{v}$ & - & $x$ & Désert et al. (1995) & 44.7 \\
\hline 183143 & 1.24 & 6.13 & $95.5 \pm 1.2$ & $\sqrt{ }$ & - & $\hat{x}$ & Désert et al. (1995) & 90.3 \\
\hline 183143 & 1.27 & 6.13 & - & $\times$ & $90.2 \pm 1.2$ & $\hat{v}$ & McCall et al. (2010) & \\
\hline 183143 & 1.28 & 6.13 & - & $\hat{x}$ & 81.9 & $\sqrt{ }$ & Cox et al. (2007) & \\
\hline 183143 & 1.27 & 6.13 & - & $x$ & $90.4 \pm 0.7$ & $\sqrt{ }$ & Hobbs et al. (2009) & \\
\hline 183143 & 1.27 & 6.13 & - & $x$ & 95 & $\checkmark$ & Wszolk \& Godlowski (2003) & \\
\hline 183143 & 1.27 & 6.13 & - & $x$ & $89 \pm 2$ & $\checkmark$ & Friedman et al. (2010) & \\
\hline 185418 & 0.50 & 6.67 & - & $\hat{x}$ & $35.3 \pm 1.0$ & $\sqrt{ }$ & McCall et al. (2010) & 35.2 \\
\hline 185418 & 0.50 & 6.67 & - & $\hat{x}$ & $35 \pm 1$ & $\checkmark$ & Friedman et al. (2010) & \\
\hline 190603 & 0.71 & 4.88 & 77 & $\hat{x}$ & - & $\times$ & Herbig (1975) & 32.5 \\
\hline 190603 & 0.71 & 4.88 & - & $x$ & $33.0 \pm 1.6$ & $\sqrt{ }$ & Megier et al. (2005) & \\
\hline 190603 & 0.94 & 4.88 & $32.0 \pm 3.8$ & $\hat{v}$ & - & $x$ & Désert et al. (1995) & \\
\hline 192281 & 0.73 & 5.78 & $32.9 \pm 2.9$ & $\sqrt{ }$ & - & $x$ & Désert et al. (1995) & 32.9 \\
\hline 193682 & 0.83 & 7.40 & $58.9 \pm 4.2$ & $\checkmark$ & - & $x$ & Désert et al. (1995) & 58.9 \\
\hline 197770 & 0.58 & 6.82 & - & $x$ & 24.9 & $\hat{v}$ & Cox et al. (2007) & 24.9 \\
\hline
\end{tabular}


Table 6-Continued

\begin{tabular}{|c|c|c|c|c|c|c|c|c|}
\hline $\mathrm{HD} / \mathrm{BD}$ & $\begin{array}{c}E(B-V) \\
\quad(\mathrm{mag})\end{array}$ & $\begin{array}{c}W_{2175} / E(B-V) \\
\left(\mu \mathrm{m}^{-1}\right)\end{array}$ & $\begin{array}{c}W_{6195} \\
(\mathrm{~m} \AA)\end{array}$ & $\begin{array}{l}\text { Adopt "V" } \\
\text { or Reject "X" }\end{array}$ & $\begin{array}{c}W_{6196} \\
(\mathrm{~m} \AA)\end{array}$ & $\begin{array}{c}\text { Adopt "V" } \\
\text { or Reject "X" }\end{array}$ & Sources & $\begin{array}{c}W_{6195 / 6196}(\mathrm{~m} \AA) \\
\text { This Work }\end{array}$ \\
\hline 198478 & 0.54 & 5.44 & - & $x$ & $34.9 \pm 1.4$ & $\checkmark$ & McCall et al. (2010) & 33.9 \\
\hline 198478 & 0.53 & 5.44 & 40 & $x$ & - & $x$ & Herbig (1975) & \\
\hline 198478 & 0.54 & 5.44 & - & $x$ & 34.0 & $\sqrt{ }$ & Cox et al. (2007) & \\
\hline 198478 & 0.54 & 5.44 & - & $x$ & $33.6 \pm 0.7$ & $\checkmark$ & Megier et al. (2005) & \\
\hline 198478 & 0.54 & 5.44 & - & $x$ & $33.1 \pm 1.5$ & $\sqrt{ }$ & Friedman et al. (2010) & \\
\hline 199216 & 0.73 & 5.89 & $23.4 \pm 2.9$ & $\checkmark$ & - & $x$ & Désert et al. (1995) & 23.4 \\
\hline 199478 & 0.47 & 6.42 & 35 & $\sqrt{ }$ & - & $x$ & Herbig (1975) & 35.0 \\
\hline 199579 & 0.38 & 4.60 & $12.2 \pm 2.0$ & $\checkmark$ & - & $x$ & Désert et al. (1995) & 14.7 \\
\hline 199579 & 0.37 & 4.60 & - & $x$ & $15.8 \pm 1.0$ & $\sqrt{ }$ & McCall et al. (2010) & \\
\hline 199579 & 0.35 & 4.60 & - & $x$ & $15.3 \pm 1.0$ & $\checkmark$ & Megier et al. (2005) & \\
\hline 199579 & 0.37 & 4.60 & - & $x$ & $15.5 \pm 1$ & $\sqrt{ }$ & Friedman et al. (2010) & \\
\hline 203938 & 0.70 & 5.70 & - & $x$ & $36.1 \pm 1.6$ & $\checkmark$ & Megier et al. (2005) & 40.5 \\
\hline 203938 & 0.74 & 5.70 & - & $x$ & $43.3 \pm 1.1$ & $\sqrt{ }$ & McCall et al. (2010) & \\
\hline 203938 & 0.74 & 5.70 & - & $x$ & $42 \pm 1$ & $\checkmark$ & Friedman et al. (2010) & \\
\hline 204827 & 1.11 & 4.25 & - & $x$ & $41.9 \pm 11.5$ & $\sqrt{ }$ & McCall et al. (2010) & 40.4 \\
\hline 204827 & 1.11 & 4.25 & - & $x$ & $37.8 \pm 0.6$ & $\checkmark$ & Hobbs et al. (2008) & \\
\hline 204827 & 1.11 & 4.25 & - & $x$ & $41.5 \pm 1$ & $\sqrt{ }$ & Friedman et al. (2010) & \\
\hline 206165 & 0.47 & 6.07 & - & $x$ & $27.4 \pm 1.6$ & $\sqrt{ }$ & McCall et al. (2010) & 26.2 \\
\hline 206165 & 0.46 & 6.07 & - & $x$ & $24.2 \pm 0.6$ & $\sqrt{ }$ & Megier et al. (2005) & \\
\hline 206165 & 0.47 & 6.07 & - & $x$ & 27 & $\checkmark$ & Wszolk \& Godlowski (2003) & \\
\hline 206165 & 0.47 & 6.07 & - & $x$ & $26 \pm 1$ & $\sqrt{ }$ & Friedman et al. (2010) & \\
\hline 206267 & 0.53 & 4.96 & - & $x$ & $30.2 \pm 1.1$ & $\checkmark$ & McCall et al. (2010) & 28.9 \\
\hline 206267 & 0.50 & 4.96 & - & $x$ & $27.3 \pm 0.5$ & $\checkmark$ & Megier et al. (2005) & \\
\hline 206267 & 0.53 & 4.96 & - & $x$ & 29 & $\checkmark$ & Wszolk \& Godlowski (2003) & \\
\hline 206267 & 0.53 & 4.96 & - & $x$ & $29 \pm 1$ & $\sqrt{ }$ & Friedman et al. (2010) & \\
\hline 207198 & 0.54 & 5.23 & - & $x$ & $34.5 \pm 1.2$ & $\checkmark$ & Megier et al. (2005) & 32.3 \\
\hline 207198 & 0.62 & 5.23 & - & $x$ & $31.7 \pm 1.0$ & $\sqrt{ }$ & McCall et al. (2010) & \\
\hline 207198 & 0.62 & 5.23 & - & $x$ & $30 \pm 1$ & $\sqrt{ }$ & Friedman et al. (2010) & \\
\hline 207198 & 0.62 & 5.23 & - & $x$ & 33 & $\checkmark$ & Wszolk \& Godlowski (2003) & \\
\hline 209339 & 0.37 & 6.74 & $25.2 \pm 0.7$ & $\checkmark$ & - & $x$ & Désert et al. (1995) & 25.2 \\
\hline 210121 & 0.40 & 2.74 & - & $x$ & $11.2 \pm 11.3$ & $\checkmark$ & McCall et al. (2010) & 10.3 \\
\hline 210121 & 0.40 & 2.74 & - & $x$ & $9.4 \pm 0.7$ & $\checkmark$ & Friedman et al. (2010) & \\
\hline 216532 & 0.87 & 6.50 & $39.2 \pm 3.5$ & $\checkmark$ & - & $x$ & Désert et al. (1995) & 39.2 \\
\hline 216898 & 0.88 & 6.22 & $59.8 \pm 6.2$ & $\checkmark$ & - & $x$ & Désert et al. (1995) & 59.8 \\
\hline 217086 & 0.92 & 6.48 & $48.8 \pm 3.7$ & $\sqrt{ }$ & - & $x$ & Désert et al. (1995) & 48.8 \\
\hline 239729 & 0.67 & 4.68 & $32.2 \pm 1.3$ & $\checkmark$ & - & $x$ & Désert et al. (1995) & 32.2 \\
\hline 303308 & 0.46 & 4.86 & - & $x$ & 22.1 & $\checkmark$ & Benvenuti \& Porceddu (1989) & 22.1 \\
\hline+631964 & 1.00 & 5.68 & - & $x$ & $89.6 \pm 2.2$ & $\checkmark$ & McCall et al. (2010) & 86.8 \\
\hline+631964 & 0.95 & 5.68 & $57.0 \pm 5.7$ & $x$ & - & $x$ & Désert et al. (1995) & \\
\hline+631964 & 0.96 & 5.68 & - & $x$ & $78.8 \pm 3.2$ & $\checkmark$ & Megier et al. (2005) & \\
\hline+631964 & 1.00 & 5.68 & - & $x$ & $92 \pm 2$ & $\checkmark$ & Friedman et al. (2010) & \\
\hline
\end{tabular}


Table 7. Same as Table 3 but for the $\lambda 6203 \AA$ DIB.

\begin{tabular}{|c|c|c|c|c|c|c|}
\hline $\mathrm{HD} / \mathrm{BD}$ & $\begin{array}{c}E(B-V) \\
\quad(\mathrm{mag})\end{array}$ & $\begin{array}{c}W_{2175} / E(B-V) \\
\left(\mu \mathrm{m}^{-1}\right)\end{array}$ & $\begin{array}{l}W_{6203} \\
(\mathrm{~m} \AA)\end{array}$ & $\begin{array}{l}\text { Adopt " } \sqrt{ } \text { " } \\
\text { or Reject "X" }\end{array}$ & Sources & $\begin{array}{c}W_{6203}(\mathrm{~m} \AA) \\
\text { This Work }\end{array}$ \\
\hline 2905 & 0.32 & 4.05 & 81 & $\sqrt{ }$ & Herbig (1975) & 81.0 \\
\hline 21291 & 0.42 & 7.31 & $48.1 \pm 3.1$ & $\times$ & Megier et al. (2005) & 177.0 \\
\hline 21291 & 0.41 & 7.31 & 177 & $\sqrt{ }$ & Herbig (1975) & \\
\hline 21483 & 0.56 & 4.07 & $71 \pm 5$ & $\sqrt{ }$ & Thorburn et al. (2003) & 71.0 \\
\hline 27778 & 0.37 & 4.75 & $34 \pm 3$ & $\sqrt{ }$ & Thorburn et al. (2003) & 34.0 \\
\hline 30614 & 0.30 & 5.36 & $63.7 \pm 6$ & $\sqrt{ }$ & Thorburn et al. (2003) & \\
\hline 34078 & 0.52 & 6.00 & 94 & $\sqrt{ }$ & Herbig (1975) & 102.5 \\
\hline 34078 & 0.49 & 6.00 & $27.9 \pm 2.7$ & $\times$ & Megier et al. (2005) & \\
\hline 34078 & 0.52 & 6.00 & $111 \pm 4$ & $\sqrt{ }$ & Thorburn et al. (2003) & \\
\hline 37061 & 0.50 & 3.42 & $26.4 \pm 2.4$ & $\times$ & Megier et al. (2005) & 103.0 \\
\hline 37061 & 0.52 & 3.42 & $103 \pm 6$ & $\sqrt{ }$ & Thorburn et al. (2003) & \\
\hline 46202 & 0.49 & 4.74 & $169 \pm 15$ & $\sqrt{ }$ & Thorburn et al. (2003) & 169.0 \\
\hline 47129 & 0.34 & 6.38 & $32.2 \pm 2.1$ & $x$ & Megier et al. (2005) & \\
\hline 48099 & 0.27 & 5.63 & $90 \pm 5$ & $\sqrt{ }$ & Thorburn et al. (2003) & 90.0 \\
\hline 122879 & 0.34 & 6.83 & $43.5 \pm 2.3$ & $\times$ & Megier et al. (2005) & 122.0 \\
\hline 122879 & 0.35 & 6.83 & 122.1 & $\sqrt{ }$ & Benvenuti \& Porceddu (1989) & \\
\hline 123008 & 0.63 & 4.94 & 258.3 & $\sqrt{ }$ & Benvenuti \& Porceddu (1989) & 258.0 \\
\hline 142096 & 0.19 & 4.46 & $14.5 \pm 1.4$ & $\times$ & Megier et al. (2005) & \\
\hline 143275 & 0.17 & 5.27 & 16 & $\sqrt{ }$ & Wszolk \& Godlowski (2003) & 16.0 \\
\hline 144217 & 0.19 & 6.49 & 20 & $\sqrt{ }$ & Wszolk \& Godlowski (2003) & 20.0 \\
\hline 144470 & 0.22 & 6.62 & 60 & $\sqrt{ }$ & Herbig $(1975)$ & 63.7 \\
\hline 144470 & 0.19 & 6.62 & $21.7 \pm 0.9$ & $x$ & Megier et al. (2005) & \\
\hline 144470 & 0.19 & 6.62 & 93.1 & $\sqrt{ }$ & Benvenuti \& Porceddu (1989) & \\
\hline 144470 & 0.22 & 6.62 & 38 & $\sqrt{ }$ & Wszolk \& Godlowski (2003) & \\
\hline 147933 & 0.47 & 5.62 & 35 & $\sqrt{ }$ & Herbig (1975) & \\
\hline 149757 & 0.32 & 6.63 & $36 \pm 5$ & $\sqrt{ }$ & Thorburn et al. (2003) & 27.0 \\
\hline 149757 & 0.29 & 6.63 & $14.5 \pm 0.8$ & $\times$ & Megier et al. (2005) & \\
\hline 149757 & 0.32 & 6.63 & 18 & $\sqrt{ }$ & Wszolk \& Godlowski (2003) & \\
\hline 152233 & 0.45 & 5.76 & 107.1 & $\sqrt{ }$ & Benvenuti \& Porceddu (1989) & 107.0 \\
\hline 152247 & 0.44 & 5.80 & 101.2 & $\sqrt{ }$ & Benvenuti \& Porceddu (1989) & 101.0 \\
\hline 152248 & 0.45 & 5.24 & 90.9 & $\sqrt{ }$ & Benvenuti \& Porceddu (1989) & 90.0 \\
\hline 152249 & 0.46 & 6.13 & 115.9 & $\sqrt{ }$ & Benvenuti \& Porceddu (1989) & 115.0 \\
\hline 154445 & 0.39 & 7.34 & $35.6 \pm 3.2$ & $\times$ & Megier et al. (2005) & - \\
\hline 162978 & 0.35 & 5.32 & 158.9 & $\sqrt{ }$ & Benvenuti \& Porceddu (1989) & 158.0 \\
\hline 164794 & 0.33 & 6.31 & 77.9 & $\sqrt{ }$ & Benvenuti \& Porceddu (1989) & 77.0 \\
\hline 165052 & 0.44 & 5.68 & 92.8 & $\sqrt{ }$ & Benvenuti \& Porceddu (1989) & 92.0 \\
\hline 166937 & 0.26 & 6.23 & $34.8 \pm 0.8$ & $x$ & Megier et al. (2005) & 82.0 \\
\hline 166937 & 0.25 & 6.23 & 86 & $\sqrt{ }$ & Benvenuti \& Porceddu (1989) & \\
\hline 166937 & 0.24 & 6.23 & 78 & $\sqrt{ }$ & Herbig (1975) & \\
\hline 167971 & 1.08 & 5.46 & $241 \pm 10$ & $\sqrt{ }$ & Thorburn et al. (2003) & 312.0 \\
\hline 167971 & 1.08 & 5.46 & 383 & $\sqrt{ }$ & Herbig (1975) & \\
\hline 167971 & 1.05 & 5.46 & $84.9 \pm 5.4$ & $x$ & Megier et al. (2005) & \\
\hline 168076 & 0.81 & 4.81 & 208.2 & $\sqrt{ }$ & Benvenuti \& Porceddu (1989) & 213.5 \\
\hline 168076 & 0.78 & 4.81 & $219 \pm 15$ & $\sqrt{ }$ & Thorburn et al. (2003) & \\
\hline 183143 & 1.27 & 6.13 & $350 \pm 11$ & $\sqrt{ }$ & Thorburn et al. (2003) & 268.7 \\
\hline 183143 & 1.27 & 6.13 & 250 & $\sqrt{ }$ & Wszolk \& Godlowski (2003) & \\
\hline 183143 & 1.27 & 6.13 & $206.2 \pm 1.5$ & $\sqrt{ }$ & Hobbs et al. (2009) & \\
\hline 185418 & 0.50 & 6.67 & $111 \pm 6$ & $\sqrt{ }$ & Thorburn et al. (2003) & 111.0 \\
\hline 190603 & 0.71 & 4.88 & 264 & $\sqrt{ }$ & Herbig (1975) & 264.0 \\
\hline 190603 & 0.71 & 4.88 & $82.8 \pm 3.7$ & $\times$ & Megier et al. (2005) & \\
\hline
\end{tabular}


Table 7-Continued

\begin{tabular}{|c|c|c|c|c|c|c|}
\hline $\mathrm{HD} / \mathrm{BD}$ & $\begin{array}{c}E(B-V) \\
\quad(\mathrm{mag})\end{array}$ & $\begin{array}{c}W_{2175} / E(B-V) \\
\left(\mu \mathrm{m}^{-1}\right)\end{array}$ & $\begin{array}{c}W_{6203} \\
(\mathrm{~m} \AA)\end{array}$ & $\begin{array}{c}\text { Adopt " } \sqrt{ } " \\
\text { or Reject "X" }\end{array}$ & Sources & $\begin{array}{c}W_{6203}(\mathrm{~m} \AA) \\
\text { This Work }\end{array}$ \\
\hline 198478 & 0.53 & 5.44 & 190 & $\sqrt{ }$ & Herbig (1975) & 160.0 \\
\hline 198478 & 0.54 & 5.44 & $130 \pm 7$ & $\sqrt{ }$ & Thorburn et al. (2003) & \\
\hline 198478 & 0.54 & 5.44 & $52.2 \pm 1.5$ & $x$ & Megier et al. (2005) & \\
\hline 199478 & 0.47 & 6.42 & 107 & $\sqrt{ }$ & Herbig (1975) & 107.0 \\
\hline 199579 & 0.35 & 4.60 & $26.1 \pm 1.9$ & $\times$ & Megier et al. (2005) & 46.5 \\
\hline 199579 & 0.37 & 4.60 & $53 \pm 2$ & $\sqrt{ }$ & Thorburn et al. (2003) & \\
\hline 199579 & 0.36 & 4.60 & 40 & $\sqrt{ }$ & Herbig (1975) & \\
\hline 203938 & 0.74 & 5.70 & $151 \pm 5$ & $\sqrt{ }$ & Thorburn et al. (2003) & 151.0 \\
\hline 203938 & 0.70 & 5.70 & $58.2 \pm 3.7$ & $\times$ & Megier et al. (2005) & \\
\hline 204827 & 1.11 & 4.25 & $116 \pm 4$ & $\sqrt{ }$ & Thorburn et al. (2003) & 83.9 \\
\hline 204827 & 1.11 & 4.25 & $51.7 \pm 1.7$ & $\sqrt{ }$ & Hobbs et al. (2008) & \\
\hline 206165 & 0.46 & 6.07 & $42.8 \pm 1.4$ & $\sqrt{ }$ & Megier et al. (2005) & 62.9 \\
\hline 206165 & 0.47 & 6.07 & $86 \pm 6$ & $\sqrt{ }$ & Thorburn et al. (2003) & \\
\hline 206165 & 0.47 & 6.07 & 60 & $\sqrt{ }$ & Wszolk \& Godlowski (2003) & \\
\hline 206267 & 0.53 & 4.96 & $103 \pm 5$ & $\sqrt{ }$ & Thorburn et al. (2003) & 89.0 \\
\hline 206267 & 0.50 & 4.96 & $44.2 \pm 1.5$ & $\times$ & Megier et al. (2005) & \\
\hline 206267 & 0.53 & 4.96 & 75 & $\sqrt{ }$ & Wszolk \& Godlowski (2003) & \\
\hline 207198 & 0.62 & 5.23 & $111 \pm 5$ & $\sqrt{ }$ & Thorburn et al. (2003) & 93.5 \\
\hline 207198 & 0.54 & 5.23 & $42.6 \pm 2.2$ & $x$ & Megier et al. (2005) & \\
\hline 207198 & 0.62 & 5.23 & 76 & $\sqrt{ }$ & Wszolk \& Godlowski (2003) & \\
\hline 210121 & 0.40 & 2.74 & $27.5 \pm 4$ & $\sqrt{ }$ & Thorburn et al. (2003) & 27.5 \\
\hline 303308 & 0.46 & 4.86 & 98.9 & $\sqrt{ }$ & Benvenuti \& Porceddu (1989) & 98.0 \\
\hline+631964 & 0.96 & 5.68 & $159.3 \pm 7.0$ & $\times$ & Megier et al. (2005) & 313.0 \\
\hline+631964 & 1.00 & 5.68 & $313 \pm 15$ & $\sqrt{ }$ & Thorburn et al. (2003) & \\
\hline
\end{tabular}


Table 8. Same as Table 3 but for the $\lambda 6269 / 6270 \AA$ DIB.

\begin{tabular}{|c|c|c|c|c|c|c|c|c|}
\hline $\mathrm{HD} / \mathrm{BD}$ & $\begin{array}{c}E(B-V) \\
\quad(\mathrm{mag})\end{array}$ & $\begin{array}{c}W_{2175} / E(B-V) \\
\left(\mu \mathrm{m}^{-1}\right)\end{array}$ & $\begin{array}{c}W_{6269} \\
(\mathrm{~m} \AA)\end{array}$ & $\begin{array}{c}\text { Adopt "V" } \\
\text { or Reject "X" }\end{array}$ & $\begin{array}{c}W_{6270} \\
(\mathrm{~m} \AA)\end{array}$ & $\begin{array}{c}\text { Adopt " } \sqrt{ } \text { " } \\
\text { or Reject "X" }\end{array}$ & Sources & $\begin{array}{c}W_{6269 / 6270}(\mathrm{~m} \AA \\
\text { This Work }\end{array}$ \\
\hline 2905 & 0.33 & 4.05 & - & $x$ & $63.0 \pm 4.4$ & $\sqrt{ }$ & Megier et al. (2005) & 59.0 \\
\hline 2905 & 0.33 & 4.05 & - & $x$ & $55 \pm 5$ & $\sqrt{ }$ & Snow et al. (2002) & \\
\hline 2905 & 0.32 & 4.05 & 174 & $x$ & - & $\times$ & Herbig (1975) & \\
\hline 15558 & 0.75 & 5.78 & $137.3 \pm 7.5$ & $\sqrt{ }$ & - & $x$ & Désert et al. (1995) & 137.3 \\
\hline 21291 & 0.41 & 7.31 & 115 & 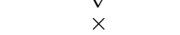 & - & $\hat{x}$ & Herbig (1975) & 48.8 \\
\hline 21291 & 0.42 & 7.31 & - & $x$ & $48.8 \pm 6.6$ & $\sqrt{ }$ & Megier et al. (2005) & \\
\hline 21483 & 0.56 & 4.07 & - & $\hat{x}$ & $59 \pm 8$ & $\sqrt{ }$ & Thorburn et al. (2003) & 59.0 \\
\hline 29647 & 1.00 & 3.82 & - & $x$ & $14.6 \pm 4$ & $\sqrt{ }$ & Thorburn et al. (2003) & 14.6 \\
\hline 30614 & 0.26 & 5.36 & - & $\hat{x}$ & $12.1 \pm 1.1$ & $x$ & Megier et al. (2005) & 56.0 \\
\hline 30614 & 0.32 & 5.36 & $63.0 \pm 9.9$ & $\checkmark$ & - & $x$ & Désert et al. (1995) & \\
\hline 30614 & 0.30 & 5.36 & - & $x$ & $49 \pm 6$ & $\checkmark$ & Thorburn et al. (2003) & \\
\hline+31643 & 0.85 & 6.59 & $52.7 \pm 12.8$ & $\hat{v}$ & - & $x$ & Désert et al. (1995) & 52.7 \\
\hline 34078 & 0.52 & 6.00 & 172 & $x$ & - & $\hat{x}$ & Herbig (1975) & 53.8 \\
\hline 34078 & 0.52 & 6.00 & - & $x$ & $56 \pm 3$ & $\sqrt{ }$ & Thorburn et al. (2003) & \\
\hline 34078 & 0.52 & 6.00 & $57.2 \pm 6.8$ & $\hat{v}$ & - & $x$ & Désert et al. (1995) & \\
\hline 34078 & 0.49 & 6.00 & - & $x$ & $48.3 \pm 2.0$ & $\hat{\jmath}$ & Megier et al. (2005) & \\
\hline 36879 & 0.50 & 4.92 & $73.0 \pm 9.0$ & $\hat{v}$ & - & $x$ & Désert et al. (1995) & 73.0 \\
\hline 37061 & 0.52 & 3.42 & - & $\times$ & $30.5 \pm 6$ & $\sqrt{ }$ & Thorburn et al. (2003) & 30.5 \\
\hline 37367 & 0.43 & 7.15 & $107.1 \pm 15.1$ & $\hat{v}$ & - & $x$ & Désert et al. (1995) & 107.1 \\
\hline 37903 & 0.38 & 3.64 & $33.1 \pm 5.3$ & $\sqrt{ }$ & - & $x$ & Désert et al. (1995) & 26.0 \\
\hline 37903 & 0.35 & 3.64 & - & $x$ & $19 \pm 5$ & $\hat{v}$ & Thorburn et al. (2003) & \\
\hline 38131 & 0.51 & 6.61 & $138.2 \pm 16.3$ & $\sqrt{ }$ & - & $x$ & Désert et al. (1995) & 138.2 \\
\hline 41117 & 0.44 & 5.89 & - & $x$ & $62.5 \pm 2.4$ & $\hat{v}$ & Megier et al. (2005) & 85.3 \\
\hline 41117 & 0.45 & 5.89 & - & $x$ & $108 \pm 4$ & $\sqrt{ }$ & Thorburn et al. (2003) & \\
\hline 42087 & 0.36 & 6.52 & - & $\hat{x}$ & $64 \pm 4$ & $\sqrt{ }$ & Thorburn et al. (2003) & 64.0 \\
\hline 46202 & 0.49 & 4.74 & _- & $\hat{x}$ & $84 \pm 5$ & $\sqrt{ }$ & Thorburn et al. (2003) & 84.0 \\
\hline 47129 & 0.34 & 6.38 & - & $\hat{x}$ & $36.4 \pm 2.5$ & $\sqrt{ }$ & Megier et al. (2005) & 36.4 \\
\hline 48099 & 0.27 & 5.63 & - & $\hat{x}$ & $45 \pm 4$ & $\sqrt{ }$ & Thorburn et al. (2003) & 45.0 \\
\hline+60497 & 0.89 & 5.94 & $170.0 \pm 12.5$ & $\hat{v}$ & - & $x$ & Désert et al. (1995) & 170.0 \\
\hline 122879 & 0.34 & 6.83 & - & $\times$ & $56.4 \pm 4.6$ & $\sqrt{ }$ & Megier et al. (2005) & 67.8 \\
\hline 122879 & 0.35 & 6.83 & - & $\hat{x}$ & 79.1 & $\sqrt{ }$ & Benvenuti \& Porceddu (1989) & \\
\hline 123008 & 0.63 & 4.94 & - & $x$ & 115.3 & $\sqrt{ }$ & Benvenuti \& Porceddu (1989) & 115.3 \\
\hline 142096 & 0.19 & 4.46 & - & $\hat{x}$ & $13.6 \pm 2.1$ & $\sqrt{ }$ & Megier et al. (2005) & 13.6 \\
\hline 143275 & 0.17 & 5.27 & - & $\hat{x}$ & 10 & $\sqrt{ }$ & Wszolk \& Godlowski (2003) & 10.0 \\
\hline 144217 & 0.19 & 6.50 & - & $x$ & 28 & $\sqrt{ }$ & Wszolk \& Godlowski (2003) & 28.0 \\
\hline 144470 & 0.19 & 6.62 & - & $\hat{x}$ & $20.7 \pm 0.8$ & $\sqrt{ }$ & Megier et al. (2005) & 22.9 \\
\hline 144470 & 0.19 & 6.62 & - & $\hat{x}$ & 24 & $\sqrt{ }$ & Benvenuti \& Porceddu (1989) & \\
\hline 144470 & 0.22 & 6.62 & 108 & $x$ & - & $x$ & Herbig (1975) & \\
\hline 144470 & 0.22 & 6.62 & - & $\hat{x}$ & 24 & $\hat{v}$ & Wszolk \& Godlowski (2003) & \\
\hline 145502 & 0.24 & 6.25 & - & $\hat{x}$ & 19 & $\sqrt{ }$ & Wszolk \& Godlowski (2003) & 19.0 \\
\hline 147165 & 0.39 & 5.27 & 46 & $\hat{x}$ & - & $x$ & Herbig (1975) & 17.5 \\
\hline 147165 & 0.34 & 5.27 & - & $\hat{x}$ & $14.0 \pm 1.1$ & $\sqrt{ }$ & Megier et al. (2005) & \\
\hline 147165 & 0.41 & 5.27 & - & $x$ & 21 & $\checkmark$ & Wszolk \& Godlowski (2003) & \\
\hline 147888 & 0.47 & 5.87 & - & $\hat{x}$ & $72 \pm 3$ & $\sqrt{ }$ & Thorburn et al. (2003) & 46.0 \\
\hline 147888 & 0.47 & 5.87 & - & $\hat{x}$ & $20 \pm 4$ & $\sqrt{ }$ & Snow et al. (2002) & \\
\hline 147889 & 1.08 & 6.88 & 154 & $x$ & - & $x$ & Herbig (1975) & 56.0 \\
\hline 147889 & 1.07 & 6.88 & - & $\hat{x}$ & $56 \pm 3$ & $\hat{\sqrt{ }}$ & Thorburn et al. (2003) & \\
\hline 147933 & 0.45 & 5.62 & - & $\hat{x}$ & $20.0 \pm 1.3$ & $\sqrt{ }$ & Megier et al. (2005) & 25.0 \\
\hline 147933 & 0.48 & 5.62 & - & $\hat{x}$ & $30 \pm 5$ & $\sqrt{ }$ & Thorburn et al. (2003) & \\
\hline 149757 & 0.29 & 6.63 & - & $\hat{x}$ & $13.1 \pm 1.0$ & $\sqrt{ }$ & Megier et al. (2005) & 15.6 \\
\hline 149757 & 0.32 & 6.63 & - & $\hat{x}$ & 18 & $\sqrt{ }$ & Wszolk \& Godlowski (2003) & \\
\hline 152233 & 0.45 & 5.76 & _- & $\hat{x}$ & 59 & $\sqrt{ }$ & Benvenuti \& Porceddu (1989) & 59.0 \\
\hline 152247 & 0.44 & 5.80 & - & $\hat{x}$ & 54.1 & $\sqrt{ }$ & Benvenuti \& Porceddu (1989) & 54.1 \\
\hline 152248 & 0.45 & 5.24 & - & $x$ & 50 & $\sqrt{ }$ & Benvenuti \& Porceddu (1989) & 50.0 \\
\hline 152249 & 0.46 & 6.13 & - & $\hat{x}$ & 49.2 & $\sqrt{ }$ & Benvenuti \& Porceddu (1989) & 49.2 \\
\hline 154445 & 0.39 & 7.34 & - & $\hat{x}$ & $38.2 \pm 4.7$ & $\sqrt{ }$ & Megier et al. (2005) & 57.2 \\
\hline 154445 & 0.43 & 7.34 & $76.1 \pm 12.5$ & $\hat{\mathfrak{V}}$ & $\begin{array}{c}30.2 \pm .1 \\
-\end{array}$ & $\times$ & Désert et al. (1995) & \\
\hline 162978 & 0.35 & 5.32 & 10. & 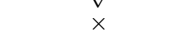 & 42 & $\hat{\sqrt{ }}$ & Benvenuti \& Porceddu (1989) & 42.0 \\
\hline 164794 & 0.33 & 6.31 & - & $x$ & 41 & $\sqrt{ }$ & Benvenuti \& Porceddu (1989) & 41.0 \\
\hline 165052 & 0.44 & 5.68 & - & $\hat{x}$ & 80.1 & $\sqrt{ }$ & Benvenuti \& Porceddu (1989) & 80.1 \\
\hline 166937 & 0.24 & 6.23 & 75 & $\hat{x}$ & - & $x$ & Herbig (1975) & 46.4 \\
\hline 166937 & 0.25 & 6.23 & - & $x$ & 49 & $\sqrt{ }$ & Benvenuti \& Porceddu (1989) & \\
\hline 166937 & 0.26 & 6.23 & - & $\hat{x}$ & $52.8 \pm 1.3$ & $\sqrt{ }$ & Megier et al. (2005) & \\
\hline 166937 & 0.51 & 6.23 & $37.2 \pm 3.6$ & $\hat{\jmath}$ & - & $\times$ & Désert et al. (1995) & \\
\hline 167971 & 1.08 & 5.46 & 246 & $x$ & - & $x$ & Herbig (1975) & 155.6 \\
\hline 167971 & 1.05 & 5.46 & - & $x$ & $166.2 \pm 10.5$ & $\sqrt{ }$ & Megier et al. (2005) & \\
\hline
\end{tabular}


Table 8-Continued

\begin{tabular}{|c|c|c|c|c|c|c|c|c|}
\hline $\mathrm{HD} / \mathrm{BD}$ & $\begin{array}{c}E(B-V) \\
\quad(\mathrm{mag})\end{array}$ & $\begin{array}{c}W_{2175} / E(B-V) \\
\left(\mu \mathrm{m}^{-1}\right)\end{array}$ & $\begin{array}{c}W_{6269} \\
(\mathrm{~m} \AA)\end{array}$ & $\begin{array}{c}\text { Adopt " } \sqrt{ } \text { " } \\
\text { or Reject "X" }\end{array}$ & $\begin{array}{c}W_{6270} \\
(\mathrm{~m} \AA)\end{array}$ & $\begin{array}{c}\text { Adopt " } \sqrt{ } " \\
\text { or Reject "X" }\end{array}$ & Sources & $\begin{array}{c}W_{6269 / 6270}(\mathrm{~m} \AA) \\
\text { This Work }\end{array}$ \\
\hline 167971 & 1.08 & 5.46 & - & $\times$ & $145 \pm 8$ & $\sqrt{ }$ & Thorburn et al. (2003) & \\
\hline 168076 & 0.81 & 4.81 & - & $\times$ & 109.4 & $\times$ & Benvenuti \& Porceddu (1989) & 126.0 \\
\hline 168076 & 0.80 & 4.81 & $124.0 \pm 9.6$ & $\sqrt{ }$ & - & $x$ & Désert et al. (1995) & \\
\hline 168076 & 0.78 & 4.81 & - & $\times$ & $128 \pm 10$ & $\sqrt{ }$ & Thorburn et al. (2003) & \\
\hline 168112 & 1.04 & 5.44 & $117.5 \pm 11.4$ & $\sqrt{ }$ & - & $x$ & Désert et al. (1995) & 117.5 \\
\hline 183143 & 1.24 & 6.13 & $210.8 \pm 13.6$ & $\sqrt{ }$ & - & $x$ & Désert et al. (1995) & 206.3 \\
\hline 183143 & 1.27 & 6.13 & - & $\times$ & $268 \pm 10$ & $\times$ & Thorburn et al. (2003) & \\
\hline 183143 & 1.27 & 6.13 & - & $\times$ & $188 \pm 5$ & $\sqrt{ }$ & Snow et al. (2002) & \\
\hline 183143 & 1.27 & 6.13 & - & $\times$ & 220 & $\sqrt{ }$ & Wszolk \& Godlowski (2003) & \\
\hline 183143 & 1.27 & 6.13 & - & $x$ & $256.4 \pm 1.4$ & $x$ & Hobbs et al. (2009) & \\
\hline 185418 & 0.50 & 6.67 & - & $x$ & $108 \pm 5$ & $\sqrt{ }$ & Thorburn et al. (2003) & 108.0 \\
\hline 190603 & 0.71 & 4.88 & 191 & $\times$ & - & $x$ & Herbig (1975) & 64.2 \\
\hline 190603 & 0.71 & 4.88 & - & $\times$ & $67.2 \pm 5.3$ & $\sqrt{ }$ & Megier et al. (2005) & \\
\hline 190603 & 0.94 & 4.88 & $61.1 \pm 1.9$ & $\sqrt{ }$ & - & $\times$ & Désert et al. (1995) & \\
\hline 192281 & 0.73 & 5.78 & $73.0 \pm 7.3$ & $\sqrt{ }$ & - & $x$ & Désert et al. (1995) & 73.0 \\
\hline 193682 & 0.83 & 7.40 & $132.0 \pm 10.0$ & $\sqrt{ }$ & - & $\times$ & Désert et al. (1995) & 132.0 \\
\hline 198478 & 0.53 & 5.44 & 190 & $\times$ & - & $\times$ & Herbig (1975) & 79.2 \\
\hline 198478 & 0.54 & 5.44 & - & $\times$ & $94 \pm 7$ & $\sqrt{ }$ & Thorburn et al. (2003) & \\
\hline 198478 & 0.54 & 5.44 & - & $\times$ & $64.4 \pm 0.4$ & $\sqrt{ }$ & Megier et al. (2005) & \\
\hline 199216 & 0.73 & 5.89 & $67.2 \pm 3.7$ & $\sqrt{ }$ & - & $x$ & Désert et al. (1995) & 67.2 \\
\hline 199478 & 0.47 & 6.42 & 180 & $\sqrt{ }$ & - & $x$ & Herbig (1975) & 180.0 \\
\hline 199579 & 0.38 & 4.60 & $42.2 \pm 5.7$ & $\sqrt{ }$ & - & $x$ & Désert et al. (1995) & 32.5 \\
\hline 199579 & 0.36 & 4.60 & 30 & $\sqrt{ }$ & - & $\times$ & Herbig (1975) & \\
\hline 199579 & 0.37 & 4.60 & - & $\times$ & $28 \pm 4$ & $\sqrt{ }$ & Thorburn et al. (2003) & \\
\hline 199579 & 0.35 & 4.60 & - & $\times$ & $30.0 \pm 3.5$ & $\sqrt{ }$ & Megier et al. (2005) & \\
\hline 203938 & 0.70 & 5.70 & - & $\times$ & $75.1 \pm 5.9$ & $\sqrt{ }$ & Megier et al. (2005) & 78.6 \\
\hline 203938 & 0.74 & 5.70 & - & $\times$ & $82 \pm 4$ & $\sqrt{ }$ & Thorburn et al. (2003) & \\
\hline 204827 & 1.11 & 4.25 & - & $\times$ & $82 \pm 5$ & $\sqrt{ }$ & Thorburn et al. (2003) & 79.5 \\
\hline 204827 & 1.11 & 4.25 & - & $\times$ & $77.0 \pm 1.7$ & $\sqrt{ }$ & Hobbs et al. (2008) & \\
\hline 206165 & 0.47 & 6.07 & - & $x$ & $57 \pm 5$ & $\sqrt{ }$ & Thorburn et al. (2003) & 57.7 \\
\hline 206165 & 0.46 & 6.07 & - & $\times$ & $60.1 \pm 1.5$ & $\sqrt{ }$ & Megier et al. (2005) & \\
\hline 206165 & 0.47 & 6.07 & - & $\times$ & 56 & $\sqrt{ }$ & Wszolk \& Godlowski (2003) & \\
\hline 206267 & 0.50 & 4.96 & - & $x$ & $72.9 \pm 4.1$ & $\sqrt{ }$ & Megier et al. (2005) & 73.0 \\
\hline 206267 & 0.53 & 4.96 & - & $\times$ & $74 \pm 3$ & $\sqrt{ }$ & Thorburn et al. (2003) & \\
\hline 206267 & 0.53 & 4.96 & - & $\times$ & 72 & $\sqrt{ }$ & Wszolk \& Godlowski (2003) & \\
\hline 207198 & 0.62 & 5.23 & - & $\hat{x}$ & $58 \pm 3$ & $\sqrt{ }$ & Thorburn et al. (2003) & 56.7 \\
\hline 207198 & 0.54 & 5.23 & - & $x$ & $54.1 \pm 2.1$ & $\sqrt{ }$ & Megier et al. (2005) & \\
\hline 207198 & 0.62 & 5.23 & - & $\hat{x}$ & 58 & $\sqrt{ }$ & Wszolk \& Godlowski (2003) & \\
\hline 209339 & 0.37 & 6.74 & $69.9 \pm 1.9$ & $\sqrt{ }$ & - & $\times$ & Désert et al. (1995) & 69.9 \\
\hline 210121 & 0.40 & 2.74 & - & $\times$ & $6 \pm 2$ & $\sqrt{ }$ & Thorburn et al. (2003) & 6.0 \\
\hline 216532 & 0.87 & 6.50 & $148.8 \pm 13.9$ & $\sqrt{ }$ & - & $\times$ & Désert et al. (1995) & 148.8 \\
\hline 216898 & 0.88 & 6.22 & $112.6 \pm 11.4$ & $\sqrt{ }$ & - & $x$ & Désert et al. (1995) & 112.6 \\
\hline 217086 & 0.92 & 6.48 & $131.6 \pm 7.4$ & $\sqrt{ }$ & - & $\times$ & Désert et al. (1995) & 131.6 \\
\hline 239729 & 0.67 & 4.68 & $83.8 \pm 2.0$ & $\sqrt{ }$ & - & $x$ & Désert et al. (1995) & 83.8 \\
\hline 303308 & 0.46 & 4.86 & - & $\times$ & 99.8 & $\sqrt{ }$ & Benvenuti \& Porceddu (1989) & 99.8 \\
\hline+631964 & 1.00 & 5.68 & - & $\times$ & $135 \pm 4$ & $\sqrt{ }$ & Thorburn et al. (2003) & 132.9 \\
\hline+631964 & 0.96 & 5.68 & - & $\times$ & $130.7 \pm 7.9$ & $\sqrt{ }$ & Megier et al. (2005) & \\
\hline+631964 & 0.95 & 5.68 & $162.5 \pm 7.6$ & $\times$ & - & $\times$ & Désert et al. (1995) & \\
\hline
\end{tabular}


Table 9 . Same as Table 3 but for the $\lambda 6284 \AA$ DIB.

\begin{tabular}{|c|c|c|c|c|c|c|}
\hline $\mathrm{HD} / \mathrm{BD}$ & $\begin{array}{l}E(B-V) \\
\quad(\mathrm{mag})\end{array}$ & $\begin{array}{c}W_{2175} / E(B-V) \\
\left(\mu \mathrm{m}^{-1}\right)\end{array}$ & $\begin{array}{l}W_{6284} \\
(\mathrm{~m} \AA)\end{array}$ & $\begin{array}{c}\text { Adopt "V" } \\
\text { or Reject "X" }\end{array}$ & Sources & $\begin{array}{l}W_{6284}(\mathrm{~m} \AA) \\
\text { This Work }\end{array}$ \\
\hline 2905 & 0.33 & 4.05 & $301.0 \pm 11.0$ & $x$ & Megier et al. (2005) & 665.0 \\
\hline 2905 & 0.33 & 4.05 & $665 \pm 65$ & $\sqrt{ }$ & Friedman et al. (2010) & \\
\hline 15558 & 0.75 & 5.78 & $1407.0 \pm 75.0$ & $\sqrt{ }$ & Désert et al. (1995) & 1407.0 \\
\hline 21291 & 0.42 & 7.31 & $320.0 \pm 15.5$ & $x$ & Megier et al. (2005) & - \\
\hline 21483 & 0.56 & 4.07 & $397 \pm 45$ & $\sqrt{ }$ & Friedman et al. (2010) & 397 \\
\hline 27778 & 0.37 & 4.75 & $170 \pm 50$ & $\sqrt{ }$ & Friedman et al. (2010) & 170 \\
\hline 29647 & 1.00 & 3.82 & $95 \pm 25$ & $\sqrt{ }$ & Friedman et al. (2010) & 95 \\
\hline 30614 & 0.32 & 5.36 & $374.1 \pm 58.6$ & $\sqrt{ }$ & Désert et al. (1995) & 367.1 \\
\hline 30614 & 0.26 & 5.36 & $125.0 \pm 5.3$ & $\times$ & Megier et al. (2005) & \\
\hline 30614 & 0.30 & 5.36 & $360 \pm 60$ & $\sqrt{ }$ & Friedman et al. (2010) & \\
\hline+31643 & 0.85 & 6.59 & $746.3 \pm 34.0$ & $\sqrt{ }$ & Désert et al. (1995) & 746.3 \\
\hline 34078 & 0.52 & 6.00 & $646.9 \pm 74.9$ & $\sqrt{ }$ & Désert et al. (1995) & 578.5 \\
\hline 34078 & 0.52 & 6.00 & $510 \pm 80$ & $\sqrt{ }$ & Friedman et al. (2010) & \\
\hline 36879 & 0.50 & 4.92 & $962.0 \pm 115.5$ & $\sqrt{ }$ & Désert et al. (1995) & 962.0 \\
\hline 37022 & 0.34 & 2.47 & $468 \pm 70$ & $\sqrt{ }$ & Friedman et al. (2010) & 468.0 \\
\hline 37061 & 0.50 & 3.42 & $182.0 \pm 11.7$ & $\times$ & Megier et al. (2005) & 675.0 \\
\hline 37061 & 0.52 & 3.42 & $675 \pm 55$ & $\sqrt{ }$ & Friedman et al. (2010) & \\
\hline 37367 & 0.43 & 7.15 & $1038.9 \pm 144.9$ & $\sqrt{ }$ & Désert et al. (1995) & 1077.9 \\
\hline 37367 & 0.40 & 7.15 & $1117 \pm 60$ & $\sqrt{ }$ & Friedman et al. (2010) & \\
\hline 37903 & 0.38 & 3.64 & $413.8 \pm 65.4$ & $\sqrt{ }$ & Désert et al. (1995) & 458.4 \\
\hline 37903 & 0.35 & 3.64 & $503 \pm 70$ & $\sqrt{ }$ & Friedman et al. (2010) & \\
\hline 38087 & 0.29 & 6.03 & $325 \pm 45$ & $\sqrt{ }$ & Friedman et al. (2010) & 325.0 \\
\hline 38131 & 0.51 & 6.61 & $1115.9 \pm 127.0$ & $\sqrt{ }$ & Désert et al. (1995) & 1115.9 \\
\hline 40893 & 0.46 & 5.92 & $1030 \pm 75$ & $\sqrt{ }$ & Friedman et al. (2010) & 1030.0 \\
\hline 41117 & 0.44 & 5.89 & $323.8 \pm 6.6$ & $\times$ & Megier et al. (2005) & 760.0 \\
\hline 41117 & 0.45 & 5.89 & $760 \pm 100$ & $\sqrt{ }$ & Friedman et al. (2010) & \\
\hline 42087 & 0.36 & 6.52 & $675 \pm 70$ & $\sqrt{ }$ & Friedman et al. (2010) & 675.0 \\
\hline 46056 & 0.50 & 5.11 & $750 \pm 60$ & $\sqrt{ }$ & Friedman et al. (2010) & 750.0 \\
\hline 46202 & 0.49 & 4.74 & $935 \pm 70$ & $\sqrt{ }$ & Friedman et al. (2010) & 935.0 \\
\hline 47129 & 0.36 & 6.38 & 150 & $\times$ & Seab \& Snow (1984) & 550.0 \\
\hline 47129 & 0.34 & 6.38 & $161.7 \pm 4.4$ & $x$ & Megier et al. (2005) & \\
\hline 47129 & 0.36 & 6.38 & $550 \pm 50$ & $\sqrt{ }$ & Friedman et al. (2010) & \\
\hline 48099 & 0.27 & 5.63 & 170 & $\times$ & Seab \& Snow (1984) & 595.0 \\
\hline 48099 & 0.27 & 5.63 & $595 \pm 50$ & $\sqrt{ }$ & Friedman et al. (2010) & \\
\hline 48434 & 0.28 & 6.31 & 180 & $x$ & Seab \& Snow (1984) & - \\
\hline+60497 & 0.89 & 5.94 & $1057.3 \pm 71.2$ & $\sqrt{ }$ & Désert et al. (1995) & 1057.3 \\
\hline 122879 & 0.35 & 6.83 & 672 & $\sqrt{ }$ & Benvenuti \& Porceddu (1989) & 672.0 \\
\hline 122879 & 0.34 & 6.83 & $725.0 \pm 25.0$ & $\times$ & Megier et al. (2005) & \\
\hline 123008 & 0.63 & 4.94 & 1217.8 & $\sqrt{ }$ & Benvenuti \& Porceddu (1989) & 1217.8 \\
\hline 142096 & 0.19 & 4.46 & $308.0 \pm 14.0$ & $\sqrt{ }$ & Megier et al. (2005) & 308.0 \\
\hline 143018 & 0.05 & 5.12 & $145 \pm 40$ & $\sqrt{ }$ & Friedman et al. (2010) & 145.0 \\
\hline 143275 & 0.17 & 5.27 & $250 \pm 25$ & $\sqrt{ }$ & Friedman et al. (2010) & 250.0 \\
\hline 144217 & 0.19 & 6.50 & $397 \pm 45$ & $\sqrt{ }$ & Friedman et al. (2010) & 397.0 \\
\hline 144470 & 0.19 & 6.62 & $124.5 \pm 1.4$ & $x$ & Megier et al. (2005) & 363.0 \\
\hline 144470 & 0.19 & 6.62 & 323 & $\sqrt{ }$ & Benvenuti \& Porceddu (1989) & \\
\hline 144470 & 0.22 & 6.62 & $403 \pm 40$ & $\sqrt{ }$ & Friedman et al. (2010) & \\
\hline 145502 & 0.24 & 6.25 & $421 \pm 40$ & $\sqrt{ }$ & Friedman et al. (2010) & 421.0 \\
\hline 147165 & 0.34 & 5.27 & $142.6 \pm 2.1$ & $\times$ & Megier et al. (2005) & 498.0 \\
\hline 147165 & 0.41 & 5.27 & $498 \pm 50$ & $\widehat{\sqrt{ }}$ & Friedman et al. (2010) & \\
\hline 147888 & 0.47 & 5.87 & $390 \pm 60$ & $\sqrt{ }$ & Friedman et al. (2010) & 390.0 \\
\hline 147889 & 1.08 & 6.88 & 90 & $\times$ & Seab \& Snow (1984) & 530.0 \\
\hline 147889 & 1.07 & 6.88 & $530 \pm 50$ & $\sqrt{ }$ & Friedman et al. (2010) & \\
\hline 147933 & 0.46 & 5.62 & 110 & $\times$ & Seab \& Snow (1984) & 426.0 \\
\hline 147933 & 0.45 & 5.62 & $176.4 \pm 2.8$ & $\times$ & Megier et al. (2005) & \\
\hline 147933 & 0.48 & 5.62 & $426 \pm 80$ & $\sqrt{ }$ & Friedman et al. (2010) & \\
\hline 149757 & 0.29 & 6.63 & $68.2 \pm 2.0$ & $\times$ & Megier et al. (2005) & 175.0 \\
\hline 149757 & 0.32 & 6.63 & $175 \pm 35$ & $\sqrt{ }$ & Friedman et al. (2010) & \\
\hline 152233 & 0.45 & 5.76 & 446.9 & $\sqrt{ }$ & Benvenuti \& Porceddu (1989) & 446.9 \\
\hline 152247 & 0.44 & 5.80 & 356.8 & $\sqrt{ }$ & Benvenuti \& Porceddu (1989) & 356.8 \\
\hline 152248 & 0.45 & 5.24 & 385.2 & $\sqrt{ }$ & Benvenuti \& Porceddu (1989) & 385.2 \\
\hline 152249 & 0.46 & 6.13 & 523.9 & $\sqrt{ }$ & Benvenuti \& Porceddu (1989) & 523.9 \\
\hline 154445 & 0.43 & 7.34 & $458.8 \pm 74.8$ & $\sqrt{ }$ & Désert et al. (1995) & 458.8 \\
\hline 162978 & 0.35 & 5.32 & 399 & $\times$ & Benvenuti \& Porceddu (1989) & 558.0 \\
\hline 162978 & 0.35 & 5.32 & $558 \pm 60$ & $\sqrt{ }$ & Friedman et al. (2010) & \\
\hline 164794 & 0.33 & 6.31 & 352.1 & $\sqrt{ }$ & Benvenuti \& Porceddu (1989) & 352.1 \\
\hline 165052 & 0.44 & 5.68 & 464.2 & $\sqrt{ }$ & Benvenuti \& Porceddu (1989) & 464.2 \\
\hline
\end{tabular}


Table 9-Continued

\begin{tabular}{|c|c|c|c|c|c|c|}
\hline $\mathrm{HD} / \mathrm{BD}$ & $\begin{array}{c}E(B-V) \\
\quad(\mathrm{mag})\end{array}$ & $\begin{array}{c}W_{2175} / E(B-V) \\
\left(\mu \mathrm{m}^{-1}\right)\end{array}$ & $\begin{array}{c}W_{6284} \\
(\mathrm{~m} \AA)\end{array}$ & $\begin{array}{l}\text { Adopt “ } " \\
\text { or Reject "X" }\end{array}$ & Sources & $\begin{array}{c}W_{6284}(\mathrm{~m} \AA) \\
\text { This Work }\end{array}$ \\
\hline 166937 & 0.25 & 6.23 & 628 & $\sqrt{ }$ & Benvenuti \& Porceddu (1989) & 754.7 \\
\hline 166937 & 0.26 & 6.23 & $319.5 \pm 2.5$ & $\times$ & Megier et al. (2005) & \\
\hline 166937 & 0.51 & 6.23 & $851.2 \pm 83.6$ & $\sqrt{ }$ & Désert et al. (1995) & \\
\hline 166937 & 0.25 & 6.23 & $785 \pm 70$ & $\sqrt{ }$ & Friedman et al. (2010) & \\
\hline 167771 & 0.42 & 4.60 & 160 & $\times$ & Seab \& Snow (1984) & 1450.0 \\
\hline 167971 & 1.05 & 5.46 & $650.5 \pm 19.6$ & $x$ & Megier et al. (2005) & \\
\hline 167971 & 1.08 & 5.46 & $1450 \pm 200$ & $\sqrt{ }$ & Friedman et al. (2010) & \\
\hline 168076 & 0.81 & 4.81 & 899.1 & $\sqrt{ }$ & Benvenuti \& Porceddu (1989) & 1033.4 \\
\hline 168076 & 0.80 & 4.81 & $1111.2 \pm 83.2$ & $\sqrt{ }$ & Désert et al. (1995) & \\
\hline 168076 & 0.78 & 4.81 & $1090 \pm 150$ & $\sqrt{ }$ & Friedman et al. (2010) & \\
\hline 168112 & 1.04 & 5.44 & $1335.4 \pm 115.4$ & $\sqrt{ }$ & Désert et al. (1995) & 1335.4 \\
\hline 183143 & 1.24 & 6.13 & $1869.9 \pm 120.3$ & $\sqrt{ }$ & Désert et al. (1995) & 1888.0 \\
\hline 183143 & 1.28 & 6.13 & 710 & $\times$ & Seab \& Snow (1984) & \\
\hline 183143 & 1.27 & 6.13 & $1910 \pm 30$ & $\sqrt{ }$ & Friedman et al. (2010) & \\
\hline 183143 & 1.27 & 6.13 & $1884.2 \pm 4.2$ & $\sqrt{ }$ & Hobbs et al. (2009) & \\
\hline 185418 & 0.50 & 6.67 & $640 \pm 50$ & $\sqrt{ }$ & Friedman et al. (2010) & 640.0 \\
\hline 190603 & 0.94 & 4.88 & $1270.9 \pm 27.3$ & $\sqrt{ }$ & Désert et al. (1995) & 1270.9 \\
\hline 190603 & 0.71 & 4.88 & $473.0 \pm 13.2$ & $\times$ & Megier et al. (2005) & \\
\hline 192281 & 0.73 & 5.78 & $773.1 \pm 74.5$ & $\sqrt{ }$ & Désert et al. (1995) & 773.1 \\
\hline 193682 & 0.83 & 7.40 & $229.1 \pm 16.6$ & $\sqrt{ }$ & Désert et al. (1995) & 229.1 \\
\hline 198478 & 0.54 & 5.44 & $394.0 \pm 4.9$ & $\times$ & Megier et al. (2005) & 919.0 \\
\hline 198478 & 0.54 & 5.44 & $919 \pm 60$ & $\sqrt{ }$ & Friedman et al. (2010) & \\
\hline 199216 & 0.73 & 5.89 & $573.8 \pm 9.5$ & $\sqrt{ }$ & Désert et al. (1995) & 573.8 \\
\hline 199579 & 0.37 & 4.60 & 80 & $\times$ & Seab \& Snow (1984) & 295.1 \\
\hline 199579 & 0.35 & 4.60 & $73.2 \pm 3.0$ & $\times$ & Megier et al. (2005) & \\
\hline 199579 & 0.38 & 4.60 & $275.1 \pm 36.1$ & $\sqrt{ }$ & Désert et al. (1995) & \\
\hline 199579 & 0.37 & 4.60 & $315 \pm 50$ & $\sqrt{ }$ & Friedman et al. (2010) & \\
\hline 203938 & 0.70 & 5.70 & $337.4 \pm 7.8$ & $\times$ & Megier et al. (2005) & 936.0 \\
\hline 203938 & 0.74 & 5.70 & $936 \pm 60$ & $\sqrt{ }$ & Friedman et al. (2010) & \\
\hline 204827 & 1.11 & 4.25 & $518 \pm 60$ & $\sqrt{ }$ & Friedman et al. (2010) & 488.9 \\
\hline 204827 & 1.11 & 4.25 & $459.7 \pm 6.9$ & $\sqrt{ }$ & Hobbs et al. (2008) & \\
\hline 206165 & 0.46 & 6.07 & $243.1 \pm 1.2$ & $\times$ & Megier et al. (2005) & 486.0 \\
\hline 206165 & 0.47 & 6.07 & $486 \pm 60$ & $\sqrt{ }$ & Friedman et al. (2010) & \\
\hline 206267 & 0.50 & 4.96 & $199.2 \pm 4.6$ & $\times$ & Megier et al. (2005) & 544.0 \\
\hline 206267 & 0.53 & 4.96 & $544 \pm 45$ & $\sqrt{ }$ & Friedman et al. (2010) & \\
\hline 207198 & 0.54 & 5.23 & $168.5 \pm 2.8$ & $x$ & Megier et al. (2005) & 543.0 \\
\hline 207198 & 0.62 & 5.23 & $543 \pm 40$ & $\sqrt{ }$ & Friedman et al. (2010) & \\
\hline 209339 & 0.36 & 6.74 & 220 & $\times$ & Seab \& Snow (1984) & 713.0 \\
\hline 209339 & 0.37 & 6.74 & $713.0 \pm 19.2$ & $\sqrt{ }$ & Désert et al. (1995) & \\
\hline 210121 & 0.40 & 2.74 & $146 \pm 50$ & $\sqrt{ }$ & Friedman et al. (2010) & 146.0 \\
\hline 216532 & 0.87 & 6.50 & $1259.8 \pm 115.7$ & $\sqrt{ }$ & Désert et al. (1995) & 1259.8 \\
\hline 216898 & 0.88 & 6.22 & $1223.2 \pm 125.0$ & $\sqrt{ }$ & Désert et al. (1995) & 1223.2 \\
\hline 217086 & 0.92 & 6.48 & $1258.6 \pm 68.1$ & $\sqrt{ }$ & Désert et al. (1995) & 1258.6 \\
\hline 239729 & 0.67 & 4.68 & $393.3 \pm 6.0$ & $\sqrt{ }$ & Désert et al. (1995) & 393.3 \\
\hline 242908 & 0.62 & 5.13 & $1029.8 \pm 50.2$ & $\sqrt{ }$ & Désert et al. (1995) & 1029.8 \\
\hline 303308 & 0.46 & 4.86 & 584.7 & $\sqrt{ }$ & Benvenuti \& Porceddu (1989) & 584.7 \\
\hline+631964 & 0.95 & 5.68 & $1535.2 \pm 34.2$ & $\sqrt{ }$ & Désert et al. (1995) & 1457.6 \\
\hline+631964 & 0.96 & 5.68 & $490.9 \pm 11.6$ & $\times$ & Megier et al. (2005) & \\
\hline+631964 & 1.00 & 5.68 & $1380 \pm 200$ & $\sqrt{ }$ & Friedman et al. (2010) & \\
\hline
\end{tabular}


Table 10. Same as Table 3 but for the $\lambda 6376 / 6379 \AA$ DIB.

\begin{tabular}{|c|c|c|c|c|c|c|c|c|}
\hline $\mathrm{HD} / \mathrm{BD}$ & $\begin{array}{c}E(B-V) \\
\quad(\mathrm{mag})\end{array}$ & $\begin{array}{c}W_{2175} / E(B-V) \\
\left(\mu \mathrm{m}^{-1}\right)\end{array}$ & $\begin{array}{c}W_{6376} \\
(\mathrm{~m} \AA)\end{array}$ & $\begin{array}{c}\text { Adopt "V" } \\
\text { or Reject "X" }\end{array}$ & $\begin{array}{c}W_{6379} \\
(\mathrm{~m} \AA)\end{array}$ & $\begin{array}{c}\text { Adopt "V" } \\
\text { or Reject "X" }\end{array}$ & Sources & $\begin{array}{c}W_{6376 / 6379}(\mathrm{~m} \AA) \\
\text { This Work }\end{array}$ \\
\hline 2905 & 0.33 & 4.05 & - & $x$ & $612 \pm 45$ & $x$ & Snow et al. (2002) & 74.8 \\
\hline 2905 & 0.32 & 4.05 & 67 & $x$ & 115 & $x$ & Herbig (1975) & \\
\hline 2905 & 0.33 & 4.05 & $24.6 \pm 1.3$ & $\checkmark$ & $50.2 \pm 0.9$ & $\checkmark$ & Megier et al. (2005) & \\
\hline 15570 & 1.02 & 5.52 & - & $\times$ & $89.8 \pm 20.4$ & $x^{v}$ & Sonnentrucher et al. (1997) & \\
\hline 21291 & 0.41 & 7.31 & - & $\hat{x}$ & 69 & $\hat{x}$ & Herbig (1975) & 43.5 \\
\hline 21291 & 0.43 & 7.31 & - & $x$ & 31.8 & $x$ & Cox et al. (2007) & \\
\hline 21291 & 0.42 & 7.31 & $15.5 \pm 2.4$ & $\sqrt{ }$ & $28.0 \pm 2.0$ & $\hat{v}$ & Megier et al. (2005) & \\
\hline 21483 & 0.56 & 4.07 & $23 \pm 2$ & $\sqrt{ }$ & $51.5 \pm 1$ & $\sqrt{v}$ & Thorburn et al. (2003) & 74.5 \\
\hline 27778 & 0.33 & 4.75 & - & $\times$ & $20.1 \pm 1.0$ & $x$ & Sonnentrucher et al. (1997) & 27.5 \\
\hline 27778 & 0.37 & 4.75 & $7.5 \pm 1$ & $\checkmark$ & $20 \pm 1$ & $\sqrt{ }$ & Thorburn et al. (2003) & \\
\hline 29647 & 1.03 & 3.82 & - & $x$ & $14.4 \pm 3.1$ & $x$ & Sonnentrucher et al. (1997) & 29.9 \\
\hline 29647 & 1.00 & 3.82 & $11.4 \pm 2$ & $\hat{v}$ & $18.5 \pm 1$ & $\hat{v}$ & Thorburn et al. (2003) & \\
\hline 30614 & 0.30 & 5.36 & $14 \pm 1.5$ & $\sqrt{ }$ & $34.5 \pm 1$ & 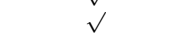 & Thorburn et al. (2003) & 45.1 \\
\hline 30614 & 0.32 & 5.36 & - & $x$ & $34.9 \pm 5.1$ & $x$ & Sonnentrucher et al. (1997) & \\
\hline 30614 & 0.26 & 5.36 & $8.6 \pm 1.0$ & $\sqrt{ }$ & $33.0 \pm 1.1$ & $\hat{v}$ & Megier et al. (2005) & \\
\hline 34078 & 0.52 & 6.00 & ${ }_{-}^{0.0}$ & $x_{x}$ & 50 & $\begin{array}{c}v \\
x\end{array}$ & Herbig (1975) & 26.4 \\
\hline 34078 & 0.52 & 6.00 & $9 \pm 1$ & $\sqrt{ }$ & $18 \pm 1$ & $\hat{v}$ & Thorburn et al. (2003) & \\
\hline 34078 & 0.49 & 6.00 & $9 \pm 1$ & $\checkmark$ & $16.8 \pm 1.0$ & $\sqrt{v}$ & Megier et al. (2005) & \\
\hline 37022 & 0.36 & 2.47 & - & $\times$ & $16.2 \pm 9.0$ & $\mathrm{x}$ & Sonnentrucher et al. (1997) & \\
\hline 37022 & 0.34 & 2.47 & - & $\hat{x}$ & $460 \pm 60$ & $\hat{x}$ & Snow et al. $(2002)$ & \\
\hline 37061 & 0.50 & 3.42 & - & $\hat{x}$ & $30.0 \pm 7.0$ & $\hat{x}$ & Sonnentrucher et al. (1997) & 14.3 \\
\hline 37061 & 0.52 & 3.42 & $7.1 \pm 2$ & $\checkmark$ & $7.2 \pm 1.5$ & $\sqrt{ }$ & Thorburn et al. (2003) & \\
\hline 37903 & 0.35 & 3.64 & $<4$ & $\times$ & $3.5 \pm 1$ & $x$ & Thorburn et al. (2003) & \\
\hline 41117 & 0.45 & 5.89 & $29 \pm 1$ & $\hat{v}$ & $136 \pm 5$ & $\hat{\sqrt{ }}$ & Thorburn et al. (2003) & 165.0 \\
\hline 41117 & 0.44 & 5.89 & $29.2 \pm 1.8$ & $\times$ & - & $x$ & Megier et al. (2005) & \\
\hline 42087 & 0.35 & 6.52 & $17.5 \pm 1.1$ & $\checkmark$ & $37.0 \pm 1.2$ & $\sqrt{ }$ & Megier et al. (2005) & 72.8 \\
\hline 42087 & 0.36 & 6.52 & $23 \pm 4$ & $\sqrt{ }$ & $68 \pm 3$ & 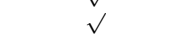 & Thorburn et al. (2003) & \\
\hline 46202 & 0.49 & 4.74 & $27 \pm 3$ & $\sqrt{ }$ & $56 \pm 2$ & v & Thorburn et al. (2003) & 83.0 \\
\hline 47129 & 0.34 & 6.38 & $19.9 \pm 1.5$ & $\sqrt{ }$ & $30.0 \pm 1.2$ & $\sqrt{ }$ & Megier et al. (2005) & 49.9 \\
\hline 48099 & 0.27 & 5.63 & $12.8 \pm 1.5$ & $\sqrt{ }$ & $19.8 \pm 0.8$ & $\sqrt{ }$ & Thorburn et al. (2003) & 32.6 \\
\hline 122879 & 0.34 & 6.83 & $17.6 \pm 2.5$ & $\sqrt{ }$ & $23.0 \pm 2.0$ & $\checkmark$ & Megier et al. (2005) & 40.6 \\
\hline 142096 & 0.19 & 4.46 & - & $x_{x}$ & $4.5 \pm 0.8$ & $x^{v}$ & Megier et al. (2005) & \\
\hline 143275 & 0.17 & 5.27 & 4 & $\hat{v}$ & 10 & $\hat{\jmath}$ & Wszolk \& Godlowski (2003) & 14.0 \\
\hline 144217 & 0.19 & 6.49 & - & $x$ & $14.1 \pm 1.1$ & $\mathrm{x}$ & Sonnentrucher et al. (1997) & 17.0 \\
\hline 144217 & 0.19 & 6.50 & 4 & $\sqrt{ }$ & 13 & $\hat{v}$ & Wszolk \& Godlowski (2003) & \\
\hline 144470 & 0.19 & 6.62 & $7.4 \pm 0.5$ & $\sqrt{ }$ & $23.3 \pm 0.6$ & v & Megier et al. (2005) & 33.4 \\
\hline 144470 & 0.22 & 6.62 & 10 & $\sqrt{ }$ & 26 & $\checkmark$ & Wszolk \& Godlowski (2003) & \\
\hline 145502 & 0.24 & 6.25 & - & $x$ & $32.0 \pm 7.0$ & $x$ & Sonnentrucher et al. (1997) & 39.0 \\
\hline 145502 & 0.24 & 6.25 & 10 & $\hat{v}$ & 29 & $\hat{\jmath}$ & Wszolk \& Godlowski (2003) & \\
\hline 147165 & 0.39 & 5.27 & - & $x$ & 45 & $x$ & Herbig (1975) & 32.8 \\
\hline 147165 & 0.34 & 5.27 & $9.5 \pm 0.5$ & $\hat{v}$ & $20.1 \pm 0.3$ & $\hat{v}$ & Megier et al. (2005) & \\
\hline 147165 & 0.41 & 5.27 & 11 & $\sqrt{ }$ & 25 & v & Wszolk \& Godlowski (2003) & \\
\hline 147888 & 0.47 & 5.87 & - & $\times$ & $415 \pm 40$ & $x$ & Snow et al. (2002) & 54.0 \\
\hline 147888 & 0.47 & 5.87 & $21 \pm 1$ & $\checkmark$ & $33 \pm 1$ & $\hat{\sqrt{ }}$ & Thorburn et al. (2003) & \\
\hline 147889 & 1.08 & 6.88 & 105 & $\times$ & 124 & $x$ & Herbig (1975) & 157.0 \\
\hline 147889 & 1.07 & 6.88 & $64 \pm 2$ & $\checkmark$ & $93 \pm 2$ & $\sqrt{ }$ & Thorburn et al. (2003) & \\
\hline 147933 & 0.47 & 5.62 & 23 & $\times$ & 62 & $x$ & Herbig (1975) & 41.4 \\
\hline 147933 & 0.45 & 5.62 & $11.6 \pm 0.8$ & $\hat{v}$ & $25.9 \pm 1.0$ & $\hat{\mathfrak{v}}$ & Megier et al. (2005) & \\
\hline 147933 & 0.48 & 5.62 & $17.3 \pm 2$ & $\sqrt{ }$ & $28 \pm 1$ & $\checkmark$ & Thorburn et al. (2003) & \\
\hline 149757 & 0.32 & 6.63 & - & $x$ & $20.2 \pm 2.2$ & $x$ & Sonnentrucher et al. (1997) & 29.8 \\
\hline 149757 & 0.29 & 6.63 & $3.5 \pm 0.3$ & $\hat{x}$ & $18.7 \pm 0.5$ & $\hat{x}$ & Megier et al. (2005) & \\
\hline 149757 & 0.32 & 6.63 & $12.5 \pm 1$ & $\checkmark$ & $20 \pm 1$ & $\hat{\sqrt{ }}$ & Thorburn et al. (2003) & \\
\hline 149757 & 0.32 & 6.63 & 7 & $\sqrt{ }$ & 20 & $\checkmark$ & Wszolk \& Godlowski (2003) & \\
\hline 154445 & 0.39 & 7.34 & $20.0 \pm 2.3$ & $\checkmark$ & $43.6 \pm 1.7$ & $\checkmark$ & Megier et al. (2005) & 63.6 \\
\hline 154445 & 0.42 & 7.34 & - & $\times$ & $33.2 \pm 5.0$ & $x$ & Sonnentrucher et al. (1997) & \\
\hline 166937 & 0.24 & 6.23 & 50 & $\hat{x}$ & 85 & $\hat{x}$ & Herbig (1975) & 61.8 \\
\hline 166937 & 0.26 & 6.23 & $11.3 \pm 0.6$ & $\sqrt{ }$ & $50.5 \pm 0.8$ & $\hat{v}$ & Megier et al. (2005) & \\
\hline 167971 & 1.08 & 5.46 & 87 & 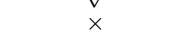 & 152 & $\mathrm{v}$ & Herbig (1975) & 124.0 \\
\hline 167971 & 1.08 & 5.46 & $39 \pm 2$ & $\sqrt{ }$ & $85 \pm 2$ & $\hat{v}$ & Thorburn et al. (2003) & \\
\hline 167971 & 1.05 & 5.46 & - & $x$ & $81.8 \pm 3.0$ & $\begin{array}{c}v \\
x\end{array}$ & Megier et al. (2005) & \\
\hline 168076 & 0.78 & 4.81 & $43 \pm 3$ & $\hat{v}$ & $108 \pm 4$ & $\hat{v}$ & Thorburn et al. (2003) & 151.0 \\
\hline 183143 & 1.28 & 6.13 & - & $x$ & 116.5 & $x$ & Cox et al. (2007) & 169.4 \\
\hline 183143 & 1.28 & 6.13 & - & $\hat{x}$ & $116.5 \pm 6.4$ & $\hat{x}$ & Sonnentrucher et al. (1997) & \\
\hline 183143 & 1.27 & 6.13 & - & $\hat{x}$ & $1854 \pm 60$ & $\hat{x}$ & Snow et al. (2002) & \\
\hline 183143 & 1.27 & 6.13 & $58 \pm 2$ & $\sqrt{ }$ & $113 \pm 3$ & $\hat{v}$ & Thorburn et al. (2003) & \\
\hline 183143 & 1.27 & 6.13 & 52 & $\sqrt{ }$ & 116 & $\sqrt{ }$ & Wszolk \& Godlowski (2003) & \\
\hline
\end{tabular}


Table 10-Continued

\begin{tabular}{|c|c|c|c|c|c|c|c|c|}
\hline $\mathrm{HD} / \mathrm{BD}$ & $\begin{array}{c}E(B-V) \\
(\mathrm{mag})\end{array}$ & $\begin{array}{c}W_{2175} / E(B-V) \\
\left(\mu \mathrm{m}^{-1}\right)\end{array}$ & $\begin{array}{c}W_{6376} \\
(\mathrm{~m} \AA)\end{array}$ & $\begin{array}{c}\text { Adopt " } \sqrt{ } \text { " } \\
\text { or Reject "X" }\end{array}$ & $\begin{array}{c}W_{6379} \\
(\mathrm{~m} \AA)\end{array}$ & $\begin{array}{c}\text { Adopt " } \sqrt{ } \text { " } \\
\text { or Reject "X" }\end{array}$ & Sources & $\begin{array}{c}W_{6376 / 6379}(\mathrm{~m} \AA) \\
\text { This Work } \\
\end{array}$ \\
\hline 183143 & 1.27 & 6.13 & $63.7 \pm 1.0$ & $\sqrt{ }$ & $105.4 \pm 0.7$ & $\sqrt{ }$ & Hobbs et al. (2009) & \\
\hline 185418 & 0.50 & 6.67 & $29 \pm 2$ & $\sqrt{ }$ & $71 \pm 2$ & $\sqrt{ }$ & Thorburn et al. (2003) & 100.0 \\
\hline 190603 & 0.71 & 4.88 & $24.6 \pm 3.2$ & $\sqrt{ }$ & $39.4 \pm 5.8$ & $\sqrt{ }$ & Megier et al. (2005) & 64.0 \\
\hline 190603 & 0.71 & 4.88 & 118 & $\times$ & 228 & $\times$ & Herbig (1975) & \\
\hline 190603 & 0.72 & 4.88 & - & $\times$ & $87.8 \pm 3.6$ & $\times$ & Sonnentrucher et al. (1997) & \\
\hline 197770 & 0.58 & 6.82 & - & $\times$ & 62.1 & $\times$ & Cox et al. (2007) & \\
\hline 198478 & 0.54 & 5.44 & - & $\times$ & $99.9 \pm 5.9$ & $\times$ & Sonnentrucher et al. (1997) & 131.3 \\
\hline 198478 & 0.54 & 5.44 & - & $\times$ & 82.1 & $\times$ & Cox et al. (2007) & \\
\hline 198478 & 0.54 & 5.44 & $21.1 \pm 1.6$ & $\times$ & $46.2 \pm 1.5$ & $\times$ & Megier et al. (2005) & \\
\hline 198478 & 0.54 & 5.44 & $22.6 \pm 1.5$ & $\sqrt{ }$ & $102 \pm 1.5$ & $\sqrt{ }$ & Thorburn et al. (2003) & \\
\hline 198478 & 0.53 & 5.44 & 25 & $\sqrt{ }$ & 113 & $\sqrt{ }$ & Herbig (1975) & \\
\hline 199478 & 0.47 & 6.42 & 25 & $\sqrt{ }$ & 85 & $\sqrt{ }$ & Herbig (1975) & 110.0 \\
\hline 199579 & 0.37 & 4.60 & $14.5 \pm 1$ & $\sqrt{ }$ & $19 \pm 2$ & $\sqrt{ }$ & Thorburn et al. (2003) & 32.7 \\
\hline 199579 & 0.36 & 4.60 & 40 & $\times$ & - & $\times$ & Herbig (1975) & \\
\hline 199579 & 0.35 & 4.60 & $14.3 \pm 2.0$ & $\sqrt{ }$ & $17.6 \pm 1.4$ & $\sqrt{ }$ & Megier et al. (2005) & \\
\hline 203938 & 0.74 & 5.70 & $29 \pm 2$ & $\sqrt{ }$ & $54 \pm 1$ & $\sqrt{ }$ & Thorburn et al. (2003) & 87.5 \\
\hline 203938 & 0.70 & 5.70 & $44.9 \pm 3.8$ & $\sqrt{ }$ & $47.0 \pm 2.0$ & $\sqrt{ }$ & Megier et al. (2005) & \\
\hline 204827 & 1.11 & 4.25 & $45 \pm 2$ & $\sqrt{ }$ & $96 \pm 1.5$ & $\sqrt{ }$ & Thorburn et al. (2003) & 140.3 \\
\hline 204827 & 1.11 & 4.25 & $44.6 \pm 1.1$ & $\sqrt{ }$ & $94.9 \pm 0.8$ & $\sqrt{ }$ & Hobbs et al. (2008) & \\
\hline 206165 & 0.46 & 6.07 & $18.9 \pm 0.8$ & $\sqrt{ }$ & $66.2 \pm 0.9$ & $\sqrt{ }$ & Megier et al. (2005) & 90.7 \\
\hline 206165 & 0.47 & 6.07 & - & $x$ & $61.1 \pm 13.6$ & $\times$ & Sonnentrucher et al. (1997) & \\
\hline 206165 & 0.47 & 6.07 & $23.2 \pm 1.5$ & $\sqrt{ }$ & $71.8 \pm 2$ & $\sqrt{ }$ & Thorburn et al. (2003) & \\
\hline 206165 & 0.47 & 6.07 & 21 & $\sqrt{ }$ & 71 & $\sqrt{ }$ & Wszolk \& Godlowski (2003) & \\
\hline 206267 & 0.53 & 4.96 & $23 \pm 2$ & $\sqrt{ }$ & $40 \pm 2$ & $\sqrt{ }$ & Thorburn et al. (2003) & 61.9 \\
\hline 206267 & 0.50 & 4.96 & $25.5 \pm 1.5$ & $\sqrt{ }$ & $36.3 \pm 0.9$ & $\sqrt{ }$ & Megier et al. (2005) & \\
\hline 206267 & 0.53 & 4.96 & 24 & $\sqrt{ }$ & 37 & $\sqrt{ }$ & Wszolk \& Godlowski (2003) & \\
\hline 207198 & 0.54 & 5.23 & $35.2 \pm 1.3$ & $\sqrt{ }$ & $73.7 \pm 0.8$ & $\sqrt{ }$ & Megier et al. (2005) & 109.6 \\
\hline 207198 & 0.62 & 5.23 & $35 \pm 2$ & $\sqrt{ }$ & $75 \pm 2$ & $\sqrt{ }$ & Thorburn et al. (2003) & \\
\hline 207198 & 0.62 & 5.23 & 30 & $\sqrt{ }$ & 80 & $\sqrt{ }$ & Wszolk \& Godlowski (2003) & \\
\hline 210121 & 0.40 & 2.74 & $3.9 \pm 1$ & $\sqrt{ }$ & $15.5 \pm 1$ & $\sqrt{ }$ & Thorburn et al. (2003) & 19.4 \\
\hline+631964 & 0.96 & 5.68 & $75.8 \pm 3.6$ & $\sqrt{ }$ & $168.0 \pm 2.6$ & $\sqrt{ }$ & Megier et al. (2005) & 246.9 \\
\hline+631964 & 1.00 & 5.68 & $75 \pm 3$ & $\sqrt{ }$ & $175 \pm 3$ & $\sqrt{ }$ & Thorburn et al. (2003) & \\
\hline
\end{tabular}


Table 11. Same as Table 3 but for the $\lambda 6614 \AA$ DIB.

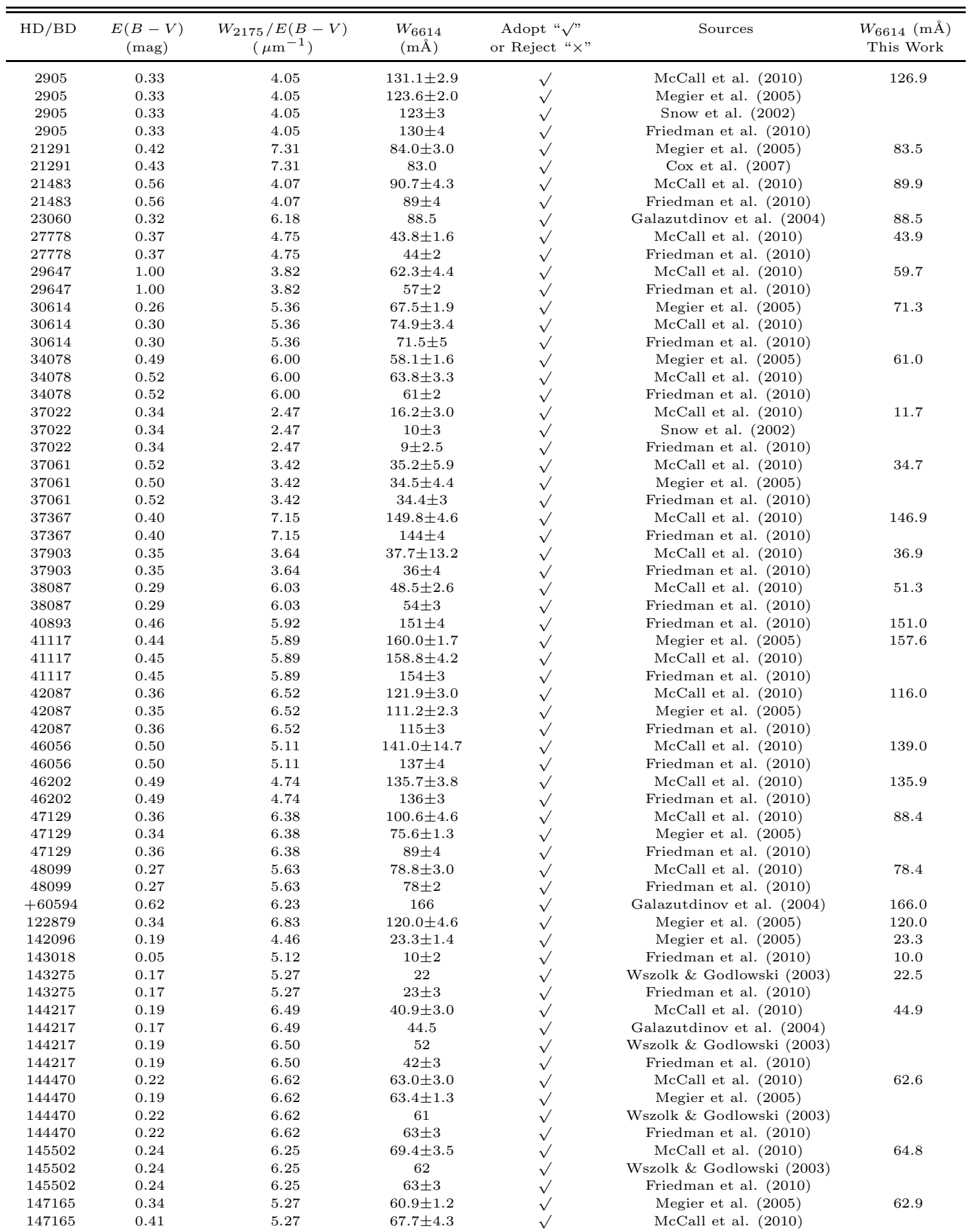


Table 11-Continued

\begin{tabular}{|c|c|c|c|c|c|c|}
\hline $\mathrm{HD} / \mathrm{BD}$ & $\begin{array}{c}E(B-V) \\
\quad(\mathrm{mag})\end{array}$ & $\begin{array}{c}W_{2175} / E(B-V) \\
\left(\mu \mathrm{m}^{-1}\right)\end{array}$ & $\begin{array}{c}W_{6614} \\
(\mathrm{~m} \AA)\end{array}$ & $\begin{array}{l}\text { Adopt " } \sqrt{ } \text { " } \\
\text { or Reject "X" }\end{array}$ & Sources & $\begin{array}{c}W_{6614}(\mathrm{~m} \AA) \\
\text { This Work }\end{array}$ \\
\hline 147165 & 0.41 & 5.27 & 60 & $\sqrt{ }$ & Wszolk \& Godlowski (2003) & \\
\hline 147888 & 0.47 & 5.87 & $78.5 \pm 2.2$ & $\sqrt{ }$ & McCall et al. (2010) & 80.8 \\
\hline 147888 & 0.47 & 5.87 & $82 \pm 4$ & $\sqrt{ }$ & Snow et al. (2002) & \\
\hline 147888 & 0.47 & 5.87 & $82 \pm 2$ & $\sqrt{ }$ & Friedman et al. (2010) & \\
\hline 147933 & 0.48 & 5.62 & $70.7 \pm 4.2$ & $\sqrt{ }$ & McCall et al. (2010) & \\
\hline 147933 & 0.48 & 5.62 & $68 \pm 5$ & $\sqrt{ }$ & Friedman et al. (2010) & \\
\hline 149757 & 0.29 & 6.63 & $40.5 \pm 2.0$ & $\sqrt{ }$ & Megier et al. (2005) & 40.8 \\
\hline 149757 & 0.32 & 6.63 & $38.2 \pm 1.9$ & $\sqrt{ }$ & McCall et al. (2010) & \\
\hline 149757 & 0.29 & 6.63 & 40.5 & $\sqrt{ }$ & Galazutdinov et al. (2004) & \\
\hline 149757 & 0.32 & 6.63 & 44 & $\sqrt{ }$ & Wszolk \& Godlowski (2003) & \\
\hline 166937 & 0.25 & 6.23 & $89.5 \pm 3.9$ & $\sqrt{ }$ & McCall et al. (2010) & 92.8 \\
\hline 166937 & 0.26 & 6.23 & $98.8 \pm 1.7$ & $\sqrt{ }$ & Megier et al. (2005) & \\
\hline 166937 & 0.25 & 6.23 & $90 \pm 3$ & $\sqrt{ }$ & Friedman et al. (2010) & \\
\hline 167971 & 1.08 & 5.46 & $220.7 \pm 6.0$ & $\sqrt{ }$ & McCall et al. (2010) & 224.2 \\
\hline 167971 & 1.05 & 5.46 & $233.0 \pm 6.8$ & $\sqrt{ }$ & Megier et al. (2005) & \\
\hline 167971 & 1.08 & 5.46 & $219 \pm 3$ & $\sqrt{ }$ & Friedman et al. (2010) & \\
\hline 168076 & 0.78 & 4.81 & $234.5 \pm 8.1$ & $\sqrt{ }$ & McCall et al. (2010) & 227.8 \\
\hline 168076 & 0.78 & 4.81 & $221 \pm 6$ & $\sqrt{ }$ & Friedman et al. (2010) & \\
\hline 183143 & 1.27 & 6.13 & $338.2 \pm 3.6$ & $\sqrt{ }$ & McCall et al. (2010) & 344.1 \\
\hline 183143 & 1.27 & 6.13 & $334 \pm 6$ & $\sqrt{ }$ & Snow et al. (2002) & \\
\hline 183143 & 1.28 & 6.13 & 368.6 & $\sqrt{ }$ & Cox et al. (2007) & \\
\hline 183143 & 1.27 & 6.13 & 350 & $\sqrt{ }$ & Wszolk \& Godlowski (2003) & \\
\hline 183143 & 1.27 & 6.13 & $332 \pm 4$ & $\sqrt{ }$ & Friedman et al. (2010) & \\
\hline 199579 & 0.35 & 4.60 & $47.7 \pm 2.1$ & $\sqrt{ }$ & Megier et al. (2005) & 56.7 \\
\hline 199579 & 0.37 & 4.60 & $59.5 \pm 3.7$ & $\sqrt{ }$ & McCall et al. (2010) & \\
\hline 199579 & 0.37 & 4.60 & $63 \pm 2$ & $\sqrt{ }$ & Friedman et al. (2010) & \\
\hline 203938 & 0.74 & 5.70 & $147.3 \pm 6.0$ & $\sqrt{ }$ & McCall et al. (2010) & 138.8 \\
\hline 203938 & 0.70 & 5.70 & $131.0 \pm 4.5$ & $\sqrt{ }$ & Megier et al. (2005) & \\
\hline 203938 & 0.66 & 5.70 & 131 & $\sqrt{ }$ & Galazutdinov et al. (2004) & \\
\hline 203938 & 0.74 & 5.70 & $146 \pm 3$ & $\sqrt{ }$ & Friedman et al. (2010) & \\
\hline 204827 & 1.06 & 4.25 & 161 & $\sqrt{ }$ & Galazutdinov et al. (2004) & 168.0 \\
\hline 204827 & 1.11 & 4.25 & $174.7 \pm 15.4$ & $\sqrt{ }$ & McCall et al. (2010) & \\
\hline 204827 & 1.11 & 4.25 & $171 \pm 3$ & $\sqrt{ }$ & Friedman et al. (2010) & \\
\hline 204827 & 1.11 & 4.25 & $165.1 \pm 1.4$ & $\sqrt{ }$ & Hobbs et al. (2008) & \\
\hline 206165 & 0.47 & 6.07 & $119.5 \pm 3.9$ & $\sqrt{ }$ & McCall et al. (2010) & 111.3 \\
\hline 206165 & 0.46 & 6.07 & $108.8 \pm 1.4$ & $\sqrt{ }$ & Megier et al. (2005) & \\
\hline 206165 & 0.47 & 6.07 & 106 & $\sqrt{ }$ & Wszolk \& Godlowski (2003) & \\
\hline 206165 & 0.47 & 6.07 & $111 \pm 3$ & $\sqrt{ }$ & Friedman et al. (2010) & \\
\hline 206267 & 0.53 & 4.96 & $120.9 \pm 4.3$ & $\sqrt{ }$ & McCall et al. (2010) & 120.0 \\
\hline 206267 & 0.50 & 4.96 & $117.0 \pm 1.1$ & $\sqrt{ }$ & Megier et al. (2005) & \\
\hline 206267 & 0.49 & 4.96 & 117 & $\sqrt{ }$ & Galazutdinov et al. (2004) & \\
\hline 206267 & 0.53 & 4.96 & 119 & $\sqrt{ }$ & Wszolk \& Godlowski (2003) & \\
\hline 206267 & 0.53 & 4.96 & $126 \pm 3$ & $\sqrt{ }$ & Friedman et al. (2010) & \\
\hline 207198 & 0.54 & 5.23 & $119.0 \pm 2.5$ & $\sqrt{ }$ & Megier et al. (2005) & 123.8 \\
\hline 207198 & 0.56 & 5.23 & 119 & $\sqrt{ }$ & Galazutdinov et al. (2004) & \\
\hline 207198 & 0.62 & 5.23 & $133.8 \pm 5.1$ & $\sqrt{ }$ & McCall et al. (2010) & \\
\hline 207198 & 0.62 & 5.23 & 122 & $\sqrt{ }$ & Wszolk \& Godlowski (2003) & \\
\hline 207198 & 0.62 & 5.23 & $125 \pm 3$ & $\sqrt{ }$ & Friedman et al. (2010) & \\
\hline 210121 & 0.40 & 2.74 & $28.4 \pm 12.2$ & $\sqrt{ }$ & McCall et al. (2010) & 26.7 \\
\hline
\end{tabular}


Table 11-Continued

\begin{tabular}{|c|c|c|c|c|c|c|}
\hline $\mathrm{HD} / \mathrm{BD}$ & $\begin{array}{c}E(B-V) \\
\quad(\mathrm{mag})\end{array}$ & $\begin{array}{c}W_{2175} / E(B-V) \\
\left(\mu \mathrm{m}^{-1}\right)\end{array}$ & $\begin{array}{c}W_{6614} \\
(\mathrm{~m} \AA)\end{array}$ & $\begin{array}{c}\text { Adopt " } " \\
\text { or Reject "X" }\end{array}$ & Sources & $\begin{array}{c}W_{6614}(\mathrm{~m} \AA) \\
\text { This Work }\end{array}$ \\
\hline 210121 & 0.40 & 2.74 & $25 \pm 2$ & $\sqrt{ }$ & Friedman et al. (2010) & \\
\hline 217086 & 0.92 & 6.48 & 215 & $\sqrt{ }$ & Galazutdinov et al. (2004) & 215.0 \\
\hline+631964 & 1.00 & 5.68 & $334.0 \pm 5.8$ & $\sqrt{ }$ & McCall et al. (2010) & 325.9 \\
\hline+631964 & 0.96 & 5.68 & $313.8 \pm 6.0$ & $\sqrt{ }$ & Megier et al. (2005) & \\
\hline+631964 & 1.00 & 5.68 & $330 \pm 6$ & $\sqrt{ }$ & Friedman et al. (2010) & \\
\hline
\end{tabular}

